Maladies chroniques

et blessures au Canada

une publication de l'Agence de la santé publique du Canada

Comité de rédaction de $\mathrm{MCBC}$

Howard Morrison, Ph. D. Rédacteur scientifique en chef (613) 941-1286

Robert A. Spasoff, M.D. Rédacteur scientifique adjoint

Claire Infante-Rivard, M.D.

Rédactrice scientifique adjointe

Elizabeth Kristjansson, Ph. D.

Rédactrice scientifique adjointe

Michelle Tracy, M.A. Gestionnaire de la rédaction
Lesley Doering, M.T.S.

Agence de la santé publique du Canada

Robert Geneau, Ph. D.

Agence de la santé publique du Canada

Isra Levy, M.B., FRCPC, FACPM

Santé publique Ottawa

Lesli Mitchell, M.A.

Centers for Disease Control and Prevention

Scott Patten, M.D., Ph. D., FRCPC University of Calgary

Barry Pless, C.M., M.D., FRCPC Hôpital de Montréal pour enfants

Kerry Robinson, Ph. D. Agence de la santé publique du Canada

Fabiola Tatone-Tokuda, M. Sc. Université d'Ottawa

Andreas T. Wielgosz, M.D., Ph. D., FRCPC Agence de la santé publique du Canada

Don Wigle, M.D., Ph. D. Université d'Ottawa

Russell Wilkins, M. Urb. Statistique Canada
Maladies chroniques et blessures au Canada (MCBC) est une revue scientifique trimestrielle mettant au point les données probantes actuelles sur la prévention et la lutte contre les maladies chroniques (c.-à-d. non transmissibles) et les traumatismes au Canada. Selon une formule unique et depuis 1980 , la revue publie des articles soumis à l'examen par les pairs des auteurs et provenant des secteurs tant public que privé et comprenant des recherches effectuées dans des domaines tels que l'épidémiologie, la santé publique ou communautaire, la biostatistique, les sciences du comportement, et l'économie ou les services de la santé. Tous les articles de fond sont soumis à l'examen par les pairs des auteurs; les autres types d'articles ne le sont pas. Les auteurs demeurent responsables du contenu de leurs articles, et les opinions exprimées ne sont pas forcément celles du Comité de rédaction de $\mathrm{MCBC}$, ni celles de l'Agence de la santé publique du Canada.

Maladies chroniques et blessures au Canada

Agence de la santé publique du Canada 785, avenue Carling, Indice de l'adresse : 6805B

Ottawa (Ontario) K1A 0K9

Télécopieur : (613) 941-9502

Courriel : cdic-mcbc@phac-aspc.gc.ca

Indexée dans Index Medicus/MEDLINE SciSearch ${ }^{\circledR}$ et Journal Citation Reports/ Science Edition

Promouvoir et protéger la santé des Canadiens grâce au leadership, aux partenariats, à l'innovation et aux interventions en matière de santé publique - Agence de la santé publique du Canada

Publication autorisée par le ministre de la Santé. (c) Sa Majesté la Reine du Chef du Canada, représentée par le ministre de la Santé, 2011 ISSN 1925-6531

On peut consulter cette publication par voie électronique dans le site Web www.santepublique.gc.ca/mcbc Also available in English under the title: Chronic Diseases and Injuries in Canada 


\section{Table des matières}

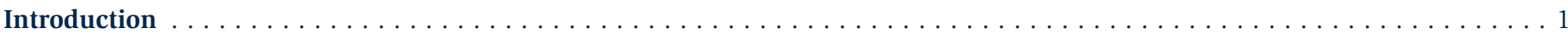

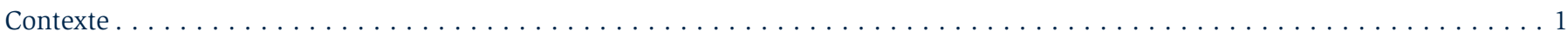

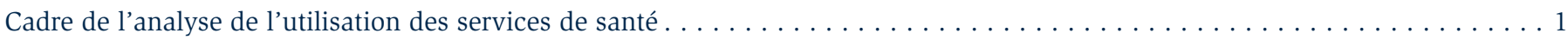

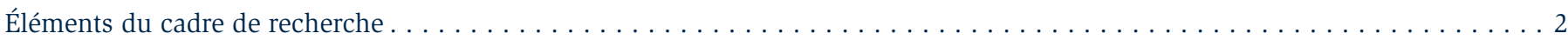

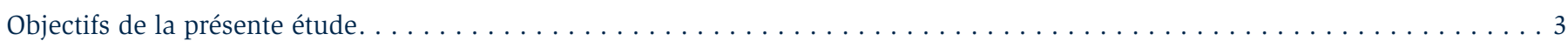

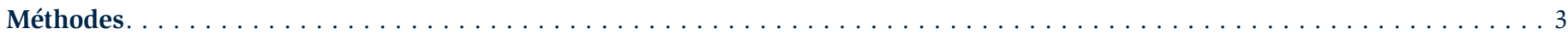

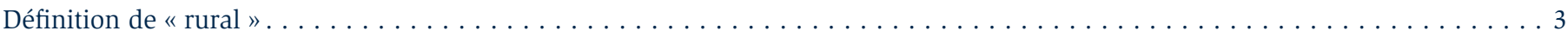

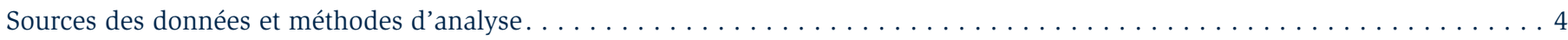

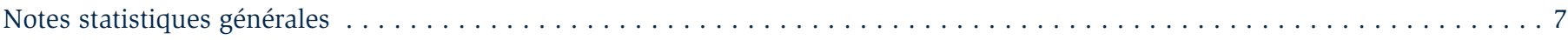

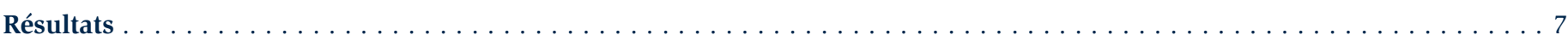

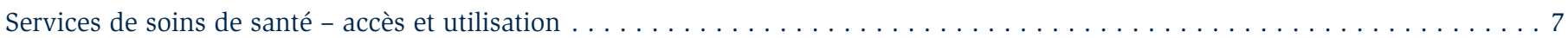

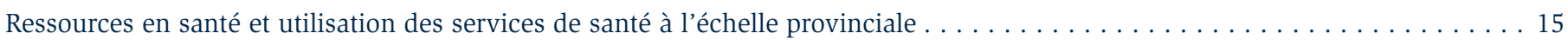

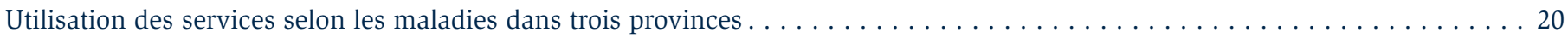

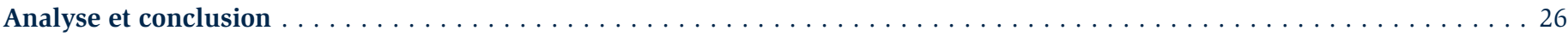

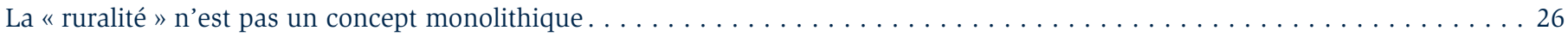

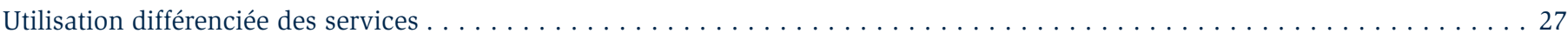

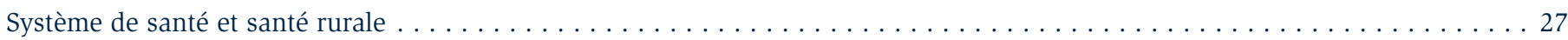

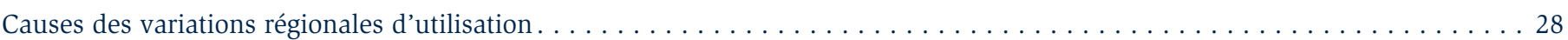

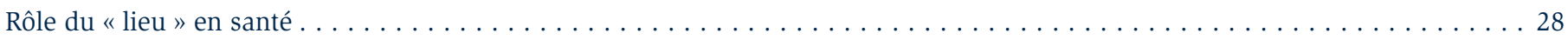

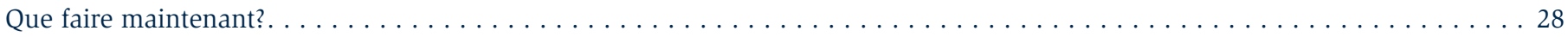

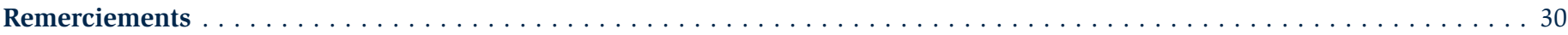

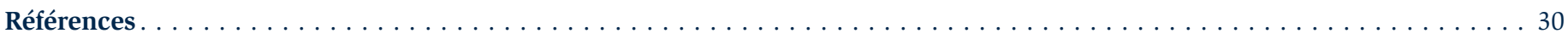

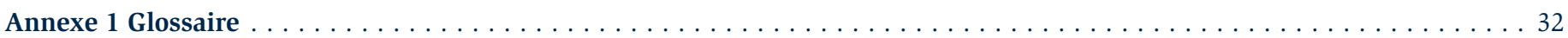

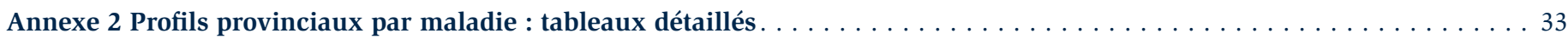




\section{Liste des tableaux}

Tableau 1. Population canadienne selon le degré de ruralité, 1996 et $2001 \ldots \ldots \ldots$

Tableau 2. Groupes de spécialités médicales utilisés dans l’analyse des demandes de paiement des médecins . . . . . . . . . 5

Tableau 3. Diagnostics utilisés dans la comparaison des risques relatifs des visites chez le médecin et des hospitalisations,

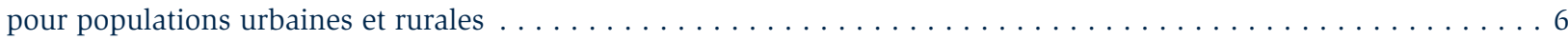

Tableau 4. Nombre de médecins et ratios de médecins pour 10000 habitants,

selon le type de médecin et le lieu de résidence, $2001 \ldots \ldots \ldots \ldots \ldots$

Tableau 5. Proportion (normalisée selon l'âge) de Canadiens de 12 ans et plus qui ont déclaré avoir consulté un médecin au cours des 12 derniers mois, selon le lieu de résidence, 2000-2001 . . . . . . . . . . . . . . 12

Tableau 6. Proportion (normalisée selon l'âge) des personnes de 15 ans et plus qui ont déclaré avoir consulté un dentiste, une infirmière ou d'autres fournisseurs de soins de santé, selon le lieu de résidence et le sexe, Canada, 2000-2001 . . . . 12

Tableau 7. Taux de congé d'hôpital (normalisés selon l'âge) pour 1000 habitants, selon le lieu de résidence

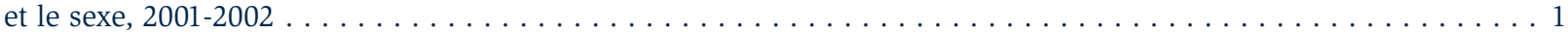

Tableau 8. Durée moyenne d'hospitalisation, en jours, selon le groupe d’âge, le sexe et le lieu de résidence,

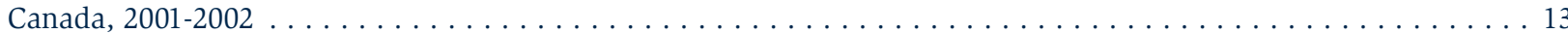

Tableau 9. Rapports de cotes corrigés établissant le lien entre le lieu de résidence et l'hospitalisation au cours

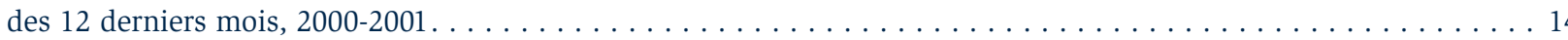

Tableau 10. Proportion (normalisée selon l'âge) de Canadiens de 15 ans et plus qui ont donné leur opinion sur la qualité des soins de santé reçus au cours des 12 derniers mois et leur niveau de satisfaction à leur égard, selon le lieu

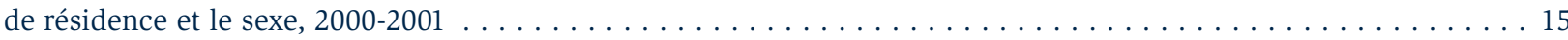

Tableau 11. Population selon le degré de ruralité, Nouvelle-Écosse, Ontario et Colombie-Britannique, 2001 . . . . . . . . . . . . 16

Tableau 12. Sommaire des risques relatifs de consultation d'un médecin et d'hospitalisation pour certaines catégories de maladies, selon le lieu de résidence et le sexe, Nouvelle-Écosse, 2001-2002 . . . . . . . . . . . . . 21

Tableau 13. Risques relatifs de consultation chez le médecin (normalisée selon l'âge) pour troubles mentaux, traumatismes ou intoxications, selon le lieu de résidence et le sexe, Nouvelle-Écosse, 2001-2002 . . . . . . . . . . . 22

Tableau 14. Risques relatifs de congé d'hôpital (normalisé selon l’âge) pour troubles mentaux, selon le lieu de résidence et le sexe, Nouvelle-Écosse, $2001-2002 \ldots \ldots \ldots \ldots \ldots$. . . . . . . . . . . . . . 22

Tableau 15. Risques relatifs de consultation d'un médecin et d'hospitalisation pour certaines catégories de maladies,

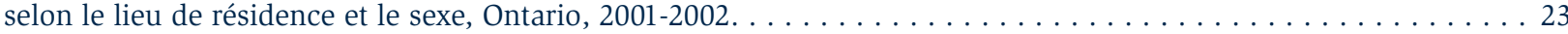

Tableau 16. Risques relatifs de consultation d'un médecin (normalisée selon l'âge) pour traumatismes et intoxications, selon le lieu de résidence et le sexe, Ontario, 2001-2002.

Tableau 17. Risques relatifs de congé d'hôpital (normalisé selon l’âge) pour troubles mentaux, selon le lieu de résidence et le sexe, Ontario, $2001-2002 \ldots \ldots \ldots \ldots \ldots$

Tableau 18. Risques relatifs de consultation d'un médecin et d'hospitalisation pour certaines maladies, selon le lieu de résidence et le sexe, Colombie-Britannique, 2001-2002 . 


\section{Liste des figures}

Figure 1. Adaptation du nouveau modèle comportemental à l'utilisation des services de santé par la population . . . . . . . . . . 1

Figure 2. Proportion (normalisée selon l’âge) de Canadiens de 15 ans et plus qui ont eu besoin de soins de routine

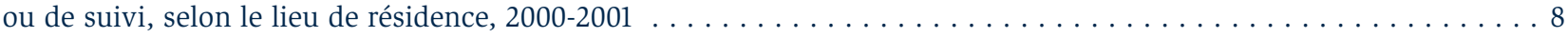

Figure 3. Proportion (normalisée selon l'âge) de Canadiens de 15 ans et plus ayant eu besoin de soins immédiats pour un problème de santé mineur au cours de l'année précédente, selon le lieu de résidence, 2000-2001 . . . . . . . . . 9

Figure 4. Proportion (normalisée selon l'âge) de Canadiens de 15 ans et plus ayant déclaré avoir attendu plusieurs mois avant d'obtenir des soins spécialisés ou de subir une chirurgie non urgente, selon le lieu de résidence, 2000-2001 . . . 9

Figure 5. Proportion (normalisée selon l'âge) des hommes de 12 ans et plus ayant déclaré avoir consulté un médecin au cours des 12 derniers mois, selon le type de médecin et le lieu de résidence, Canada, 2000-2001 . . . . . . . . . . 10

Figure 6. Proportion (normalisée selon l’âge) des femmes de 12 ans et plus ayant déclaré avoir consulté un médecin au cours des 12 derniers mois, selon le type de médecin et le lieu de résidence, Canada, 2000-2001 . . . . . . . . . . .11

Figure 7. Taux annuels moyens de consultation des médecins de famille (normalisée selon l'âge) pour 1000 habitants, selon le lieu de résidence et le sexe, Nouvelle-Écosse, périodes de deux ans, 1997-2002 . . . . . . . . . . . . . 16

Figure 8. Taux annuels moyens de consultation des médecins spécialistes (normalisée selon l'âge) pour 1000 habitants, selon le lieu de résidence et le sexe, Nouvelle-Écosse, périodes de deux ans, 1997-2002 . . . . . . . . . . . . . . . 17

Figure 9. Taux annuels moyens de consultation des chirurgiens (normalisée selon l'âge) pour 1000 habitants, selon le lieu de résidence et le sexe, Nouvelle-Écosse, périodes de deux ans, 1997-2002 . . . . . . . . . . . . . 17

Figure 10. Taux annuels moyens de consultation des médecins de famille (normalisée selon l'âge) pour 1000 habitants, selon le lieu de résidence et le sexe, Ontario, périodes de deux ans, 1997-2002 . . . . . . . . . . . . . . . . 18

Figure 11. Taux annuels moyens de consultation des médecins spécialistes (normalisée selon l'âge) pour 1000 habitants, selon le lieu de résidence et le sexe, Ontario, périodes de deux ans, 1997-2002 .

Figure 12. Taux annuels moyens de consultation des chirurgiens (normalisée selon l'âge) pour 1000 habitants, selon le lieu de résidence et le sexe, Ontario, périodes de deux ans, 1997-2002 . . . . . . . . . . . . . . . . . . . 19 


\section{Liste des tableaux des annexes}

Tableau A1. Risques relatifs de consultation chez le médecin (normalisée selon l'âge) pour maladies de la circulation, selon le lieu de résidence et le sexe, Ontario et Nouvelle-Écosse, 2001-2002, et Colombie-Britannique, 2000-2001 . . . 33

Tableau A2. Risques relatifs de congé d’hôpital (normalisé selon l’âge) pour maladies de la circulation, selon le lieu de résidence et le sexe, Ontario, Nouvelle-Écosse et Colombie-Britannique, 2001-2002 . . . . . . . . 33

Tableau A3. Risques relatifs de consultation chez le médecin (normalisée selon l'âge) pour cancers (néoplasmes), selon le lieu de résidence et le sexe, Ontario et Nouvelle-Écosse, 2001-2002, et Colombie-Britannique, 2000-2001 . . . 34

Tableau A4. Risques relatifs de congé d'hôpital (normalisé selon l'âge) pour cancers (néoplasmes), selon le lieu de résidence et le sexe, Ontario, Nouvelle-Écosse et Colombie-Britannique, 2001-2002 . . . . . . . . 34

Tableau A5. Risques relatifs de consultation chez le médecin (normalisée selon l'âge) pour maladies du système respiratoire, selon le lieu de résidence et le sexe, Ontario et Nouvelle-Écosse, 2001-2002, et Colombie-Britannique, 2000-2001 . . . 35

Tableau A6. Risques relatifs de congé d'hôpital (normalisé selon l'âge) pour maladies du système respiratoire, selon le lieu de résidence et le sexe, Ontario, Nouvelle-Écosse et Colombie-Britannique, 2001-2002

Tableau A7. Risques relatifs de consultation chez le médecin (normalisée selon l'âge) pour maladies de l'appareil locomoteur et du tissu conjonctif, selon le lieu de résidence et le sexe, Ontario et Nouvelle-Écosse, 2001-2002,

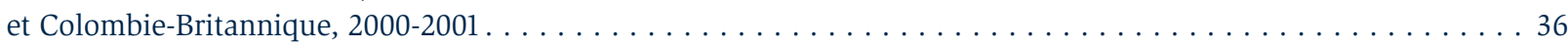

Tableau A8. Risques relatifs de congé d'hôpital (normalisé selon l'âge) pour maladies de l'appareil locomoteur et du tissu conjonctif, selon le lieu de résidence et le sexe, Ontario, Nouvelle-Écosse et Colombie-Britannique, 2001-2002 . . . . 36

Tableau A9. Risques relatifs de consultation chez le médecin (normalisée selon l'âge) pour traumatisme ou intoxication, selon le lieu de résidence et le sexe, Ontario et Nouvelle-Écosse, $2001-2002 \ldots \ldots \ldots$. . . . . . . . . . 37

Tableau A10. Risques relatifs de congé d'hôpital (normalisé selon l'âge) pour traumatisme ou intoxication, selon le lieu de résidence et le sexe, Ontario, Nouvelle-Écosse et Colombie-Britannique, 2001-2002 . . . . . . . . . 37

Tableau A11. Risques relatifs de consultation chez le médecin (normalisée selon l'âge) pour troubles mentaux, selon le lieu de résidence et le sexe, Ontario et Nouvelle-Écosse, 2001-2002 et Colombie-Britannique, $2000-2001$. . 37

Tableau A12. Risques relatifs de congé d’hôpital (normalisé selon l'âge) pour troubles mentaux, selon le lieu de résidence et le sexe, Ontario, Nouvelle-Écosse et Colombie-Britannique, 2001-2002 . . . . . . . . 38

Tableau A13. Risques relatifs de consultation chez le médecin (normalisée selon l'âge) pour maladies du système nerveux ou des organes sensoriels, selon le lieu de résidence et le sexe, Ontario et Nouvelle-Écosse, 2001-2002

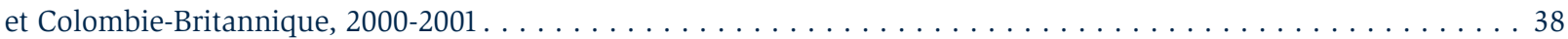

Tableau A14. Risques relatifs de congé d'hôpital (normalisé selon l'âge) pour maladies du système nerveux ou des organes sensoriels, selon le lieu de résidence et le sexe, Ontario, Nouvelle-Écosse et Colombie-Britannique, 2001-2002.

Tableau A15. Risques relatifs de consultation chez le médecin (normalisée selon l'âge) pour diabète, selon le lieu de résidence et le sexe, Ontario et Nouvelle-Écosse, 2001-2002, et Colombie-Britannique, 2000-2001 . . . 39 


\title{
'utilisation des services de santé dans les régions rurales du Canada
}

\author{
R.W. Pong, Ph. D. (1); M. DesMeules, M. Sc. (2); D. Heng, M. Sc. (1); C. Lagacé, M. Sc. (2) J. R. Guernsey, M. Sc., Ph. D. (3); \\ A. Kazanjian, D. Soc. (4); D. Manuel, M. D., M. Sc. (5); J. R. Pitblado, Ph. D. (1); R. Bollman, Ph. D. (6); I. Koren, M. Sc. (1); \\ M.P. Dressler, M. Sc. (2); F. Wang, M. Sc. (2); W. Luo, M. Sc. (2)
}

\section{Introduction}

\section{Contexte}

Les Canadiens ont à cœur la facilité d'accès aux services de santé. Bien que de nombreuses études aient porté sur l'accessibilité des soins de santé au Canada, très peu d'entre elles l'ont examinée sous l'angle des différences entre les régions urbaines et rurales, particulièrement dans une perspective à échelle nationale. Pourtant, il existe des disparités entre les populations des régions urbaines et rurales, tout comme il existe des différences entre les services dans les régions éloignées et dans les régions à faible densité de population.

Les collectivités rurales au Canada : comprendre la santé rurale et ses déterminants est un programme de recherche de trois ans financé par l'Initiative sur la santé de la population canadienne (ISPC) de l'Institut canadien d'information sur la santé (ICIS) et par l'Agence de la santé publique du Canada (l'ASPC). Y participent entre autres des chercheurs de l'Agence de la santé publique du Canada (ASPC) et du Centre de recherche en santé dans les milieux ruraux et du Nord de l'Université Laurentienne et d'autres chercheurs. Le premier document publié dans le cadre de ce programme de recherche s'intitule Comment se portent les Canadiens vivant en milieu rural? Une évaluation de leur état de santé et des déterminants de la santé ${ }^{1}$. Le présent document constitue la seconde publication de ce programme : il s'agit d'une analyse descriptive et comparative de l'utilisation d'un grand nombre de services de santé par les populations rurales et urbaines.

\section{Cadre de l'analyse de l'utilisation des services de santé}

L'endroit où nous vivons influe sur notre santé, par l'intermédiaire des conditions environnementales, climatiques et socioéconomiques, des activités professionnelles, de la composition ethnique, de la culture et des spécificités de la collectivité. Les caractéristiques des lieux et des personnes qui y vivent sont liées inextricablement et il faut les prendre en compte, ainsi que le système de soins de santé lui-même, lors de toute analyse portant sur la santé. Nous avons utilisé un modèle théorique conçu par Andersen ${ }^{2}$ pour orienter la recherche et l'analyse à la base de ce rapport. Ce "nouveau modèle comportemental » établit les facteurs prédisposants, les facteurs habilitants et les besoins qui influencent d'une manière

FIGURE 1

Adaptation du nouveau modèle comportemental à l'utilisation des services de santé par la population



Adapté avec la permission de R.M. Andersen².

Rattachement des auteurs

1 Centre de recherche en santé dans les milieux ruraux et du nord, Université Laurentienne

2 Agence de la santé publique du Canada

3 Université Dalhousie

4 Université de la Colombie-Britannique

5 Institut de recherche en services de santé

6 Statistique Canada

Correspondance : Raymond Pong, Centre for Rural and Northern Health Research, Laurentian University, 935 Ramsey Lake Road, Sudbury (Ontario) Canada P3E 2C6; tél. : 705-675-1151 poste 4357; téléc. : 705- 675-4855;

courriel : rpong@laurentienne.ca 
déterminante l'utilisation des services de santé (figure 1$)^{2}$. Parmi les facteurs prédisposants, il y a l'âge, le sexe et l'état civil; les facteurs habilitants comprennent, entre autres, les conditions externes qui facilitent ou freinent l'utilisation des services de santé (comme la distance à parcourir pour se rendre au cabinet du médecin); enfin, les besoins, tant du point de vue du patient que de celui du fournisseur de soins de santé, correspondent à l'état pathologique ou aux incapacités.

Le travail des chercheurs était de déterminer lesquels parmi ces facteurs sont importants dans une situation particulière et de quelle façon ils interagissent pour faciliter ou freiner l'utilisation des services de santé.

\section{Éléments du cadre de recherche}

\section{Environnement}

Le régime de soins de santé universel garantit à tous les Canadiens un accès aux soins médicaux et hospitaliers essentiels. Cependant, les collectivités éloignées ou peu peuplées ont à leur disposition proportionnellement moins de fournisseurs de services et, par conséquent, moins de services. Par exemple, en dépit du fait que $21,1 \%$ de la population canadienne vivait dans des régions rurales en 2004, seulement $9,4 \%$ de tous les médecins (16\% de médecins de famille et 2,4\% de médecins spécialistes) exerçaient dans ces régions ${ }^{3}$. Une étude des facteurs qui influencent l'aiguillage vers des médecins spécialistes de l'Ontario a révélé que le type de collectivité où exerce un médecin - région rurale, petite ville, collectivité où n'exercent que des médecins de premier recours, ou agglomération urbaine possédant ou non des hôpitaux universitaires - est le prédicteur le plus précis des taux d'aiguillage vers des médecins spécialistes, et que, des quatre types de collectivité, c'est dans les régions rurales que ce taux est le plus faible ${ }^{4}$.

L'environnement externe est composé de facteurs comme la situation économique, la richesse relative, la politique et les normes de la société. Par exemple, les collectivités rurales sont aux prises avec un certain nombre de difficultés sur les plans économique et de l'emploi. Les changements technologiques et l'épuisement des ressources peuvent limiter l'emploi dans certaines régions rurales, et la centralisation des services peut ébranler la viabilité économique et sociale de certaines autres. Les données factuelles donnent à penser que le chômage et l'incertitude économique sont une source de maladies physiques, de stress mental et de taux élevés de mortalité, conditions qui pourraient donner lieu à un besoin et à une utilisation plus intenses de services de santé5. En plus d'être exposés aux changements économiques, de nombreux services de santé dépendent de plus en plus de technologies sophistiquées et coûteuses que seuls les grands hôpitaux des grandes collectivités peuvent se permettre, ce qui pourrait se traduire par un manque de ressources et de services de santé dans les régions rurales.

\section{Facteurs prédisposants}

La conjoncture sociodémographique des collectivités rurales influe différemment de celle des collectivités urbaines sur l'utilisation des services de santé. Par exemple, de nombreuses collectivités rurales ont un rapport de dépendance plus élevé, car elles comptent une plus grande proportion d'enfants et de personnes âgées ${ }^{6}$; en outre, les très jeunes enfants et les vieillards font plus souvent appel aux services de santé que la population en âge de travailler*. Les caractéristiques d'une collectivité peuvent également avoir une grande influence. Une étude a révélé que chez les femmes, la perception de la qualité de la vie dans la collectivité est étroitement liée à la perception de leur état de santé et de leur santé fonctionnelle; les hommes, quant à eux, perçoivent leur environnement physique par rapport à leur état de santé et à leur santé fonctionnelle $e^{7}$. Il s'avère que les femmes des collectivités encourageant la mammographie recourent en plus grand nombre à ce mode de dépistage que celles des collectivités qui sont plus réfractaires à cette technique de prévention ${ }^{8}$.
Les convictions relatives à la santé sont un déterminant important de l'utilisation des services de santé. Par exemple, les Australiens des régions rurales considèrent la santé d'un point de vue négatif, c'est-à-dire comme une absence de maladie ${ }^{9}$ et par conséquent, ils pourraient attacher une plus grande importance à la guérison et à l'atténuation des symptômes ou de l'inconfort plutôt qu'à la prévention des maladies ou au maintien d'une bonne santé $^{10}$. Les valeurs traditionnelles en région rurale comme l'autonomie, l'indépendance et la préférence pour les réseaux de soutien informels peuvent aussi mener à une baisse de l'utilisation des services préventifs ou d'autres services de santé à moins qu'un problème grave de santé ne survienne.

\section{Facteurs habilitants}

Les problèmes de transport sont une des principales préoccupations des résidents des régions rurales lorsqu'il est question de l'accès aux services de santél1. D'ailleurs, les services de transport en commun ont été réduits ou sont devenus plus coûteux dans de nombreuses collectivités rurales du Canada. Certains programmes provinciaux ont toutefois vu le jour pour faciliter l'accès aux services de soins de santé. En Ontario par exemple, un programme d'extension des services médicaux et un programme de consultations offertes par des spécialistes itinérants ont été mis sur pied dans le cadre du Programme des services aux régions insuffisamment desservies. Dans d'autres provinces et territoires, les patients des régions rurales peuvent obtenir de l'aide pour se déplacer en vue d'obtenir des soins qui ne sont pas offerts près de chez eux. Les effets sont quantifiables : par exemple, le taux de dépistage par mammographie dans les régions rurales du Manitoba a augmenté grâce à l'utilisation d'unités de dépistage mobiles ${ }^{12,13}$.

Les résidents des régions rurales, pour protéger leur vie privée, peuvent également hésiter à se faire soigner si la petite taille de leur collectivité et les liens étroits entre habitants risquent de compromettre leur anonymat. La protection de la vie privée

\footnotetext{
* Dans notre étude, le rapport de dépendance plus élevé dans les régions rurales ne se manifestera peut-être pas par des taux plus élevés d'accès aux services de santé, car nos résultats sont normalisés ou rectifiés selon l'âge. L'âge est pris en compte dans les modèles d'analyse multivariée seulement pour établir qu'il est associé à l'utilisation des services.
} 
compte davantage pour les jeunes femmes des régions rurales que pour celles des régions urbaines dans la décision de consulter un médecin ${ }^{14}$.

\section{Besoins}

Les ressources consacrées aux soins de santé pour les Canadiens souffrant d'une maladie chronique couvrent $67 \%$ de l'ensemble des coûts directs (soins de santé) et $60 \%$ des coûts indirects (perte de productivité et de revenus) ${ }^{15}$. En général, les risques de mortalité attribuables aux maladies chroniques comme les maladies du système circulatoire et du système respiratoire ainsi que le diabète sont plus élevés dans les régions rurales que dans les régions urbaines ${ }^{1}$. D'autres études ont révélé qu'une proportion plus élevée de la population rurale ou nordique considère avoir un état de santé mauvais ou passable, des limitations d'activités et des incapacités ${ }^{16-18}$. Toutes choses étant égales par ailleurs, les personnes dont l'état de santé est mauvais ou dont les besoins sont plus grands consulteront le médecin ou utiliseront d'autres services de santé plus souvent.

\section{Utilisation des services de santé}

Peu de données nationales existent sur l'utilisation des services de santé par les Canadiens des régions rurales, malgré le fait que les provinces aient analysé leurs propres données sur les consultations médicales et les admissions dans les hôpitaux. Par exemple, en 2000, les taux de consultation du médecin, de recours aux unités de soins ambulatoires et de consultation de spécialistes itinérants chez les résidents des régions rurales et nordiques du Manitoba étaient moindres que les taux provinciaux moyens ${ }^{13}$. En Ontario, les taux de congé d'hôpital pour soins de courte durée des régions rurales étaient de près de $50 \%$ plus élevés que le taux moyen provincial ${ }^{17}$. Au Québec, le taux de congé s'est également avéré plus élevé dans les régions rurales, en dépit du fait que la durée de l'hospitalisation ait été plus courte que dans les régions urbaines ${ }^{16}$. En outre, les services d'urgence des hôpitaux ruraux reçoivent un plus grand nombre de patients qui ne nécessitent pas de soins urgents (c'est-à-dire dont les interventions peuvent être reportées) que ceux des hôpitaux en région urbaine ${ }^{19}$.

\section{Objectifs de la présente étude}

La présente étude, en s'appuyant sur ce cadre de recherche, tente d'évaluer les tendances nationales de l'utilisation des services de santé par les Canadiens des milieux ruraux et à les comparer à celles des citadins. Elle utilise les résultats d'une analyse descriptive et d'une analyse bidimensionnelle de mesures particulières de l'utilisation des services de santé, ainsi que des constatations découlant d'analyses de régression multivariées portant sur des facteurs connexes.

Nous avons établi les questions de recherche suivantes :

- En quoi l'utilisation dans les régions rurales des services médicaux et d'autres services de santé, y compris les services aux malades hospitalisés, diffère-t-elle de celle des régions urbaines au Canada?

- En plus des différences dans l'utilisation des services de santé entre les régions urbaines et rurales, existe-t-il des disparités entre les régions rurales?

- L'utilisation des services de santé par les Canadiens ruraux et urbains diffèret-elle selon le type de maladie?

- Quels sont les profils géographiques provinciaux de l'utilisation des services hospitaliers ou médicaux en Nouvelle-Écosse, en Ontario et en Colombie-Britannique?

- Le lieu de résidence est-il un déterminant de l'utilisation des services de santé?

\section{Méthodes}

\section{Définition de « rural »}

Il n'existe au Canada aucune définition unique et officielle des collectivités " rurales », et un certain nombre de définitions ont été adoptées pour diverses raisons. Dans le cadre de notre projet de recherche, nous avons utilisé la définition de Statistique Canada ${ }^{20}$ de " régions rurales et petites villes »(RRPV) dans l'analyse des données secondaires. La catégorie des RRPV désigne la population demeurant en dehors des zones de navettage des grands centres urbains, c'est-à-dire en dehors des régions métropolitaines de recensement (RMR) et des agglomérations de recensement (AR). Nous avons choisi cette définition en grande partie à cause de l'hétérogénéité des régions rurales, ce qui nous a permis de les subdiviser en quatre «zones d'influence métropolitaine» (ZIM) selon leur degré de ruralité. Cette définition tient également compte de la distance aux centres urbains, où nombre des services de santé spécialisés sont localisés.

Prolongement du concept de RRPV, la notion de ZIM a été élaborée par Statistique Canada " pour mieux montrer les effets de l'accessibilité métropolitaine sur les régions non métropolitaines $»^{20,21}$. Les taux de navettage des ZIM sont calculés à l'aide des données de recensement sur les lieux de travail. Cette méthode reconnaît la possibilité d'une " pluralité de centres d'attraction »: on combine les taux de navettage entre une collectivité de la catégorie RRPV et tout grand centre urbain de 10000 habitants ou plus pour établir le degré d'influence (forte, modérée, faible ou nulle) qu'exercent un ou plusieurs de ces centres sur la municipalité en question ${ }^{22}$. Cette classification et la méthodologie connexe ont été validées par Statistique Canada à de nombreuses reprises ${ }^{21}$.

Le classement par ZIM permet de distinguer les populations rurales qui ont un accès plus restreint au marché du travail des grands centres urbains de celles qui ont un accès plus facile. La distance entre les collectivités urbaines et rurales est un des principaux déterminants de l'accessibilité. Le taux de navettage de la population active sert à déterminer l'accessibilité à des services de santé et d'enseignement et à des services financiers, gouvernementaux, commerciaux, culturels et sportifs. Ce taux représente l'influence relative qu'exercent un ou plusieurs centres urbains sur une région rurale. Bien que les collectivités rurales comptent, par définition, moins de 10000 habitants, le classement par ZIM 
TABLEAU 1

Population canadienne selon le degré de ruralité, 1996 et 2001

\begin{tabular}{lrrrrr}
\hline & \multicolumn{3}{c}{$\begin{array}{c}\text { Effectif et répartition en pourcentage } \\
\text { (en fonction du découpage de 2001) }\end{array}$} & $\begin{array}{c}\text { Variation en } \\
\text { pourcentage parmi } \\
\text { les groupes de ZIM } \\
\text { entre 1996 et 2001 }\end{array}$ \\
\hline $\begin{array}{l}\text { Région urbaine } \\
\text { (RMR-AR) }\end{array}$ & 22654692 & 78,5 & 23839086 & 79,4 & $+5,2$ \\
$\begin{array}{l}\text { Ensemble } \\
\text { des RRPV }\end{array}$ & 6192069 & 21,5 & 6168008 & 20,6 & $-0,4$ \\
$\begin{array}{l}\text { ZIM forte } \\
\text { ZIM modérée }\end{array}$ & 1470493 & 5,1 & 1524579 & 5,1 & $+3,7$ \\
ZIM faible & 2307387 & 8,0 & 2285538 & 7,6 & $-0,9$ \\
Zone nulle & 2027488 & 7,0 & 1969211 & 6,6 & $-2,9$ \\
\hline Total & 386701 & 1,3 & 388680 & 1,3 & $+0,5$ \\
\hline
\end{tabular}

Source : Statistique Canada, Recensement de la population, 1996 et 2001.

Abréviations : AR, agglomération de recensement; RMR, région métropolitaine de recensement; ZIM, zone d'influence métropolitaine; RRPV, régions rurales et petites villes.

ne tient pas compte des différentes tailles des populations des divers types de collectivité rurale.

Notre étude est fondée sur les catégories géographiques suivantes et le tableau 1 illustre la répartition de la population canadienne selon ces catégories :

- Une région métropolitaine de recensement (RMR) est formée d'un noyau urbain d'au moins 100000 habitants et de toutes les villes et municipalités voisines dont $50 \%$ ou plus de la population active fait la navette pour travailler dans ce noyau urbain.

- Une agglomération de recensement (AR) est formée d'un noyau urbain de 10000 à 99999 habitants et de toutes les villes et municipalités voisines dont $50 \%$ ou plus de la population active fait la navette pour travailler dans ce noyau urbain.

- Une zone d'influence métropolitaine (ZIM) forte est une région où de $30 \%$ à $50 \%$ de la population active fait la navette pour travailler dans une RMR.

- Une ZIM modérée est une région où au moins 5 \% mais moins de $30 \%$ de la population active fait la navette pour travailler dans une RMR ou une AR.
- Une ZIM faible est une région où moins de $5 \%$ de la population active fait la navette pour travailler dans une RMR ou une AR.

- Une zone sans influence métropolitaine (ou nulle) est une région où la population active est restreinte (moins de 40 personnes) ou bien une région dont aucun résident ne fait la navette pour travailler dans une RMR ou une AR.

\section{Sources des données et} méthodes d'analyse

Nous nous sommes servis dans cette étude des données de l'Enquête sur la santé dans les collectivités canadiennes (ESCC), de l'Enquête sur l'accès aux services de santé (EASS), des demandes de paiement des médecins et de la Base de données sur la morbidité hospitalière (BDMH). Ces sources ont permis d'établir plusieurs indicateurs de l'utilisation des services de santé. Pour chaque indicateur, la catégorie urbaine (RMR-AR) a servi de groupe de référence auquel nous avons comparé les diverses catégories rurales (ZIM).

Enquête sur la santé dans les collectivités canadiennes et Enquête sur l'accès aux services de santé

Les données de l'Enquête sur la santé dans les collectivités canadiennes, 2000-2001 (Cycle 1.1), et de l'Enquête sur l'accès aux services de santé ont été analysées en deux étapes. Nous avons tout d'abord effectué des analyses bidimensionnelles pour examiner les différences entre l'utilisation autodéclarée des services de santé dans les collectivités urbaines et rurales. Les taux pour plusieurs indicateurs ont été calculés en fonction du sexe dans les groupes urbains (RMR-AR) et ruraux (toutes les catégories de ZIM) après normalisation selon l'âge. Dans la présente analyse, cette normalisation s'est faite en fonction de la répartition de la population recensée en 2001. Les données ont été pondérées pour tenir compte du plan de sondage complexe et des non-réponses. La méthode du bootstrap a été employée pour calculer des intervalles de confiance (IC) de $95 \%$.

Nous avons ensuite effectué des analyses de régression logistique multivariées pour établir la relation entre le lieu de résidence et l'absence de médecin de famille autodéclarée ainsi qu'entre le lieu de résidence et l'hospitalisation. Le but de cette analyse était de vérifier, après avoir pris en compte diverses variables déterminantes de la santé, si le lieu de résidence influe de façon indépendante sur certains résultats. Nous avons fondé le choix de ces déterminants de la santé sur le cadre théorique d'Anderson ${ }^{2}$, mais avons été freinés par le peu d'information sur ces facteurs contenu dans les bases de données consultées. Comme dans le cas de l'analyse bidimensionnelle, les données ont été pondérées pour tenir compte du plan de sondage complexe et des non-réponses. Nous avons employé la méthode du bootstrap pour calculer des intervalles de confiance de $95 \%$.

Les données de l'ESCC et de l'EASS proviennent d'un échantillon de toutes les subdivisions de recensement (SDR) au Canada. Les deux enquêtes ont été effectuées auprès de personnes de 12 ans et plus (ESCC) ou de 15 ans et plus (EASS), à l'exclusion des personnes vivant dans les réserves indiennes et sur les terres publiques, des résidents d'établissements (p. ex. prisons), des membres à plein temps des Forces canadiennes et des résidents de certaines régions éloignées. Par conséquent, puisqu’à ces régions rurales ou éloignées correspond dans ces enquêtes un nombre inférieur à la réalité de membres de ces sous-populations 
(particulièrement dans le cas de certaines zones sans influence métropolitaine où se trouvent des réserves indiennes très faiblement peuplées), les résultats pourraient ne pas être représentatifs : il faut éviter de tirer des conclusions sur ces sous-populations à partir des résultats généraux de l'ESCC et de l'EASS.

\section{Demandes de paiement des médecins}

L'analyse à l'échelle nationale s'est accompagnée d'une analyse au niveau provincial sur les tendances de l'utilisation des services de santé dans les régions rurales. Nous avons utilisé les données sur la facturation des médecins de Nouvelle-Écosse, d'Ontario et de Colombie-Britannique pour examiner le recours à leurs services au cours des trois périodes de deux ans suivantes : 1997-1998, 1999-2000 et 2001-2002. Dans chacune des trois provinces, les personnes ont été classées selon la méthode des ZIM dans une des cinq catégories géographiques (découpage selon le recensement de 1996 ou celui de 2001) et le lieu de résidence a été défini comme la SDR dans laquelle les personnes vivaient au début de chaque période de deux ans examinée. Ainsi, si un résident d'une zone sans influence métropolitaine a consulté un médecin dans une ZIM faible, nous le considérons dans nos analyses comme faisant partie de la catégorie " ZIM nulle».

Nous avons calculé le taux de consultation médicale selon le lieu de consultation (c'est-à-dire au cabinet, à la clinique externe ou au service d'urgence) ainsi le taux de consultation des médecins de famille (MF), des médecins spécialistes et des chirurgiens (tableau 2). La catégorie des " médecins de famille " comprend les omnipraticiens et les médecins de famille affectés aux services d'urgence. Les services aux hospitalisés, les soins à domicile, les maisons de soins infirmiers et les services de laboratoire - ainsi que les services associés aux soins hospitaliers - ne font pas partie des calculs.

Une consultation médicale a été définie comme une rencontre entre un médecin et un patient par jour. Une seule consultation serait attribuée à une personne pour laquelle un même médecin ferait de multiples demandes de paiement le même jour et pour le même code de diagnostic de la CIM-9*. Plusieurs consultations seraient attribuées à une personne pour laquelle plusieurs médecins feraient des demandes de paiement le même jour. Les factures pro forma ont également été incluses dans l'analyse pour prendre en compte certains médecins, surtout dans les régions nordiques ou éloignées, qui n'ont pas été rémunérés à l'acte, mais selon d'autres modes de paiement. Nous avons exclu les résidents d'une province qui consultent dans une autre province et les services offerts aux patients de l'extérieur de la province.

TABLEAU 2

Groupes de spécialités médicales utilisés dans l'analyse des demandes de paiement des médecins

\begin{tabular}{|c|c|c|}
\hline Médecins de famille & Médecins spécialistes & Chirurgiens \\
\hline \multirow{19}{*}{$\begin{array}{l}\text { Médecine générale, } \\
\text { médecine familiale }\end{array}$} & Rhumatologie & Chirurgie orthopédique \\
\hline & Médecine interne & Chirurgie cardiovasculaire \\
\hline & Cardiologie & Chirurgie générale \\
\hline & Immunologie clinique & Chirurgie thoracique générale \\
\hline & Dermatologie & Neurochirurgie \\
\hline & Gastroentérologie & Obstétrique \\
\hline & Génétique & Ophtalmologie \\
\hline & Gériatrie & Oto-rhino-laryngologie \\
\hline & Hématologie & Chirurgie plastique \\
\hline & Pathologie & Urologie \\
\hline & Neurologie & \\
\hline & Médecine nucléaire & \\
\hline & Pédiatrie & \\
\hline & Physiatrie & \\
\hline & Psychiatrie & \\
\hline & Radiodiagnostic & \\
\hline & Radiothérapie & \\
\hline & Maladies respiratoires & \\
\hline & Anesthésie & \\
\hline
\end{tabular}

Nota : Les chercheurs en médecine et les spécialistes de laboratoire ont été exclus des analyses.

Dans le cas des analyses portant sur la Colombie-Britannique, les consultations auprès des pathologistes et des médecins microbiologistes ont été exclues.
Les données sur les demandes de paiement des médecins sont d'ordre administratif et se limitent donc aux services payés à l'acte ou facturés pro forma. Puisque certains services du médecin ne sont pas détaillés, comme dans le cas des médecins rémunérés selon d'autres modes de paiement, et que les codes de gestion ne permettent pas toujours de distinguer les divers types de services offerts (comme les services de santé mentale), les résultats peuvent ne pas être représentatifs de la réalité.

Nous avons calculé, pour les trois périodes de deux ans, et pour chaque sexe, les indi-

- le taux de consultation auprès des médecins, soit le nombre moyen de consultations auprès de l'ensemble des médecins pour 1000 résidents de la région; cateurs suivants : 
- le taux de consultation auprès des MF, soit le nombre moyen de consultations auprès des MF pour 1000 résidents de la région;

- le taux de consultation auprès des spécialistes, soit le nombre moyen de consultations auprès des spécialistes et des chirurgiens pour 1000 résidents de la région;

- le taux de consultation auprès des médecins selon le type de maladie, soit le nombre moyen de consultations auprès des médecins selon le code de diagnostic de la CIM-9 pour 1000 résidents de la région.

Tous les taux ont été normalisés selon l'âge en prenant pour référence la structure par âge de la population qui prévalait lors du recensement de 1991. Les ratios des taux ont été calculés à l'aide du taux normalisé selon l'âge de la population dans la RMR-AR. Enfin, nous avons aussi examiné des diagnostics particuliers en fonction de leur pertinence à l'égard des populations rurales et de leur incidence sur la santé de cette population ainsi que selon la disponibilité des données (tableau 3).

\section{Base de données sur la morbidité hospitalière}

La Base de données sur la morbidité hospitalière (BDMH), gérée par l'ICIS, contient des données nationales sur les soins de courte durée selon le diagnostic et l'acte médical. Les données sont présentées selon la région où réside le patient et non selon celle où l'hôpital est situé. Elles révèlent ainsi la fréquence à laquelle les résidents d'une région donnée reçoivent des soins médicaux plutôt que le volume des services fournis par chaque hôpital. Les données de la BDMH sont fondées sur les congés accordés par l'hôpital (plutôt que sur les admissions). Seules les personnes vivantes au moment de leur congé sont incluses dans l'analyse : les " congés » des morts-nés et des donneurs décédés sont exclus de la base de données. En sont exclues aussi les interventions d'un jour (telles que les chirurgies d'un jour) et les consultations au service d'urgence. Pour la présente analyse, les données sur les congés des nouveaux-nés ont aussi été exclues.

La BSMH contient les données des exercices de 1994-1995 à 2000-2001 classées selon la CIM-9 et la CCA*,†. En 2000-2001, les établissements du Yukon, de la Nouvelle-Écosse, de l'Île-du-Prince-Édouard

TABLEAU 3

Diagnostics utilisés dans la comparaison des risques relatifs des visites chez le médecin et des hospitalisations, pour populations urbaines et rurales

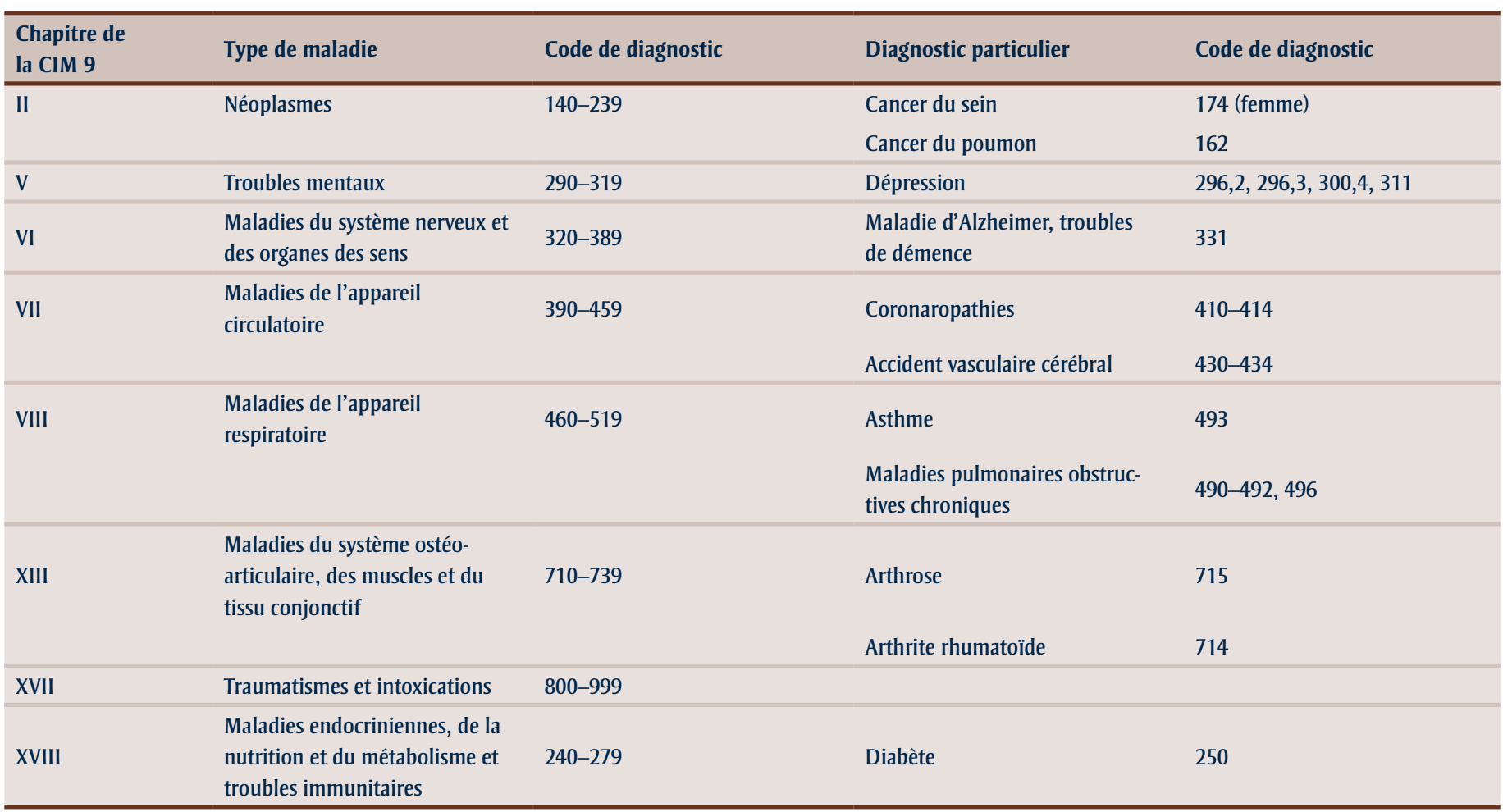

Source : Organisation mondiale de la santé.

CIM-9 : Classification statistique internationale des maladies, traumatismes et causes de décès, $9^{\mathrm{e}}$ révision. 
et de Terre-Neuve-et-Labrador, et certains établissements de la Saskatchewan, sont passés à la $\mathrm{CIM}-10-\mathrm{CA}^{\ddagger}$ et à la $\mathrm{CCI}^{\S}$. Par conséquent, à partir de 2001-2002, la base de données contient des données classées selon les deux systèmes.

Nous avons extrait de la BDMH les données sur les congés des établissements de la Nouvelle-Écosse, de l'Ontario et de la Colombie-Britannique ainsi que de l'ensemble du Canada, à l'exception du Québec ${ }^{\prime}$. Les données ont fait l'objet d'une analyse de cohorte et ont été extraites suivant le code de diagnostic selon lequel elles ont été communiquées (CIM-9) ou CIM-10-CA). L'incidence de l'utilisation de systèmes de classification différents sur les tendances rurales et urbaines nationales demeure inconnue pour l'instant. La CIM-10-CA se distingue de la CIM-9 à plusieurs égards, le premier système étant plus détaillé que le second. Nous avons appliqué le découpage géographique correspondant au recensement de 1996 aux données de 2001-2002 (du 1 ${ }^{\text {er }}$ avril 2001 au 31 mars 2002) que nous avons extraites. Le lieu de résidence en milieu rural a été établi selon les ZIM définies plus haut.

Les taux de congé et les données sur la durée de l'hospitalisation sont fondés sur le nombre de congés correspondant à des soins de courte durée au Canada en 2001-2002. Si une personne a été admise plus d'une fois dans un établissement de soins de courte durée et a obtenu son congé, elle sera comptée plus d'une fois. En outre, nous avons calculé les taux de congé de divers types de maladies suivant les codes de diagnostic de la CIM-9. Les sections où ces codes sont utilisés ont été produites en fonction de critères d'extraction de données portant sur le " diagnostic principal ». Pour qu'un diagnostic particulier réponde à ces critères, il doit être consigné dans la base de données sur les congés des patients comme étant la raison principale pour laquelle le patient a été hospitalisé. Tous les indicateurs ont été normalisés selon le sexe et selon l'âge en prenant pour référence la population au moment du recensement de 1991. La signification statistique des taux de congé des établissements de soins de courte durée a été vérifiée au moyen de la méthode de Byar et est fondée sur l'hypothèse d'une distribution de Poisson ${ }^{23}$.

\section{Notes statistiques générales}

Tout au long du présent rapport, les estimations sont fournies avec des intervalles de confiance (IC) à $95 \%$. Les statistiques doivent être considérées comme significatives si les intervalles de confiance à $95 \%$ ne se chevauchent pas. Les taux décrits comme étant "significativement différents » sont différents au sens statistique avec un intervalle de confiance à $95 \%$. Comme la faible population de certaines ZIM faibles et nulles limite parfois la quantité de données pour calculer les taux, le degré d'incertitude lié à ces taux est plus élevé dans ces zones que dans les régions plus peuplées (c'est-à-dire les RMR et les AR). Par conséquent, les intervalles de confiance ont été calculés et les taux présentés de façon à ce que le degré d'incertitude soit clairement énoncé. Ces intervalles de confiance ne décrivent pas l'incertitude associée au biais potentiel, par exemple l'incertitude dans l'identification exacte des SDR.

Le premier découpage géographique retenu dans l'analyse est celui du recensement de 1996, car c'est celui dont nous disposions au début de la recherche. Les différences de découpage entre les recensements présentes dans les diverses analyses du rapport résultent du décalage entre ces analyses. Par exemple, les demandes d'accès aux données sur la facturation des médecins de Colombie-Britannique et à celles de la BDMH ont été effectuées au début du projet. Par ailleurs, une comparaison a permis d'établir qu'il était beaucoup plus judicieux de cerner les lieux géographiques de la Nouvelle-Écosse grâce aux frontières du recensement de 2001 (2 \% des SDR n'avaient pas de code) plutôt qu'à celles du recensement de 1996 (environ $18 \%$ des SDR n'avaient pas de code). Nous avons mis cela sur le compte de la création de nouveaux codes postaux au cours de la période suivant la définition des limites géographiques du recensement de 1996. Dans la mesure du possible, les données extraites ultérieurement et les analyses dont elles ont fait l'objet ont pris en compte les frontières du recensement de 2001 .

Enfin, comme la présente publication nomme diverses sources, il faut faire preuve de circonspection dans la comparaison des données de ces sources, car elles pourraient porter sur des concepts différents même si elles relèvent d'un même ensemble.

\section{Résultats}

\section{Services de soins de santé - accès et utilisation à l'échelle du Canada}

\section{Accès}

Selon l'EASS 2000-2001, c'est dans des proportions similaires que les répondants des régions urbaines et rurales (sauf des ZIM nulles) ont dit avoir un médecin de famille (RMR-AR : 87,3 \%; ZIM fortes, modérées, faibles : de 88,3\% à 90,6\%; ZIM nulles : 76,1 \%). Parmi ceux qui n’ont pas de médecin de famille, de $35 \%$ à $61 \%$ des résidents des régions rurales, contre $13,2 \%$ de ceux des régions urbaines, ont dit ne pas avoir de médecin de famille parce qu'il n’y en avait aucun de disponible. À l'exception

\footnotetext{
* Classification canadienne des actes diagnostiques, thérapeutiques et chirurgicaux.

† On peut obtenir les données antérieures à l'exercice 1994-1995 auprès de la Division des statistiques sur la santé, Statistique Canada.

₹ International Classification of Diseases, 10th revision - Canadian Enhancement (améliorations canadiennes à la CIM-10).

$\S$ Classification canadienne des interventions en santé.

| Au moment de l'analyse, les données sur les congés communiquées par le Québec n'incluaient que la région de tri d'acheminement (trois premiers caractères) du code postal. Par conséquent, les correspondances avec les diverses catégories de ZIM n'ont pu être déterminées, car les six caractères du code postal sont nécessaires pour le classement.
} 
FIGURE 2

Proportion (normalisée selon l'âge) de Canadiens de $\mathbf{1 5}$ ans et plus qui ont eu besoin de soins de routine ou de suivi, selon le lieu de résidence, 2000-2001

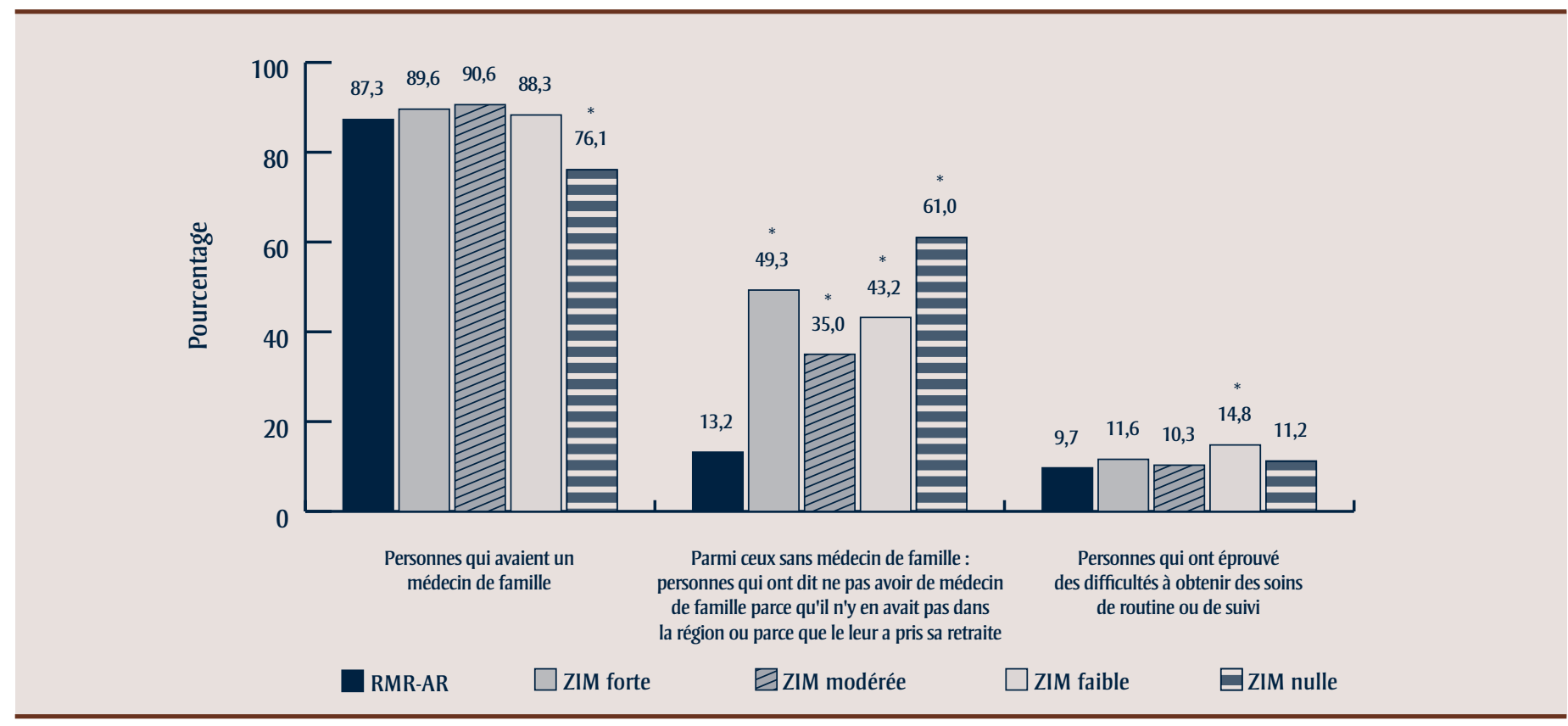

Source : Enquête sur l'accès aux services de santé 2000-2001, Statistique Canada.

Abréviations : AR, agglomération de recensement; RMR, région métropolitaine de recensement; ZIM, zone d'influence métropolitaine.

* Statistiquement significatif à $p<0,05$; le groupe de référence est la RMR-AR.

des ZIM nulles, où un nombre significativement plus élevé de résidents ont dit avoir des difficultés à obtenir des soins de routine $(14,8 \%)$, les régions urbaines et rurales comptent des proportions similaires de répondants disant éprouver ces difficultés (de 9,7\% à 11,6\%) (figure 2). Une fois le sexe, l'âge, les maladies chroniques, les comportements liés à la santé et les facteurs sociodémographiques et économiques pris en compte, l'analyse multivariée révèle que le lieu de résidence a un effet indépendant (rapport de cotes $[\mathrm{RC}]=2,65$, IC à $95 \%=1,51$ à 4,64 ) sur le fait de ne pas avoir de médecin de famille seulement dans les ZIM nulles.

La proportion des répondants à l'EASS qui ont dit avoir dû recourir à des soins immédiats pour un problème de santé mineur au cours de l'année précédant l'enquête était significativement plus élevée dans les ZIM faibles que dans les régions urbaines (RMR-AR : 32,9 \%; ZIM faibles : 39,6 \%). De plus, la proportion des répondants qui ont déclaré avoir éprouvé des difficultés à obtenir des soins immédiats parce qu'ils n'étaient pas offerts dans la région, qu'ils n'étaient pas offerts au moment où ils étaient nécessaires ou à cause de problèmes de transport était significativement plus élevée dans les ZIM nulles (RMR-AR : 8,8 \%; ZIM nulles : 61,4\%) (figure 3).

\section{Temps d'attente avant de consulter un médecin spécialiste ou de subir une chirurgie non urgente}

En ce qui a trait au temps d'attente concernant les soins spécialisés, de 57,0 \% à $77,7 \%$ des répondants à l'EASS ont dit avoir attendu plusieurs mois avant d'obtenir ce type de soins en 2000-2001, et les proportions ne varient pas significativement selon le lieu de résidence (figure 4). La proportion de Canadiens qui ont trouvé que les temps d'attente étaient inacceptables était significativement plus élevée dans les ZIM fortes que dans les régions urbaines (RMR-AR : 31,3\%; ZIM fortes : $47,5 \%)$. La proportion des résidents des ZIM nulles qui ont trouvé les temps d'attente inacceptables était aussi élevée que celle des résidents des ZIM fortes, mais elle n'était pas significative sur le plan statistique.

\section{Utilisation des ressources et des services de santé}

Dans l'ensemble, le ratio de médecins de famille (MF) pour 10000 habitants était plus élevé dans les régions urbaines que dans les régions rurales $(10,1$ contre 6,9$)$, quoique dans les ZIM faibles et les RRPV des Territoires (les régions du Yukon, des Territoires-du-Nord-Ouest et du Nunavut autres que les RMR-AR, c'est-à-dire toutes les régions des Territoires sauf Yellowknife et Whitehorse), ce ratio ait été comparable voire supérieur à celui des régions urbaines (respectivement 10,2 et 14,7 pour 10000 habitants). L'écart entre les ratios de spécialistes était beaucoup plus important : 11,0 pour 10000 habitants dans les régions urbaines contre 1,0 pour 10000 habitants dans l'ensemble des régions rurales (tableau 4). 
FIGURE 3

Proportion (normalisée selon l'âge) de Canadiens de 15 ans et plus ayant eu besoin de soins immédiats pour un problème de santé mineur au cours de l'année précédente, selon le lieu de résidence, 2000-2001

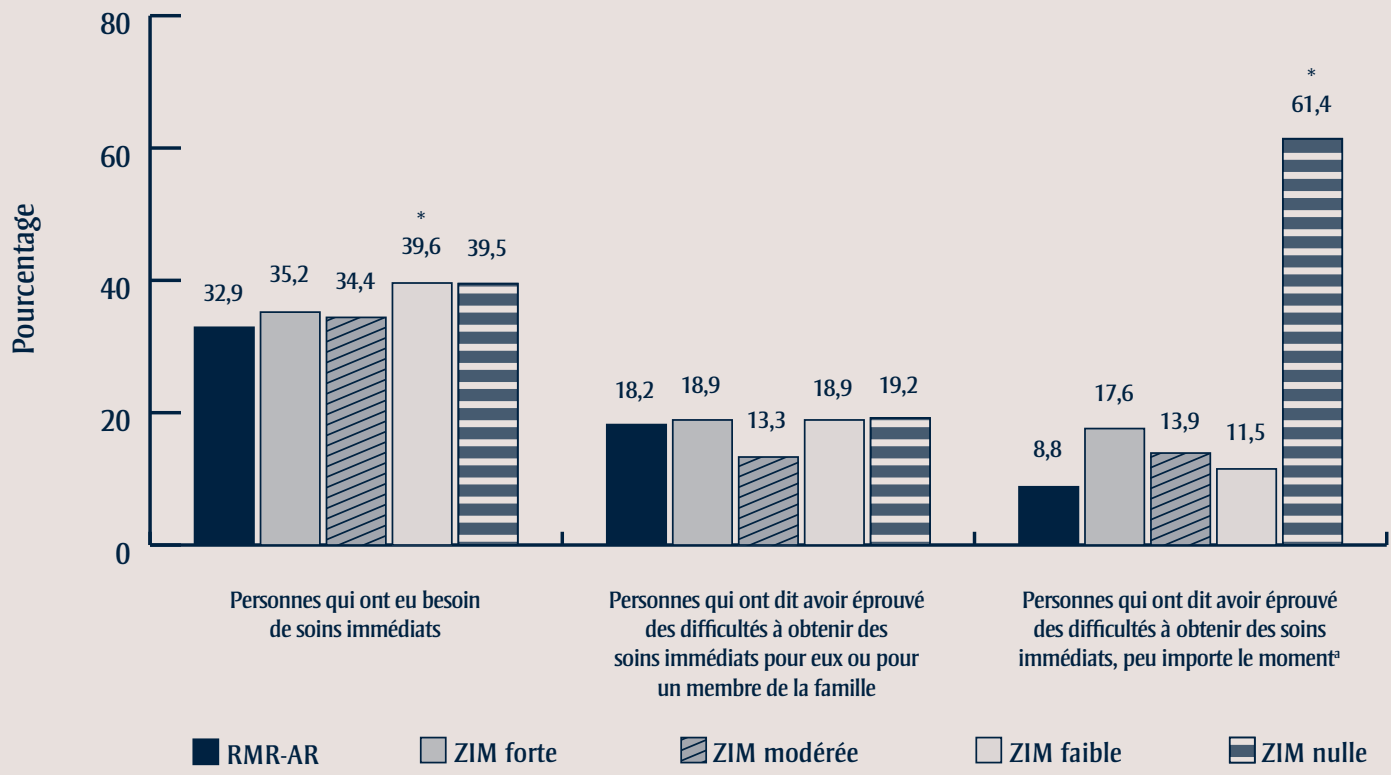

Source : Enquête sur l'accès aux services de santé 2000-2001, Statistique Canada.

Abréviations : AR, agglomération de recensement; RMR, région métropolitaine de recensement; ZIM, zone d'influence métropolitaine.

* Statistiquement significatif à $p<0,05$; le groupe de référence est la RMR-AR.

a Raisons pour lesquelles les soins de santé n'ont pas été obtenus : soins non offerts dans la région; soins non offerts au moment ils étaient nécessaires; problèmes de transport.

FIGURE 4

Proportion (normalisée selon l'âge) de Canadiens de 15 ans et plus ayant déclaré avoir attendu plusieurs mois avant d'obtenir des soins spécialisés ou de subir une chirurgie non urgente, selon le lieu de résidence, 2000-2001

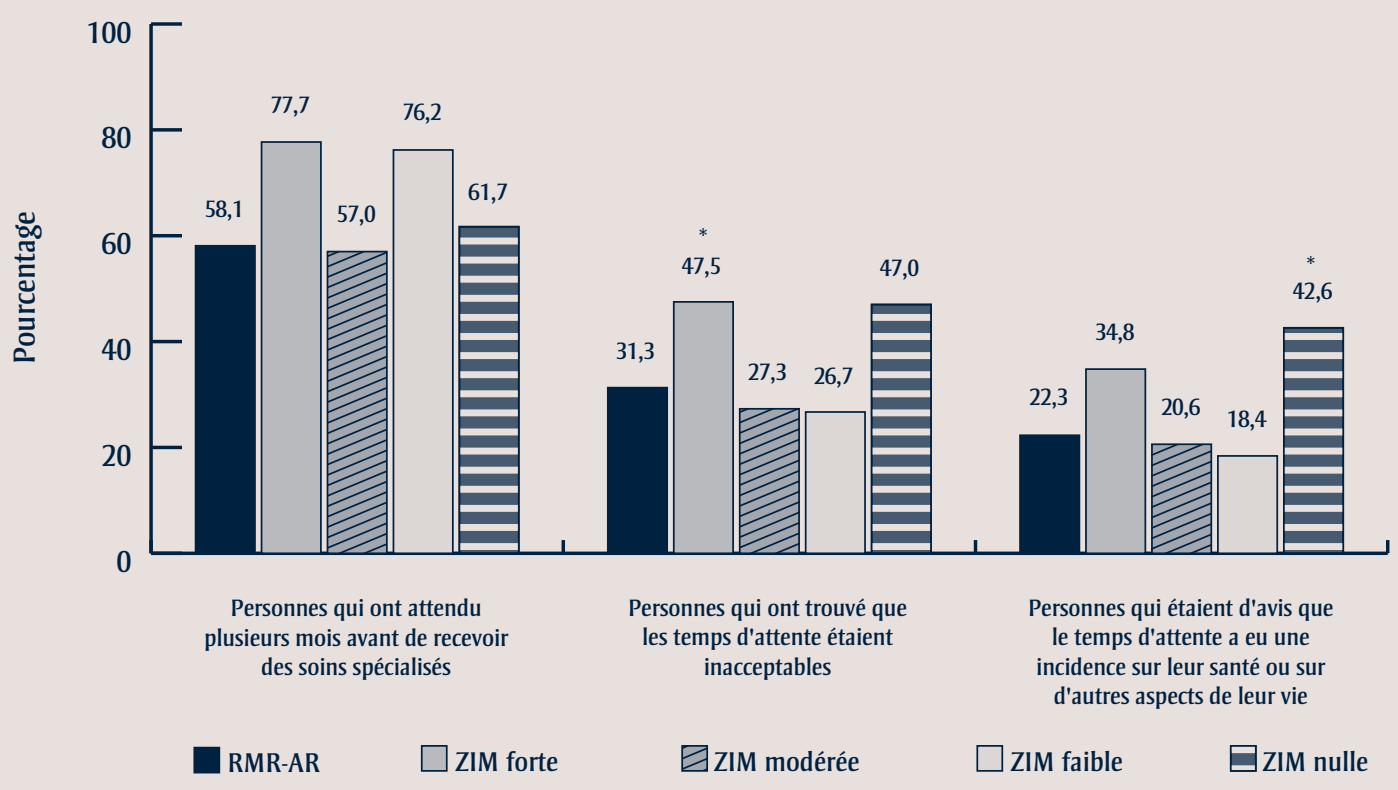

Source : Enquête sur l'accès aux services de santé 2000-2001, Statistique Canada.

Abréviations : AR, agglomération de recensement; RMR, région métropolitaine de recensement; ZIM, zone d'influence métropolitaine.

* Statistiquement significatif à $p<0,05$; le groupe de référence est la RMR-AR. 
TABLEAU 4

Nombre de médecins ${ }^{\mathrm{a}}$ et ratios de médecins pour 10000 habitants, selon le type de médecin et le lieu de résidence, 2001

\begin{tabular}{lccccc}
\hline & Médecins de famille & $\begin{array}{c}\text { Médecins de famille } \\
\text { pour } \mathbf{1 0} \mathbf{0 0 0} \text { habitants }\end{array}$ & $\begin{array}{c}\text { Médecins spécialistes } \\
\text { et chirurgiens }\end{array}$ & $\begin{array}{c}\text { Spécialistes pour } \\
\mathbf{1 0} \mathbf{0 0 0} \text { habitants }\end{array}$ & $\begin{array}{c}\text { Estimation de } \\
\text { l'effectif de la population }\end{array}$ \\
\hline $\begin{array}{l}\text { Région urbaine (RMR-AR) } \\
\text { Ensemble des ZIM } \\
\text { et des RRPV }\end{array}$ & 24133 & 10,1 & 26276 & 11,0 & 23929310 \\
ZIM forte & 4244 & 6,9 & 626 & 1,0 & 6447995 \\
ZIM modérée & 533 & 3,5 & 70 & 0,5 & 1524335 \\
ZIM faible & 1466 & 6,4 & 172 & 0,8 & 2283225 \\
ZIM nulle & 2008 & 10,2 & 337 & 31,7 & 1961235 \\
RRPV des territoires & 156 & 4,6 & 16 & 0,9 & 2,9 \\
\hline Total & 81 & 14,7 & 26902 & 8,9 & 100110 \\
\hline
\end{tabular}

Source : Base de données médicales Southam, Institut canadien d'information la santé, 2001.

Abréviations : AR, agglomération de recensement; RMR, région métropolitaine de recensement; ZIM, zone d'influence métropolitaine; RRPV, région rurale et petite ville.

a Exclut les résidents et les médecins selon la méthodologie de non-publication (consulter les notes méthodologiques du rapport de 2003 de l’ICIS intitulé Nombre, répartition et migration des médecins canadiens); comprend les médecins qui fournissent des services cliniques et non cliniques; comprend les omnipraticiens, accrédités ou non par le CMFC, les spécialistes accrédités à l'étranger et les autres spécialistes non accrédités.

b Régions du Yukon, des Territoires-du-Nord-Ouest et du Nunavut autres que les RMR-AR, c'est-à-dire toutes les régions des Territoires, sauf Yellowknife et Whitehorse.

FIGURE 5

Proportion (normalisée selon l'âge) des hommes de 12 ans et plus ayant déclaré avoir consulté un médecin au cours des 12 derniers mois, selon le type de médecin et le lieu de résidence, Canada, 2000-2001

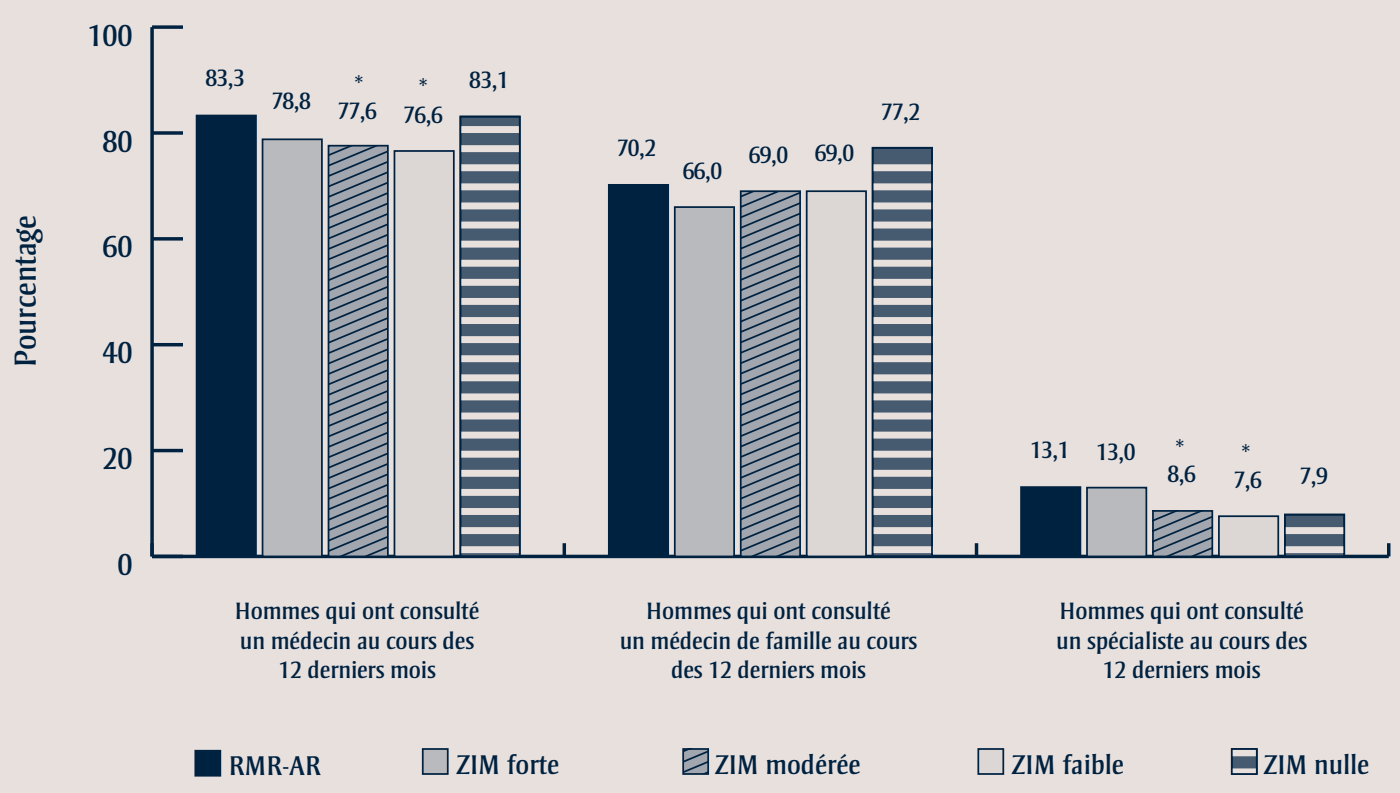

Source : Enquête sur la santé dans les collectivités canadiennes, 2000-2001, Statistique Canada.

Abréviations : AR, agglomération de recensement; RMR, région métropolitaine de recensement; ZIM, zone d’influence métropolitaine.

* Statistiquement significatif à $p<0,05$; le groupe de référence est la RMR-AR. 
Les données de l'EASS 2000-2001 révèlent que plus de $75 \%$ des répondants de sexe masculin ont déclaré avoir consulté un médecin au cours des 12 mois précédant l'enquête. Il n'y avait pas de différence significative sur le plan statistique en fonction du lieu de résidence des hommes en ce qui concerne la consultation d'un médecin de famille (de 66,0 \% à 77,2\%), en revanche les hommes des ZIM modérées et faibles ont été moins nombreux (respectivement $8,6 \%$ et $7,6 \%$ ) que ceux des régions urbaines $(13,1 \%)$ à consulter un spécialiste (figure 5).

Il n’y avait pas de différence statistiquement significative liée au lieu de résidence en ce qui concerne la proportion de femmes ayant déclaré avoir consulté un médecin de famille, à l'exception des ZIM faibles, où cette proportion a été plus élevée (RMR-AR : 74,6 \%; ZIM faible : 78,5 \%). La proportion de femmes ayant déclaré avoir consulté un spécialiste a été significativement plus faible dans les ZIM faibles que dans les régions urbaines (RMR-AR : 15,1 \%; ZIM faible : 8,4 \%) (figure 6).

Parmi les répondants de l’ESCC 2000-2001 ayant déclaré avoir consulté un médecin au cours des 12 mois précédant l'enquête, la proportion d'hommes n'ayant pas consulté de médecin de famille était significativement plus élevée dans les régions rurales. Les femmes affichent une tendance similaire, sauf qu'il n'y avait pas de différence significative sur le plan statistique entre celles des ZIM fortes et celles

FIGURE 6

Proportion (normalisée selon l'âge) des femmes de 12 ans et plus ayant déclaré avoir consulté un médecin au cours des 12 derniers mois, selon le type de médecin et le lieu de résidence, Canada, 2000-2001

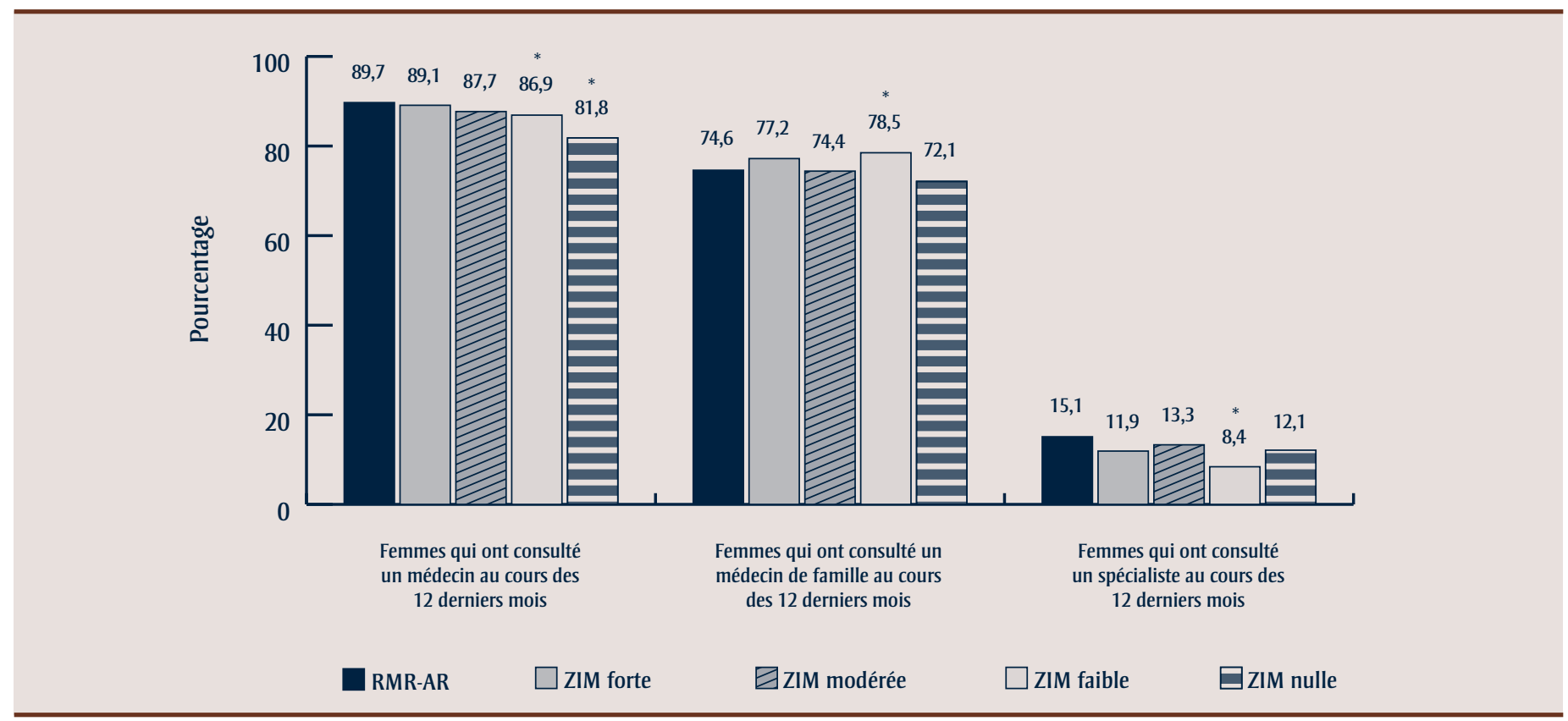

Source : Enquête sur la santé dans les collectivités canadiennes, 2000-2001, Statistique Canada.

Abréviations : AR, agglomération de recensement; RMR, région métropolitaine de recensement; ZIM, zone d'influence métropolitaine.

* Statistiquement significatif à $p<0,05$; le groupe de référence est la RMR-AR. 
des régions urbaines. Des proportions similaires d'hommes des régions rurales et urbaines ont dit avoir consulté un médecin de famille quatre fois ou plus. Toutes catégories géographiques confondues, la proportion de femmes ayant consulté un médecin de famille quatre fois ou plus a été supérieure à celle des hommes. Les hommes des régions rurales ont été moins nombreux que ceux des régions urbaines à déclarer avoir consulté au moins une fois un autre type de médecin, sauf dans le cas des ZIM fortes. Les femmes de toutes les régions rurales ont été moins nombreuses à mentionner avoir consulté un autre type de médecin (tableau 5).

Selon les résultats de l'ESCC 2000-2001, les résidents des ZIM modérées, faibles et nulles étaient significativement plus nombreux que ceux des régions urbaines à déclarer avoir consulté une infirmière (tableau 6). La proportion des répondants ayant consulté un dentiste ou reçu des soins de santé communautaire (c'est-à-dire des services fournis en dehors de l'hôpital ou du cabinet du médecin) dans les 12 mois précédant l'enquête était beaucoup plus faible dans les régions rurales que dans les régions urbaines, sauf en ce qui concerne

\section{TABLEAU 5}

Proportion (normalisée selon l’âge) de Canadiens de 12 ans et plus qui ont déclaré avoir consulté un médecin au cours des 12 derniers mois, selon le lieu de résidence, 2000-2001

\begin{tabular}{|c|c|c|c|c|c|c|}
\hline Consultations d'un médecin & & RMR-AR & ZIM forte & ZIM modérée & ZIM faible & ZIM nulle \\
\hline \multirow{2}{*}{$\begin{array}{l}\text { Personnes qui ont déclaré n'avoir } \\
\text { consulté aucun médecin de famille }\end{array}$} & Hommes & $26,5(25,9-27,2)$ & $31,6(29,7-33,4)^{*}$ & $29,4(27,9-30,8)^{*}$ & $29,2(28,0-30,5)^{*}$ & $33,6(29,8-37,5)^{*}$ \\
\hline & Femmes & $15,3(14,8-15,8)$ & $16,5(15,0-17,9)$ & $17,4(16,2-18,6)^{*}$ & $17,3(16,3-18,3)^{*}$ & $20,3(17,2-23,4)^{*}$ \\
\hline $\begin{array}{l}\text { Personnes qui ont déclaré avoir consulté } \\
\text { un médecin de famille } 4 \text { fois ou plus }\end{array}$ & Femmes & $34,4(33,8-35,4)$ & $33,2(31,4-35,0)$ & $34,9(33,5-36,2)$ & $37,2(36,0-38,4)^{*}$ & $38,3(34,9-41,6)$ \\
\hline $\begin{array}{l}\text { Personnes qui ont déclaré avoir consulté un } \\
\text { autre type de médecin au moins une fois }\end{array}$ & Hommes & $24,0(23,4-24,6)$ & $23,5(21,8-25,1)$ & $20,2(18,9-21,4)^{*}$ & $19,0(17,9-20,0)^{*}$ & $16,1(13,4-18,9)^{*}$ \\
\hline
\end{tabular}

Source : Enquête sur la santé dans les collectivités canadiennes, 2000-2001, Statistique Canada.

Abréviations : AR, agglomération de recensement; RMR, région métropolitaine de recensement; ZIM, zone d’influence métropolitaine.

* Statistiquement significatif à $p<0,05$; le groupe de référence est la RMR-AR.

\section{TABLEAU 6}

Proportion (normalisée selon l'âge) des personnes de 15 ans et plus qui ont déclaré avoir consulté un dentiste, une infirmière ou d'autres fournisseurs de soins de santé, selon le lieu de résidence et le sexe, Canada, 2000-2001

\begin{tabular}{|c|c|c|c|c|c|c|}
\hline \multicolumn{2}{|c|}{ Consultations d'un fournisseur de soins de santé } & RMR-AR & ZIM forte & ZIM modérée & ZIM faible & ZIM nulle \\
\hline \multirow{2}{*}{$\begin{array}{l}\text { Personnes qui ont dit avoir consulté } \\
\text { une infirmière au moins une fois }\end{array}$} & Hommes & $7,8(7,4-8,1)$ & $7,7(6,7-8,8)$ & $9,1(8,2-10,0)^{*}$ & $10,4(9,5-11,3)^{*}$ & $10,5(8,4-12,6)^{*}$ \\
\hline & Femmes & $11,6(11,2-12,0)$ & $12,5(11,3-13,8)$ & $13,1(12,2-14,1)^{*}$ & $14,1(13,3-15,0)^{*}$ & $20,3(17,3-23,4)^{*}$ \\
\hline \multirow{2}{*}{$\begin{array}{l}\text { Personnes qui ont dit avoir consulté } \\
\text { un dentiste au moins une fois }\end{array}$} & Hommes & $59,8(59,1-60,6)$ & $54,1(25,2-56,1)^{*}$ & $46,6(45,1-48,2)^{*}$ & $48,6(47,2-49,9)^{*}$ & $44,1(40,3-47,8)^{*}$ \\
\hline & Femmes & $64,2(63,6-64,8)$ & $58,5(56,7-60,3)^{*}$ & $53,6(52,2-55,1)^{*}$ & $53,5(52,3-54,7)^{*}$ & $45,4(41,7-49,1)^{*}$ \\
\hline \multirow{2}{*}{$\begin{array}{l}\text { Personnes qui ont reçu des soins } \\
\text { de santé communautaire au cours } \\
\text { des } 12 \text { derniers mois }\end{array}$} & Hommes & $15,4(14,2-16,7)$ & $9,5(7,1-12,0)^{*}$ & $11,1(8,6-13,6)^{*}$ & $10,1(8,1-12,0)^{*}$ & $10,7(5,0-16,4)$ \\
\hline & Femmes & $19,0(17,9-20,1)$ & $14,2(11,6-16,7)^{*}$ & $12,5(10,5-14,5)^{*}$ & $13,6(11,7-15,4)^{*}$ & $15,6(9,7-21,5)$ \\
\hline
\end{tabular}

Source : Enquête sur la santé dans les collectivités canadiennes, 2000-2001, Statistique Canada.

Abréviations : AR, agglomération de recensement; RMR, région métropolitaine de recensement; ZIM, zone d'influence métropolitaine.

* Statistiquement significatif à $p<0,05$; le groupe de référence est la RMR-AR.

TABLEAU 7

Taux de congé d'hôpital (normalisés selon l'âge) pour 1000 habitantsa , selon le lieu de résidence et le sexe, 2001-2002

\begin{tabular}{|c|c|c|c|c|c|}
\hline Sexe & RMR-AR & ZIM forte & ZIM modérée & ZIM faible & ZIM nulle \\
\hline Hommes & $67,8(67,7-68,0)$ & $72,0(71,4-72,7)^{*}$ & $83,8(83,2-84,4)^{*}$ & $107,2(106,5-107,8)^{*}$ & $116,6(115,0-118,3)^{*}$ \\
\hline Femmes & $88,2(88,0-88,4)$ & $98,6(97,7-99,4)^{*}$ & $111,1(110,3-111,8)^{*}$ & $140,0(139,2-140,7)^{*}$ & $161,3(159,2-163,4)^{*}$ \\
\hline
\end{tabular}

Source : Base de données sur la morbidité hospitalière, 2001-2002, Institut canadien d'information sur la santé.

Abréviations : AR, agglomération de recensement; RMR, région métropolitaine de recensement; ZIM, zone d'influence métropolitaine.

a Les totaux pour l'ensemble du Canada excluent le Québec faute de codes géographiques suffisants dans les données de base.

* Statistiquement significatif à $p<0,05$; le groupe de référence est la RMR-AR. 
les soins de santé communautaire dans les ZIM nulles (tableau 6).

En ce qui a trait aux soins hospitaliers, les données de la BDMH pour 2001-2002 révèlent des taux d'hospitalisation plus élevés chez les résidents de toutes les régions rurales que chez les résidents des régions urbaines, et les différences entre les régions urbaines et l'ensemble des régions rurales étaient statistiquement toutes significatives (tableau 7).

Les résultats de l'ESCC 2000-2001 concordent avec ces constatations : une proportion significativement plus élevée de résidents des régions rurales ont dit avoir reçu des soins hospitaliers dans les 12 mois précédant l'enquête. En général, la proportion des personnes hospitalisées différait peu d'une région à l'autre, mais un nombre significativement plus élevé d'habitants des régions rurales ont reçu des soins en clinique externe dans les ZIM fortes (femmes), les ZIM modérées (hommes), les ZIM faibles (hommes et femmes) et les ZIM nulles (femmes).

La durée de l'hospitalisation augmente avec l'âge, mais elle diminue de façon inversement proportionnelle au degré de ruralité dans tous les groupes d'âge, sans égard au sexe. Chez les hommes, les variations dans les durées d'hospitalisation sont en lien avec l'âge : alors que la durée moyenne de l'hospitalisation des hommes de 20 à 44 ans était similaire dans les régions urbaines et rurales, les hommes de 45 à 64 ans des ZIM faibles et nulles étaient hospitalisés significativement moins longtemps que ceux du même groupe d'âge des régions urbaines. De même, la durée moyenne de l'hospitalisation des hommes de 65 à 74 ans des régions rurales était significativement plus courte que celle des hommes du même groupe d'âge des régions urbaines. La durée moyenne de l'hospitalisation des hommes de 74 ans ou plus était elle aussi plus courte chez les résidents des ZIM que chez les hommes du même groupe d'âge des régions urbaines (tableau 8).

La durée moyenne de l'hospitalisation des femmes des régions rurales était plus courte que celle des femmes des régions urbaines, et ce sont les femmes des ZIM nulles qui ont dit avoir été hospitalisées le moins longtemps. Une exception : aucune différence n’a été observée entre les

\section{TABLEAU 8}

Durée moyenne d'hospitalisation, en jours, selon le groupe d'âge, le sexe et le lieu de résidence, Canada', 2001-2002

\begin{tabular}{|c|c|c|c|c|c|c|}
\hline Groupe d'âge & Sexe & RMR-AR & ZIM forte & ZIM modérée & ZIM faible & ZIM nulle \\
\hline \multirow{2}{*}{$\begin{array}{l}\text { Tous les } \\
\text { groupes d'âge }\end{array}$} & Hommes & $8,58(7,97-9,18)$ & $7,43(6,91-7,95)^{*}$ & $6,85(6,58-7,19)^{*}$ & $6,65(6,33-6,96)^{*}$ & $6,29(5,97-6,60)^{*}$ \\
\hline & Femmes & $8,28(7,92-8,64)$ & $6,87(6,53-7,21)^{*}$ & $6,92(6,63-7,20)^{*}$ & $6,27(6,04-6,49)^{*}$ & $6,17(5,83-6,50)^{*}$ \\
\hline $20-44$ & Femmes & $5,33(5,07-5,59)$ & $4,58(4,17-4,99)^{*}$ & $4,26(4,04-4,48)^{*}$ & $4,30(3,91-4,69)^{*}$ & $4,01(3,71-4,31)^{*}$ \\
\hline $45-64$ & Hommes & $8,24(7,69-8,79)$ & $7,17(6,08-8,26)$ & $7,21(6,51-7,91)$ & $6,43(6,09-6,76)^{*}$ & $6,15(5,64-6,67)^{*}$ \\
\hline $65-74$ & Femmes & $10,9(10,0-11,8)$ & $8,23(7,59-8,87)^{*}$ & $9,74(8,24-11,2)$ & $8,12(7,29-8,95)^{*}$ & $8,15(7,06-9,24)^{*}$ \\
\hline \multirow{2}{*}{$75+$} & Hommes & $14,5(13,7-15,2)$ & $12,6(11,2-14,1)$ & $10,46(9,85-11,06)^{*}$ & $11,03(10,1-12,0)^{*}$ & $9,93(8,90-11,0)^{*}$ \\
\hline & Femmes & $16,3(14,8-17,8)$ & $12,0(11,0-13,1)^{*}$ & $12,2(11,4-13,0)^{*}$ & $10,9(10,2-11,6)^{*}$ & $10,5(9,27-11,7)^{*}$ \\
\hline
\end{tabular}

Source : Base de données sur la morbidité hospitalière, 2001-2002, Institut canadien d'information sur la santé.

Abréviations : AR, agglomération de recensement; RMR, région métropolitaine de recensement; ZIM, zone d’influence métropolitaine.

a Les totaux pour l'ensemble du Canada excluent le Québec faute de codage géographique utilisable dans les données de base.

* Statistiquement significatif à $p<0,05$; le groupe de référence est la RMR-AR. 
TABLEAU 9

Rapports de cotes corrigés établissant le lien entre le lieu de résidence et l’hospitalisation au cours des 12 derniers mois, $2000-2001$

\begin{tabular}{|c|c|c|c|c|}
\hline & & \multicolumn{3}{|c|}{ Rapport de cotes (intervalles de confiance à $95 \%$ a) } \\
\hline & & Hommes & Femmes & Ensemble \\
\hline \multirow[t]{5}{*}{ Lieu de résidence } & RMR-AR & 1,0 & 1,0 & 1,0 \\
\hline & ZIM forte & $1,06(0,90-1,25)$ & $1,11(0,96-1,28)$ & $1,09(0,98-1,22)$ \\
\hline & ZIM modérée & $1,10(0,96-1,25)$ & $1,10(0,98-1,22)$ & $1,10(1,01-1,20)^{*}$ \\
\hline & ZIM faible & $1,18(1,04-1,33)^{*}$ & $1,16(1,06-1,28)^{*}$ & $1,18(1,09-1,27)^{*}$ \\
\hline & ZIM nulle & $1,35(1,05-1,73)^{*}$ & $1,16(0,93-1,45)$ & $1,27(1,08-1,49)^{*}$ \\
\hline \multirow[t]{2}{*}{ Sexe } & Homme & & & 1,0 \\
\hline & Femme & - & - & $1,46(1,37-1,55)^{*}$ \\
\hline \multirow[t]{2}{*}{ Âge } & $<45$ ans & 1,0 & 1,0 & 1,0 \\
\hline & $\geq 45$ ans & $1,36(1,22-1,52)^{*}$ & $0,56(0,52-0,61)^{*}$ & $0,78(0,73-0,84)^{*}$ \\
\hline \multirow[t]{2}{*}{ Race } & De race blanche & 1,0 & 1,0 & 1,0 \\
\hline & Pas de race blanche & $0,61(0,48-0,77)^{*}$ & $0,76(0,65-0,90)^{*}$ & $0,70(0,61-0,81)^{*}$ \\
\hline \multirow[t]{4}{*}{ Revenu $^{\mathrm{b}}$} & Élevé & 1,0 & 1,0 & 1,0 \\
\hline & Moyen à élevé & - & $1,21(1,07-1,36)^{*}$ & $1,15(1,05-1,26)^{*}$ \\
\hline & Faible à moyen & $1,21(1,07-1,37)^{*}$ & $1,35(1,19-1,52)^{*}$ & $1,31(1,18-1,44)^{*}$ \\
\hline & Faible & $1,51(1,29-1,77)^{*}$ & $1,52(1,33-1,73)^{*}$ & $1,49(1,34-1,66)^{*}$ \\
\hline \multirow[t]{3}{*}{ Niveau de scolarité } & $\begin{array}{l}\text { Diplôme d'études } \\
\text { postsecondaires }\end{array}$ & 1,0 & 1,0 & 1,0 \\
\hline & $\begin{array}{l}\text { Diplôme d'études } \\
\text { secondaires/études } \\
\text { postsecondaires partielles }\end{array}$ & $1,21(1,04-1,33)^{*}$ & $0,87(0,80-0,94)^{*}$ & - \\
\hline & Études secondaires partielles & $1,42(1,25-1,60)^{*}$ & - & $1,13(1,05-1,22)^{*}$ \\
\hline \multirow{2}{*}{$\begin{array}{l}\text { Auto-évaluation } \\
\text { de la santé }\end{array}$} & Bonne ou excellente & 1,0 & 1,0 & 1,0 \\
\hline & Mauvaise ou passable & $2,66(2,35-3,00)^{*}$ & $2,75(2,51-3,02)^{*}$ & $2,76(2,56-2,98)^{*}$ \\
\hline \multirow[t]{2}{*}{ Maladie chronique } & Non & 1,0 & 1,0 & 1,0 \\
\hline & Oui & $2,46(2,22-2,74)^{*}$ & $1,78(1,63-1,95)^{*}$ & $2,00(1,87-2,14)^{*}$ \\
\hline \multirow[t]{2}{*}{ Usage du tabac } & Non & & 1,0 & 1,0 \\
\hline & Oui & - & $0,90(0,83-0,98)^{*}$ & $0,90(0,84-0,96)^{*}$ \\
\hline
\end{tabular}

Source : Enquête sur la santé dans les collectivités canadiennes, 2000-2001, Statistique Canada.

Abréviations : AR, agglomération de recensement; RMR, région métropolitaine de recensement; ZIM, zone d'influence métropolitaine; -, données excluses pendant la modélisation.

a Nous avons déterminé les intervalles de confiance avec 500 coefficients de pondération de bootstrap pour tenir compte du plan de sondage complexe.

b Les classes de revenus ont été établies en fonction du revenu total des ménages et du nombre de personnes par ménage.

* Statistiquement significatif à $p<0,05$. 
TABLEAU 10

Proportion (normalisée selon l'âge) de Canadiens de 15 ans et plus qui ont donné leur opinion sur la qualité des soins de santé reçus au cours des 12 derniers mois et leur niveau de satisfaction à leur égard, selon le lieu de résidence et le sexe, 2000-2001

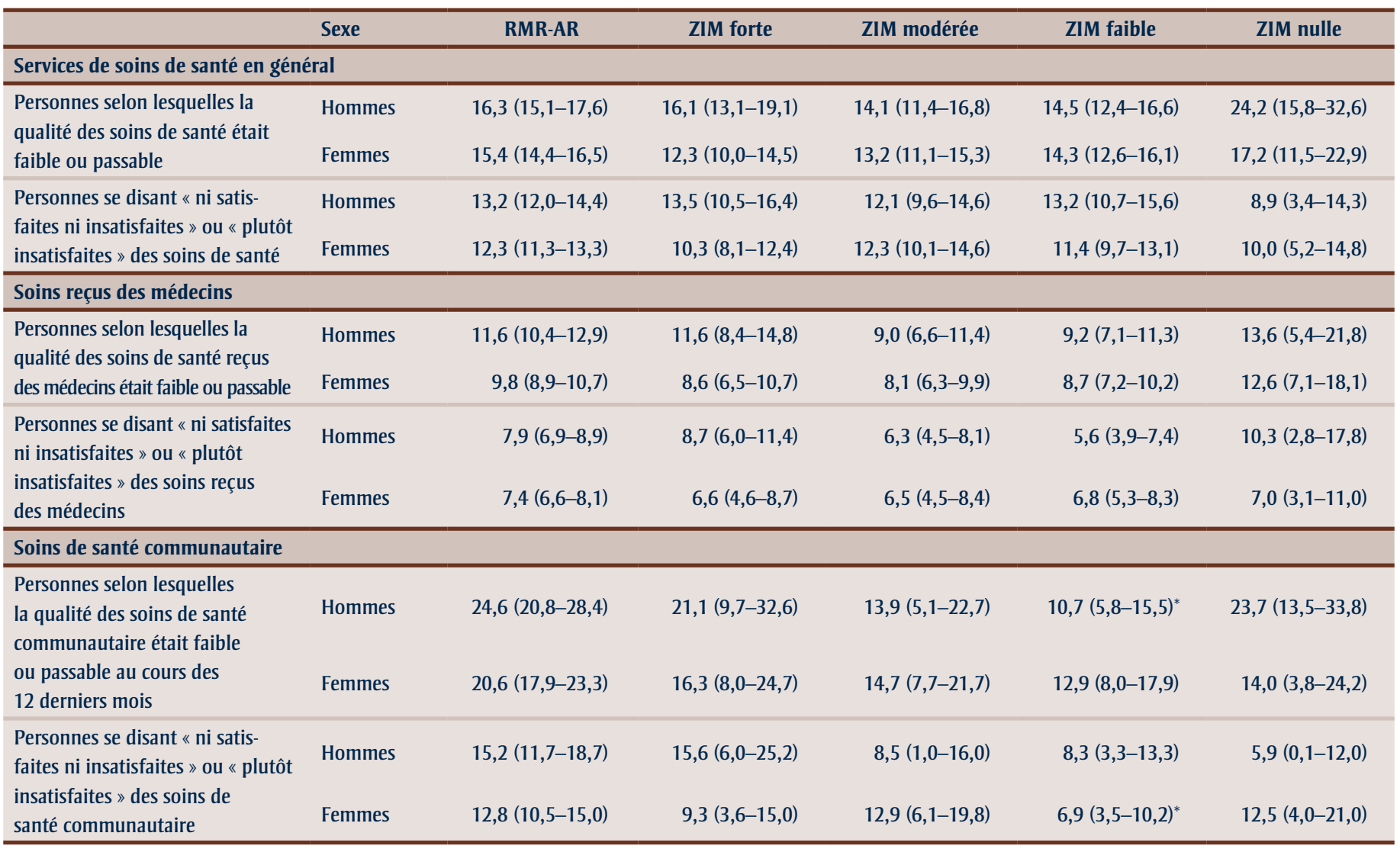

Source : Enquête sur l'accès aux services de santé 2000-2001, Statistique Canada.

Abréviations : AR, agglomération de recensement; RMR, région métropolitaine de recensement; ZIM, zone d'influence métropolitaine.

* Statistiquement significatif à $p<0,05$; le groupe de référence est la RMR-AR.

femmes de 65 à 74 ans des ZIM modérées et celles des régions urbaines (tableau 8).

L’analyse de régression multivariée des données de l'ESCC 2000-2001 a révélé que le lieu de résidence a un effet indépendant sur l'hospitalisation ayant eu lieu au cours des 12 moins précédant l'enquête pour les répondants de toutes les régions rurales, sauf les ZIM fortes. Le risque d'hospitalisation est de $10 \%$ à $27 \%$ plus élevé pour les résidents des régions rurales. Les autres facteurs de risque indépendants sont le fait d'être une femme, de ne pas avoir un revenu élevé, de ne pas être titulaire d'un diplôme d'études secondaires, d'être en mauvaise santé et de souffrir d'une maladie chronique. Les personnes de 45 ans ou plus, celles qui ne sont pas de race blanche et les fumeurs étaient tous moins à risque d'être admis à l'hôpital. La valeur du risque de certains facteurs est différente lorsque l'analyse est effectuée par sexe (tableau 9).

\section{Satisfaction à l'égard des services de santé}

Les répondants à l'ESCC 2000-2001 ont pu s'exprimer sur la qualité des soins et leur satisfaction à l'égard de trois types de services : les soins de santé en général, les soins fournis par les médecins et les soins de santé communautaire. Il n'y avait pas de différence significative sur le plan statistique entre les populations des régions urbaines et rurales quant à la perception de la qualité des soins de santé et la satisfaction à leur égard ou quant à la perception de la qualité des soins reçus des médecins et la satisfaction à l'égard de ces soins. Sur la question de la qualité des services de santé communautaire, les hommes des ZIM faibles ont été proportionnellement moins nombreux que les hommes des régions urbaines à trouver que la qualité des soins était mauvaise ou passable (RMR-AR : 24,6 \% ; ZIM faibles : 10,7 \%). Sur la même question, il n'y avait pas de différence significative sur le plan statistique entre les femmes des régions urbaines et celles des régions rurales, mais les femmes des ZIM faibles étaient, en proportion, significativement moins nombreuses que les femmes des régions urbaines à se dire insatisfaites des services de santé communautaire (RMR-AR : 12,8 \%; ZIM faibles : 6,9 \%) (tableau 10).

Ressources en santé et utilisation des services de santé à l'échelle provinciale

Les constatations de la section précédente sont fondées en grande partie sur des données autodéclarées portant sur l'ensemble du Canada. Dans la présente section, nous avons utilisé des données administratives (par exemple les données sur la facturation 
TABLEAU 11

Population selon le degré de ruralité, Nouvelle-Écosse, Ontario et Colombie-Britannique, 2001

\begin{tabular}{|c|c|c|c|c|c|c|}
\hline & \multicolumn{6}{|c|}{ Population et répartition en pourcentage (selon le découpage de 2001) } \\
\hline & \multicolumn{2}{|c|}{ Nouvelle-Écosse } & \multicolumn{2}{|c|}{ Ontario } & \multicolumn{2}{|c|}{ Colombie-Britannique } \\
\hline & Effectif & $\%$ & Effectif & $\%$ & Effectif & $\%$ \\
\hline Région urbaine (RMR-AR) & 574696 & 63,3 & 9925949 & 87,0 & 3369035 & 86,2 \\
\hline Ensemble des RRPV & 333311 & 36,7 & 1484097 & 13,0 & 538703 & 13,8 \\
\hline ZIM forte & 22209 & 2,4 & 695979 & 6,1 & 71044 & 1,8 \\
\hline ZIM modérée & 98571 & 10,9 & 489378 & 4,3 & 188811 & 4,8 \\
\hline ZIM faible & 207881 & 22,9 & 270527 & 2,4 & 236892 & 6,1 \\
\hline ZIM nulle & 4650 & 0,5 & 28213 & 0,2 & 41956 & 1,1 \\
\hline Population totale & \multicolumn{2}{|c|}{908007} & \multicolumn{2}{|c|}{11410046} & \multicolumn{2}{|c|}{3907738} \\
\hline Superficie totale & \multicolumn{2}{|c|}{55284 km² } & \multicolumn{2}{|c|}{$1076395 \mathrm{~km}^{2}$} & \multicolumn{2}{|c|}{$944735 \mathrm{~km}^{2}$} \\
\hline
\end{tabular}

Sources : Statistique Canada, recensement de la population, 2001; Ressources Naturelles Canada, Centre canadien de télédétection, Division GéoAccès, 2001.

Abréviations : AR, agglomération de recensement; RMR, région métropolitaine de recensement; ZIM, zone d’influence métropolitaine.

FIGURE 7

Taux annuels moyens de consultation des médecins de famille (normalisée selon l'âge) pour 1000 habitants, selon le lieu de résidence et le sexe, Nouvelle-Écosse, périodes de deux ans, 1997-2002

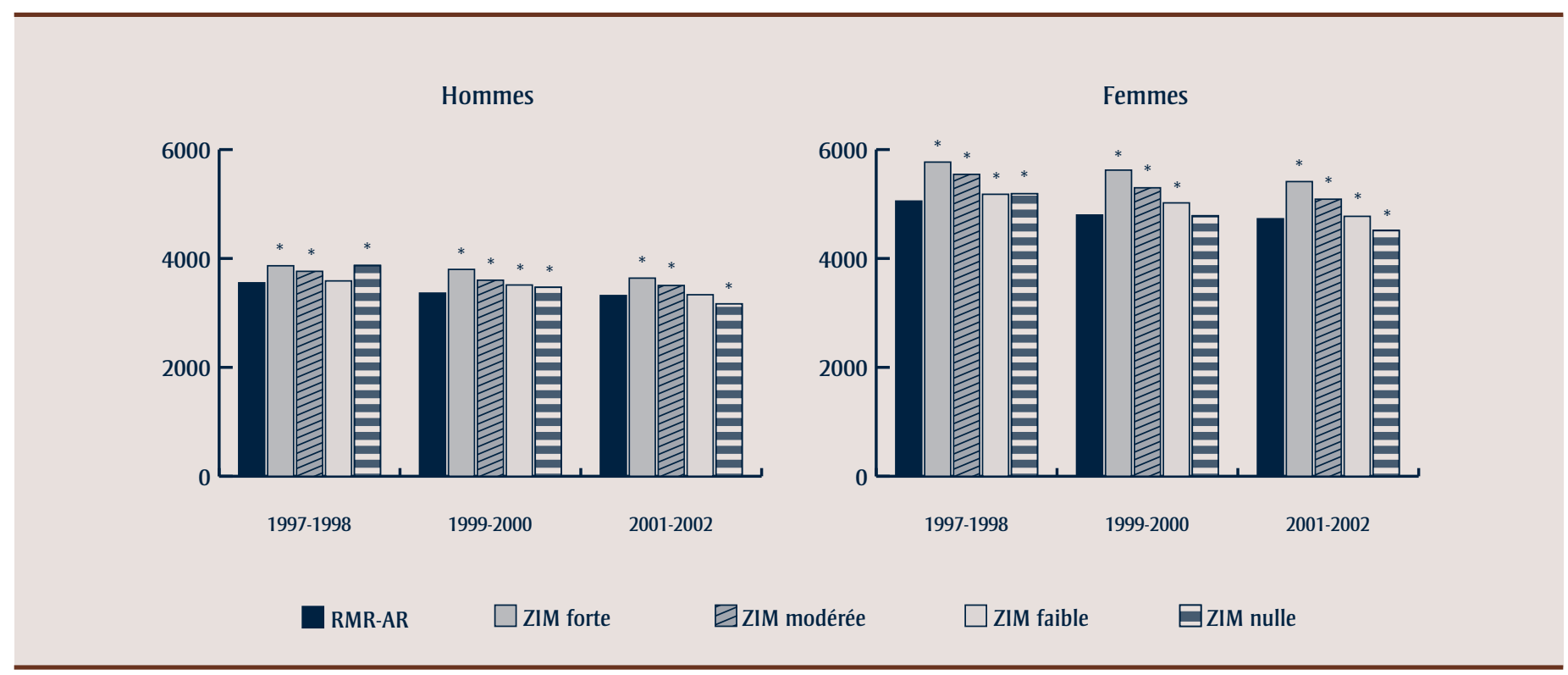

Source : Base de données sur la facturation des médecins de la Nouvelle-Écosse (Medical Services Insurance Plan), 1997-2002.

Abréviations : AR, agglomération de recensement; RMR, région métropolitaine de recensement; ZIM, zone d'influence métropolitaine.

* Statistiquement significatif à $p<0,05$; le groupe de référence est la RMR-AR. 
FIGURE 8

Taux annuels moyens de consultation des médecins spécialistes (normalisée selon l'âge) pour 1000 habitants, selon le lieu de résidence et le sexe, Nouvelle-Écosse, périodes de deux ans, 1997-2002

Hommes

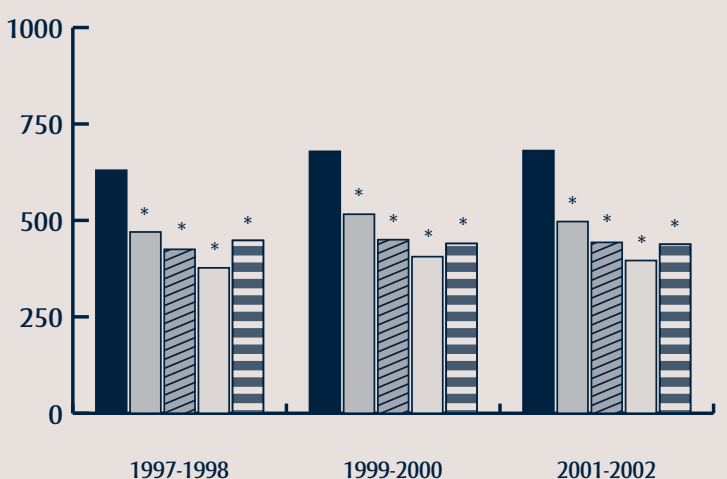

Femmes

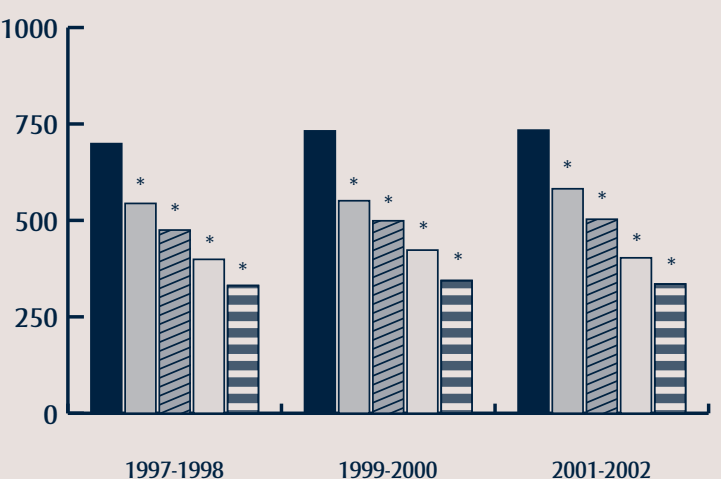

RMR-AR

ZIM forte

घzIM modérée

$\square$ ZIM faible

曰ZIM nulle

Source : Base de données sur la facturation des médecins de la Nouvelle-Écosse (Medical Services Insurance Plan), 1997-2002.

Abréviations : AR, agglomération de recensement; RMR, région métropolitaine de recensement; ZIM, zone d'influence métropolitaine.

* Statistiquement significatif à $p<0,05$; le groupe de référence est la RMR-AR.

FIGURE 9

Taux annuels moyens de consultation des chirurgiens (normalisée selon l'âge) pour 1000 habitants, selon le lieu de résidence et le sexe, Nouvelle-Écosse, périodes de deux ans, 1997-2002

Hommes

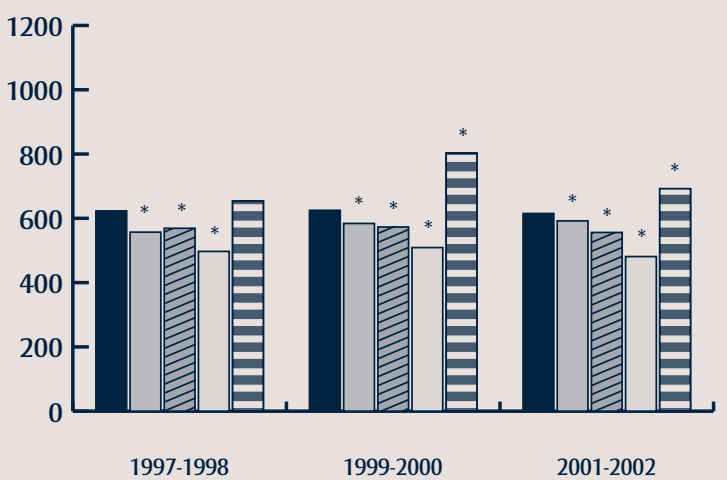

Femmes

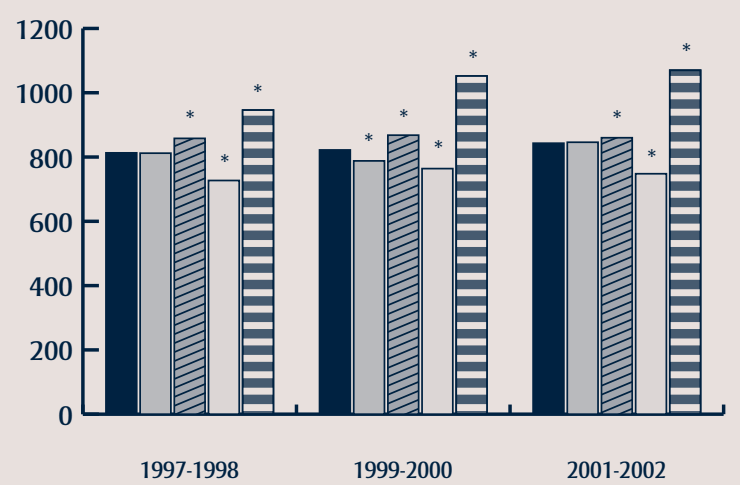

RMR-AR $\square$ ZIM forte $\quad$ ZIM modérée $\square$ ZIM faible $\quad$ ZIM nulle

Source : Base de données sur la facturation des médecins de la Nouvelle-Écosse (Medical Services Insurance Plan), 1997-2002.

Abréviations : AR, agg'lomération de recensement; RMR, région métropolitaine de recensement; ZIM, zone d'influence métropolitaine.

* Statistiquement significatif à $p<0,05$; le groupe de référence est la RMR-AR. 
des médecins) de trois provinces pour décrire l'utilisation des services de santé dans les régions rurales.

Les taux de consultation médicale et d'admission obtenus à partir des données sur la facturation des médecins et sur les congés d'hôpital ont été analysés pour la Nouvelle-Écosse, l'Ontario et la ColombieBritannique. Ces trois provinces ont été sélectionnées en fonction de la faisabilité (c'est-à-dire la disponibilité des données requises et des chercheurs ayant une expertise en santé rurale) et afin d'offrir la meilleure représentation géographique possible du pays. En Ontario et en Colombie-Britannique, la grande majorité de la population vit en région urbaine (respectivement $87 \%$ et 86,2\%); en revanche, en Nouvelle-Écosse, 63,3\% de la population vit en région urbaine et 23,4\% près du quart - dans des ZIM faibles ou nulles (contre 2,6\% en Ontario et 7,2 \% en Colombie-Britannique) (tableau 11).

\section{Nouvelle-Écosse}

Selon les données de l'ICIS, entre 1991 et 2001, seuls huit nouveaux MF se sont ajoutés aux MF de Nouvelle-Écosse et le ratio de MF à la population a diminué à la fois dans les régions rurales et les régions urbaines. En 2001, il y avait 11,6 médecins de famille pour 10000 habitants dans les RMR-AR, ce taux étant plus élevé que celui de quelque région rurale que ce soit $(7,1$ pour 10000 habitants pour l'ensemble des régions rurales). De 1997 à 2002, les résidents des ZIM fortes ont été significativement plus nombreux que ceux des régions urbaines à consulter un MF. Nous avons également observé des taux significativement plus élevés de consultation de MF chez les hommes et les femmes des autres catégories de région rurale. Ce n'est qu'au cours de la période 2001-2002 que les résidents des régions rurales ont été moins nombreux à consulter un MF, et cette observation ne s'applique qu'aux résidents des ZIM nulles (figure 7).

Le nombre de spécialistes en NouvelleÉcosse a augmenté de 139 entre 1991 et 2001 selon les données de l'ICIS. Le ratio de spécialistes dans les régions urbaines était de 13,6 pour 10000 habitants en
2001, contre 2,6 pour 10000 habitants dans l'ensemble des régions rurales. Les taux annuels de consultation des spécialistes au cours de la période de 1997-2002 étaient significativement inférieurs chez les hommes et les femmes de toutes les régions rurales et diminuaient de façon inversement proportionnelle au degré de ruralité, sauf dans le cas des hommes des ZIM nulles (figure 8). En revanche, nous avons observé des taux de consultation des chirurgiens plus faibles dans toutes les régions rurales, sauf chez les hommes des ZIM nulles en 1999-2000 et 2001-2002 et les femmes des ZIM nulles et modérées de 1997 à 2002 (figure 9).

Les taux de congé d'hôpital des NéoÉcossais, toutes causes confondues, étaient plus élevés dans toutes les régions rurales que dans les régions urbaines en 2001-2002 et augmentaient proportionnellement au degré de ruralité (RMR-AR : 89,5 pour 1000 hommes et 72,8 pour 1000 femmes; ZIM modérées : 108,4 pour 1000 hommes et 87,3 pour 1000 femmes; ZIM nulles : 232,3 pour 1000 hommes et 171,8 pour 1000 femmes). La durée des

\section{FIGURE 10}

Taux annuels moyens de consultation des médecins de famille (normalisée selon l'âge) pour 1000 habitants, selon le lieu de résidence et le sexe, Ontario, périodes de deux ans, 1997-2002

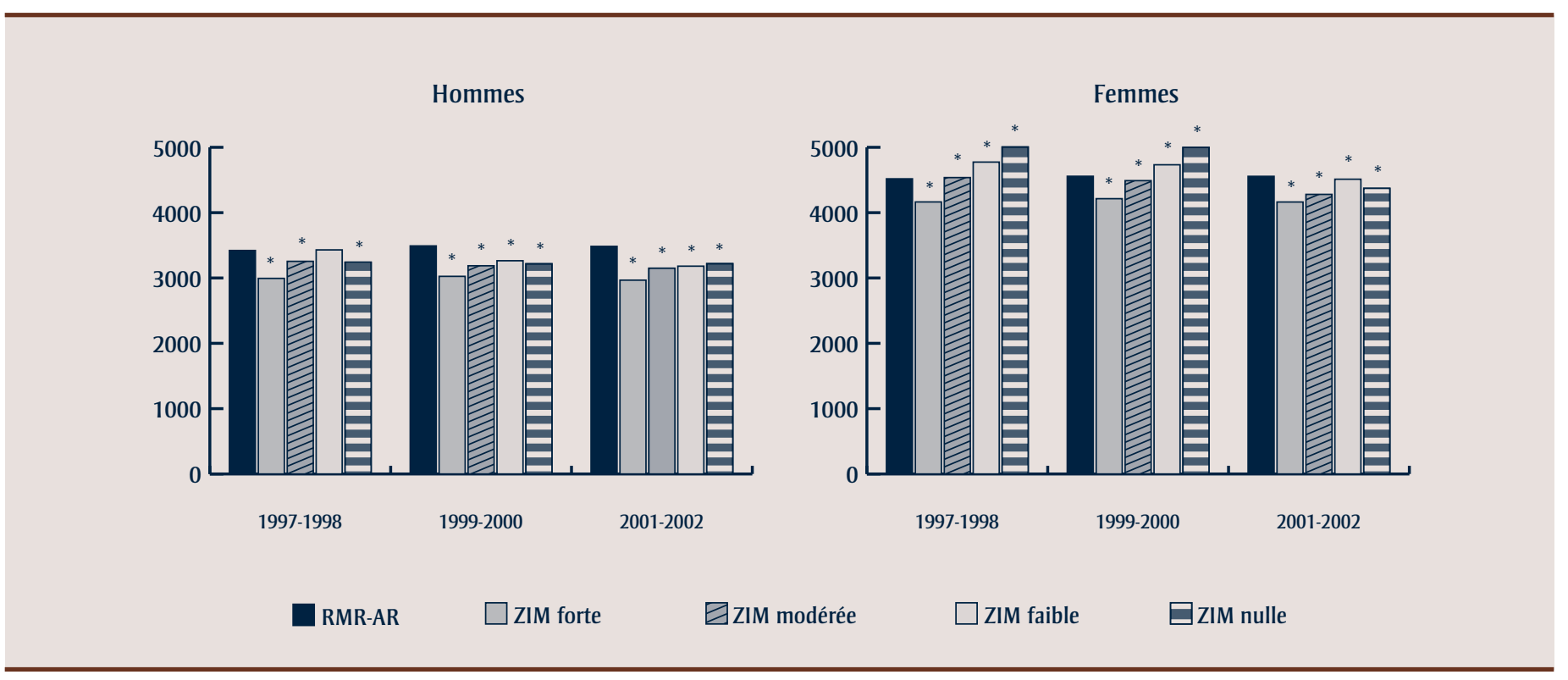

Source : Base de données sur la facturation des médecins de l'Ontario, 1997-2002.

Abréviations : AR, aggglomération de recensement; RMR, région métropolitaine de recensement; ZIM, zone d'influence métropolitaine.

Statistiquement significatif à $p<0,05$; le groupe de référence est la RMR-AR. 
FIGURE 11

Taux annuels moyens de consultation des médecins spécialistes (normalisée selon l'âge) pour 1000 habitants, selon le lieu de résidence et le sexe, Ontario, périodes de deux ans, 1997-2002

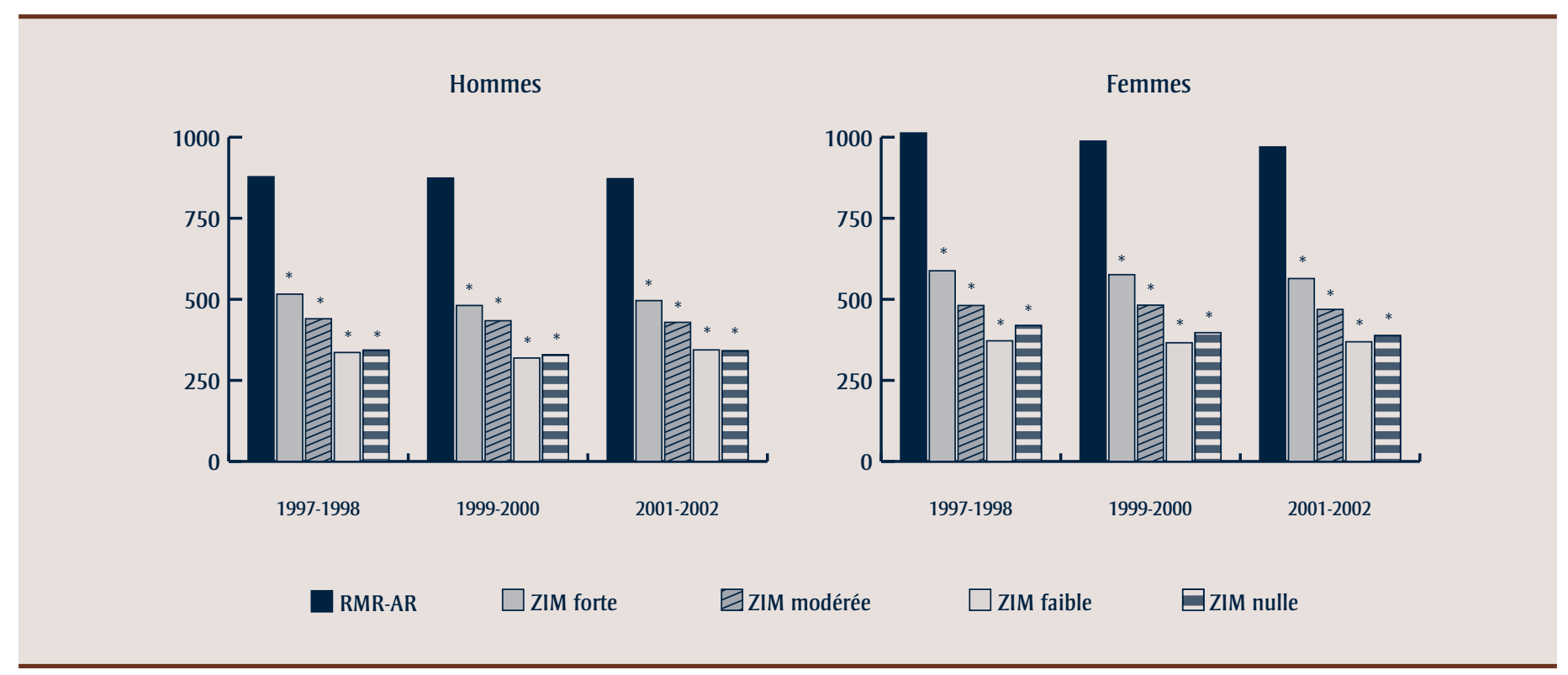

Source : Base de données sur la facturation des médecins de l'Ontario, 1997-2002.

Abréviations : AR, agglomération de recensement; RMR, région métropolitaine de recensement; ZIM, zone d'influence métropolitaine.

* Statistiquement significatif à $p<0,05$; le groupe de référence est la RMR-AR.

FIGURE 12

Taux annuels moyens de consultation des chirurgiens (normalisée selon l'âge) pour 1000 habitants, selon le lieu de résidence et le sexe, Ontario, périodes de deux ans, 1997-2002

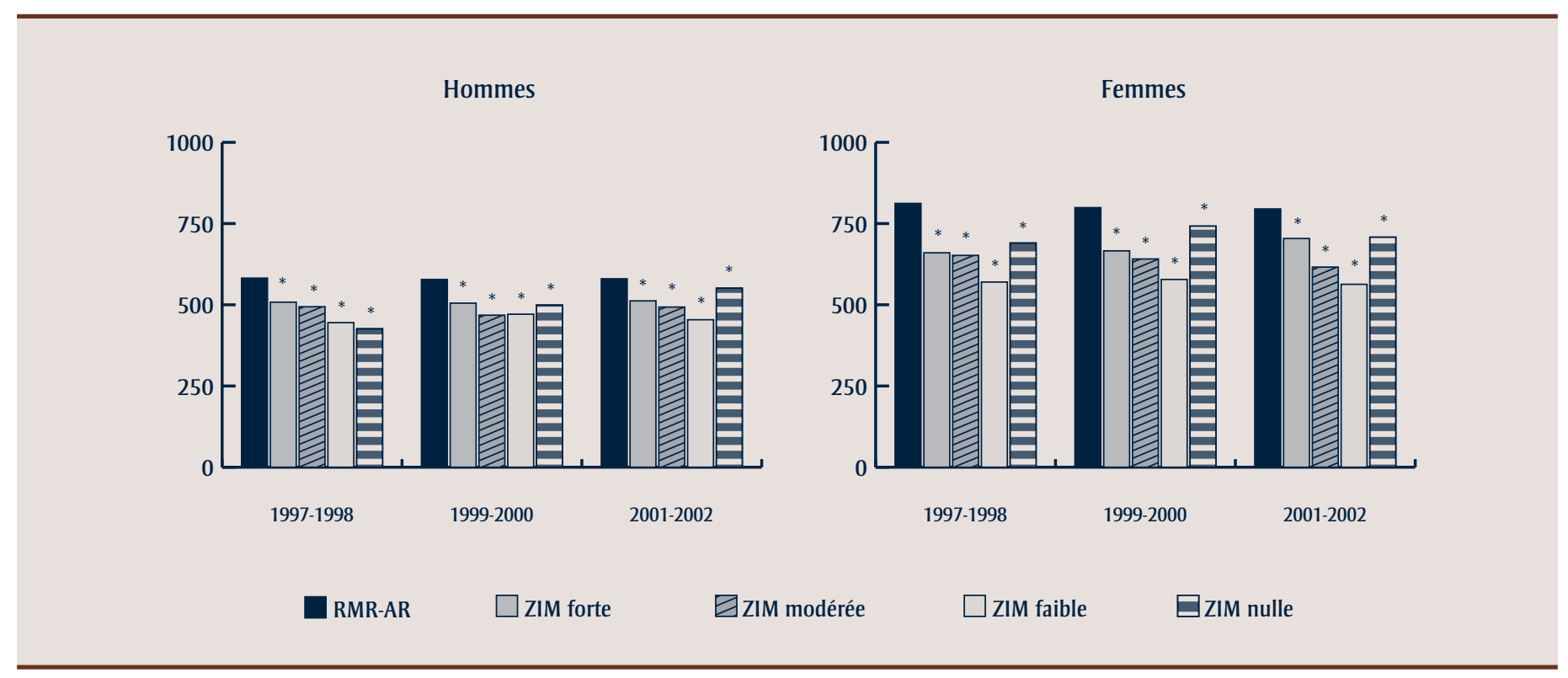

Source : Base de données sur la facturation des médecins de l'Ontario, 1997-2002.

Abréviations : AR, agglomération de recensement; RMR, région métropolitaine de recensement; ZIM, zone d'influence métropolitaine.

* Statistiquement significatif à $p<0,05$; le groupe de référence est la RMR-AR. 
hospitalisations au cours de cette période était plus courte de manière significative chez les hommes des régions rurales que chez ceux des régions urbaines $(9,01$ jours dans les RMR-AR contre 6,39 jours dans les ZIM nulles); quant aux femmes, la seule différence liée au lieu de résidence était que les femmes des ZIM nulles ont passé significativement moins de temps à l'hôpital que celles des régions urbaines $(6,92$ jours contre 9,43 jours).

\section{Ontario}

Selon les données de l'ICIS, le nombre de MF en Ontario a diminué de 370 entre 1991 et 2001 et ce recul s'est principalement fait sentir dans les régions urbaines, où le nombre de MF pour 10000 habitants est passé de 11,0 en 1991 à 9,4 en 1995 et à 9,0 en 2001. Les ratios correspondants de MF pour 10000 habitants dans l'ensemble des ZIM étaient de 5,9 en 1991, 5,5 en 1996 et 6,3 en 2001. Dans les ZIM nulles, ce ratio est passé de 2,1 à 5,5 pour 10000 habitants de 1991 à 2001.

Les taux annuels de consultation des MF pour 1000 habitants entre 1997 et 2002 étaient similaires pour les hommes entre les diverses régions rurales, mais inférieurs de façon significative à ceux des régions urbaines. En 2001-2002, les taux de consultation des MF, sans égard au sexe, étaient significativement plus faibles dans les régions rurales. Cependant, ces taux augmentaient proportionnellement au degré de ruralité chez les femmes des diverses régions rurales, sauf chez celles des ZIM nulles en 2001-2002. Sans égard au sexe, les taux de consultation des MF étaient plus faibles de manière significative dans les ZIM fortes que dans les régions urbaines durant toute la période de 1997 à 2002 (figure 10).

Entre 1991 et 2001, le nombre de spécialistes en Ontario a augmenté de 1440 selon les données de l'ICIS. Les ratios de spécialistes sont demeurés stables au fil des ans dans les régions urbaines (10,6 pour 10000 habitants en 1991, 10,2 en 1996 et 10,6 en 2001) et dans la plupart des régions rurales (ensemble des régions rurales :
0,7 pour 10000 habitants en 1991, 0,7 en 1996 et 0,9 en 2001). Sans égard au sexe, les taux de consultation des spécialistes en Ontario ont été significativement plus élevés - parfois deux fois plus élevés dans les régions urbaines que dans les régions rurales entre 1997 et 2002 et ont généralement diminué de façon inversement proportionnelle au degré de ruralité (figure 11). Bien que les taux de consultation des chirurgiens aient suivi cette tendance, ils ont été plus élevés dans les ZIM nulles que dans toute autre catégorie de ZIM, tant chez les femmes durant toute la période de 1997 à 2002 que chez les hommes au cours de la période de 2001-2002 (figure 12).

Selon la BDMH, les taux de congé d'hôpital des Ontariens, toutes causes confondues, étaient plus élevés chez les femmes que chez les hommes en 2001-2002. Ces taux étaient plus élevés chez les Ontariens de toutes les régions rurales et augmentaient proportionnellement au degré de ruralité $(66,4$ pour 1000 hommes des RMR-AR contre 128,6 pour 1000 hommes des ZIM nulles; 86,1 pour 1000 femmes des RMR-AR contre 178,8 pour 1000 femmes des ZIM nulles). En général, il n’y avait pas de différence significative sur le plan de la durée moyenne de l'hospitalisation entre les hommes des régions rurales et urbaines de l'Ontario en 2001-2002. Par ailleurs, la durée moyenne de l'hospitalisation des femmes des ZIM fortes et faibles a été inférieure à celle des femmes des régions urbaines (RMR-AR : 7,06 jours; ZIM fortes : 5,87 jours; ZIM modérée : 6,00 jours).

\section{Colombie-Britannique}

Selon les données de l'ICIS, le nombre de MF en Colombie-Britannique a augmenté de 608 entre 1991 et 2001. Dans les régions urbaines, le nombre de MF pour 10000 habitants a quelque peu diminué (de 11,6 en 1991 à 11,0 en 1996 et à 11,2 en 2001), mais était plus élevé que celui de toutes les régions rurales, sauf dans le cas des ZIM faibles (12,6 pour 10000 habitants en 2001). Durant cette période, le nombre de spécialistes a également augmenté (de 590) et leur nombre pour 10000 habitants était aussi plus élevé dans les régions urbaines que dans les régions rurales (9,6 dans les régions urbaines contre 1,6 dans l'ensemble des régions rurales) en 2001.

Sauf dans le cas des ZIM fortes, où les taux étaient significativement inférieurs à ceux des régions urbaines, les taux de congés de l'hôpital étaient plus élevés chez les résidents ruraux que chez les résidents urbains (RMR-AR : 65,4 pour 1000 hommes et 84,6 pour 1000 femmes; ZIM modérées : 70,3 pour 1000 hommes et 92,2 pour 1000 femmes; ZIM nulles : 105,6 pour 1000 hommes et 151,2 pour 1000 femmes). La durée de l'hospitalisation des hommes des ZIM modérées, faibles et nulles était significativement inférieure à celle des hommes des régions urbaines (RMR-AR : 8,04 jours; ZIM modérées : 6,58 jours; ZIM faibles : 6,48 jours; ZIM nulles : 5,79 jours). Chez les femmes, la seule différence significative sur le plan de la durée de l'hospitalisation en fonction du lieu de résidence est que les femmes des ZIM faibles étaient hospitalisées moins longtemps (6,06 jours) que les femmes des régions urbaines ( 8,00 jours).

\section{Utilisation des services selon les maladies dans trois provinces}

Les données sur les consultations médicales et sur les hospitalisations (taux normalisés selon l'âge) pour certaines maladies et catégories de maladies en Nouvelle-Écosse, en Ontario et en Colombie-Britannique en 2001-2002 ont servi à calculer les probabilités relatives d'utiliser les services de santé dans les régions rurales par rapport aux régions urbaines. Les maladies analysées étaient les maladies de la circulation, les néoplasmes, les maladies du système respiratoire, les maladies de l'appareil locomoteur et du tissu conjonctif, les troubles mentaux, les maladies du système nerveux et des organes sensoriels et le diabète. Les consultations médicales et les hospitalisations pour cause de traumatisme ou d'intoxication ont aussi été examinées. Toutes les maladies ne pouvaient être analysées dans chaque province en raison

* Pour des résultats plus détaillés, voir les tableaux en annexe 2. 
TABLEAU 12

Sommaire des risques relatifs de consultation d'un médecin et d'hospitalisation pour certaines catégories de maladies, selon le lieu de résidence et le sexe, Nouvelle-Écosse, 2001-2002

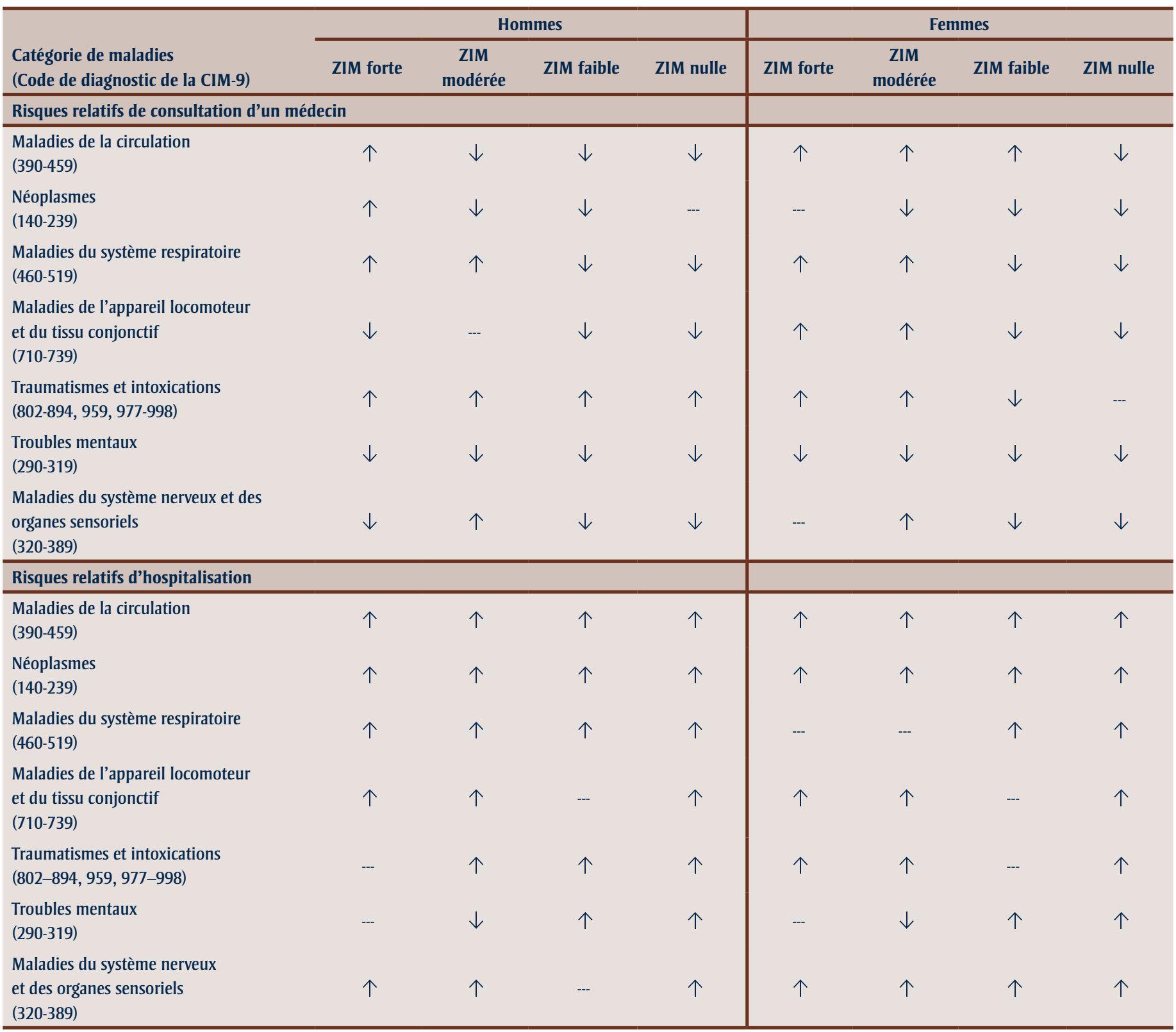

Source des données : Base de données sur la facturation des médecins de la Nouvelle-Écosse, 2001-2002.

Abréviations : AR, agglomération de recensement; RMR, région métropolitaine de recensement; CIM-9, Classification statistique internationale des maladies, traumatismes et causes de décès, neuvième édition; ZIM, zone d'influence métropolitaine.

个 risque relatif statistiquement plus élevé à $p<0,05 ; \downarrow$ risque relatif statistiquement moins élevé à $p<0,05$;--- aucune différence statistique à $p<0,05$; le groupe de référence est la RMR-AR. 
TABLEAU 13

Risques relatifs de consultation chez le médecin (normalisée selon l'âge) pour troubles mentaux, traumatismes ou intoxications, selon le lieu de résidence et le sexe, Nouvelle-Écosse, 2001-2002

\begin{tabular}{llllll}
\hline $\begin{array}{l}\text { Catégorie de maladies } \\
\text { (Code de diagnostic de la CIM-9) }\end{array}$ & Sexe & ZIM forte & ZIM modérée & ZIM faible & ZIM nulle \\
\hline Troubles mentaux & Hommes & $0,75(0,73-0,78)^{*}$ & $0,84(0,83-0,85)^{*}$ & $0,70(0,69-0,71)^{*}$ & $0,88(0,82-0,94)^{*}$ \\
$(290-319)$ & Femmes & $0,94(0,92-0,95)^{*}$ & $0,89(0,88-0,90)^{*}$ & $0,73(0,72-0,74)^{*}$ & $0,80(0,76-0,84)^{*}$ \\
\hline Traumatismes et intoxications & Hommes & $1,19(1,16-1,23)^{*}$ & $1,18(1,16-1,19)^{*}$ & $1,08(1,06-1,09)^{*}$ & $1,21(1,14-1,28)^{*}$ \\
$(802-894,959,977-998)$ & Femmes & $1,22(1,19-1,25)^{*}$ & $1,11(1,09-1,12)^{*}$ & $0,98(0,97-0,99)^{*}$ & $0,96(0,90-1,03)$ \\
\hline
\end{tabular}

Source des données : Base de données sur la facturation des médecins de la Nouvelle-Écosse, 2001-2002

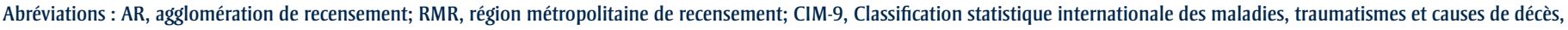
neuvième édition; ZIM, zone d'influence métropolitaine.

* Statistiquement significatif à $p<0,05$; le groupe de référence est la RMR-AR (risque $=1,00$ ).

TABLEAU 14

Risques relatifs de congé d'hôpital (normalisé selon l'âge) pour troubles mentaux, selon le lieu de résidence et le sexe, Nouvelle-Écosse, 2001-2002

\begin{tabular}{llllcc}
\hline $\begin{array}{l}\text { Catégorie de maladies } \\
\text { (Code de diaǵnostic de la CIM-9) }\end{array}$ & Sexe & ZIM forte & ZIM modérée & ZIM faible & ZIM nulle \\
\hline $\begin{array}{l}\text { Troubles mentaux } \\
(290-319)\end{array}$ & Hommes & $0,84(0,65-1,11)$ & $0,81(0,68-0,96)^{*}$ & $1,37(1,23-1,53)^{*}$ & $3,69(2,58-5,29)^{*}$ \\
\hline
\end{tabular}

Source des données : Base de données sur la morbidité hospitalière, 2001-2002, Institut canadien d'information sur la santé.

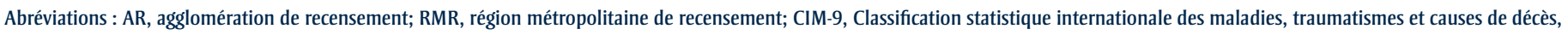
neuvième édition; ZIM, zone d'influence métropolitaine.

* Statistiquement significatif à $p<0,05$; le groupe de référence est la RMR-AR (risque = 1,00).

de problèmes d'extraction des données, notamment en Colombie-Britannique où l'analyse des consultations chez le médecin portait plus sur des maladies précises que sur des grandes catégories de maladies*.

\section{Nouvelle-Écosse}

En Nouvelle-Écosse, les risques relatifs de consultation d'un médecin variaient beaucoup mais, en général, ces risques étaient plus élevés dans les ZIM fortes et moins élevés dans les ZIM faibles et les ZIM nulles. Les tendances dans les ZIM modérées dépendaient beaucoup des sept catégories analysées (6 catégories de maladies ainsi que la catégorie traumatismes et intoxications) (tableau 12).

Les Néo-Écossais ruraux affichaient systématiquement des risques de consultation chez un médecin moins élevés que les Néo-Écossais urbains pour des troubles mentaux, mais systématiquement plus élevés pour cause de traumatisme ou d'intoxication, à l'exception des femmes des ZIM faibles et des ZIM nulles (tableau 13).

En ce qui concerne les taux de congé d'hôpital, les Néo-Écossais ruraux affichaient des risques relatifs plus élevés que les Néo-Écossais urbains, sauf pour les troubles mentaux où les résultats variaient : ils étaient beaucoup plus élevés dans les ZIM faibles et les ZIM nulles, mais moins élevés dans les ZIM modérées et les ZIM fortes (tableau 14).

\section{Ontario}

Les risques de consultation médicale et d'hospitalisation étaient relativement constants : les résidents ruraux, sans égard au sexe, étaient en général exposés à de moins grands risques de consulter un médecin que les résidents urbains, même s'ils étaient exposés à de plus grands risques d'être hospitalisés pour les mêmes catégories de maladies et pour des traumatismes ou des intoxications (tableau 15). Toutefois, comparativement aux résidents urbains, les Ontariens ruraux tant hommes que femmes étaient exposés à de plus grands risques de consulter leur médecin pour des traumatismes ou des intoxications sauf dans le cas des hommes et des femmes dans les ZIM fortes (tableau 16).

Les risques de congé d'hôpital étaient clairement plus élevés chez les résidents ruraux, hommes ou femmes, que chez les résidents urbains, pour toutes les catégories de maladies, à l'exception des troubles mentaux chez les résidents tant hommes que femmes des ZIM fortes (tableau 17).

\section{Colombie-Britannique}

En général, les consultations auprès d'un médecin étaient proportionnellement beaucoup moins nombreuses chez les résidents des ZIM fortes et faibles que chez les résidents urbains pour la plupart des maladies examinées. Toutefois, les résidents des ZIM nulles étaient proportionnellement plus nombreux à consulter un médecin pour les maladies cardio-vasculaires, 
TABLEAU 15

Risques relatifs de consultation d'un médecin et d'hospitalisation pour certaines catégories de maladies, selon le lieu de résidence et le sexe, Ontario, 2001-2002

\begin{tabular}{|c|c|c|c|c|c|c|c|c|}
\hline \multirow[b]{2}{*}{$\begin{array}{l}\text { Catégorie de maladies } \\
\text { (Code de diagnostic de la CIM-9) }\end{array}$} & \multicolumn{4}{|c|}{ Hommes } & \multicolumn{4}{|c|}{ Femmes } \\
\hline & ZIM forte & $\begin{array}{l}\text { ZIM } \\
\text { modérée }\end{array}$ & ZIM faible & ZIM nulle & ZIM forte & $\begin{array}{l}\text { ZIM } \\
\text { modérée }\end{array}$ & ZIM faible & ZIM nulle \\
\hline $\begin{array}{l}\text { Maladies de la circulation } \\
(390-459)\end{array}$ & $\downarrow$ & $\downarrow$ & $\downarrow$ & --- & $\uparrow$ & $\downarrow$ & $\downarrow$ & $\downarrow$ \\
\hline $\begin{array}{l}\text { Néoplasmes } \\
(140-239)\end{array}$ & $\downarrow$ & $\downarrow$ & $\downarrow$ & --- & $\downarrow$ & $\downarrow$ & $\downarrow$ & $\uparrow$ \\
\hline $\begin{array}{l}\text { Maladies du système respiratoire } \\
(460-519)\end{array}$ & $\downarrow$ & $\downarrow$ & $\downarrow$ & $\downarrow$ & $\downarrow$ & $\downarrow$ & $\downarrow$ & $\downarrow$ \\
\hline $\begin{array}{l}\text { Maladies de l'appareil locomoteur } \\
\text { et du tissu conjonctif } \\
\text { (710-739) }\end{array}$ & $\downarrow$ & --- & $\downarrow$ & $\downarrow$ & $\downarrow$ & $\downarrow$ & $\downarrow$ & --- \\
\hline $\begin{array}{l}\text { Maladies du système nerveux et des } \\
\text { organes sensoriels } \\
\left(320-389^{*}\right)\end{array}$ & $\downarrow$ & $\downarrow$ & $\downarrow$ & $\downarrow$ & $\downarrow$ & $\downarrow$ & $\downarrow$ & $\downarrow$ \\
\hline \multicolumn{9}{|l|}{ Risques relatifs d'hospitalisation } \\
\hline $\begin{array}{l}\text { Maladies de la circulation } \\
(390-459)\end{array}$ & $\uparrow$ & $\uparrow$ & $\uparrow$ & $\uparrow$ & $\uparrow$ & $\uparrow$ & $\uparrow$ & $\uparrow$ \\
\hline $\begin{array}{l}\text { Néoplasmes } \\
(140-239)\end{array}$ & -- & $\uparrow$ & $\uparrow$ & $\uparrow$ & $\uparrow$ & $\uparrow$ & $\uparrow$ & $\uparrow$ \\
\hline $\begin{array}{l}\text { Maladies du système respiratoire } \\
\left(460-519^{*}\right)\end{array}$ & $\uparrow$ & $\uparrow$ & $\uparrow$ & $\uparrow$ & $\uparrow$ & $\uparrow$ & $\uparrow$ & $\uparrow$ \\
\hline $\begin{array}{l}\text { Maladies du système nerveux et des } \\
\text { organes sensoriels } \\
(320-389)\end{array}$ & --- & $\uparrow$ & $\uparrow$ & $\uparrow$ & $\uparrow$ & $\uparrow$ & $\uparrow$ & $\uparrow$ \\
\hline
\end{tabular}

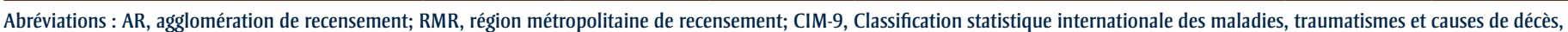
neuvième édition; ZIM, zone d'influence métropolitaine.

Le groupe de référence est la RMR-AR (risque $=1,00$ ); $\uparrow$ risque relatif statistiquement plus élevé à $p<0,05 ; \downarrow$ risque relatif statistiquement moins élevé à $p<0,05$;

--- aucune différence statistique à $p<0,05$. 
TABLEAU 16

Risques relatifs de consultation d'un médecin (normalisée selon l'âge) pour traumatismes et intoxications, selon le lieu de résidence et le sexe, Ontario, 2001-2002

\begin{tabular}{|c|c|c|c|c|c|}
\hline $\begin{array}{l}\text { Catégorie de maladies } \\
\text { (Code de diagnostic de la CIM-9) }\end{array}$ & Sexe & ZIM forte & ZIM modérée & ZIM faible & ZIM nulle \\
\hline \multirow{2}{*}{$\begin{array}{l}\text { Traumatismes et intoxications } \\
(802-894,959,977-998)\end{array}$} & Hommes & $0,98(0,98-0,99)^{*}$ & $1,10(1,09-1,10)^{*}$ & $1,15(1,14-1,16)^{*}$ & $1,17(1,15-1,20)^{*}$ \\
\hline & Femmes & $0,94(0,94-0,95)^{*}$ & $1,01(1,00-1,02)^{*}$ & $1,06(1,05-1,07)^{*}$ & $1,23(1,20-1,26)^{*}$ \\
\hline
\end{tabular}

Source des données : Base de données sur la facturation des médecins de l’Ontario, 2001-2002.

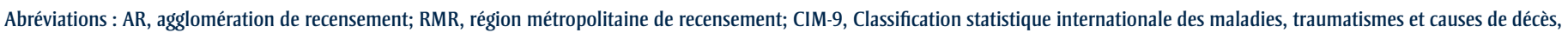
neuvième édition; ZIM, zone d'influence métropolitaine.

* Statistiquement significatif à $p<0,05$; le groupe de référence est la RMR-AR (risque $=1,00$ ).

TABLEAU 17

Risques relatifs de congé d'hôpital (normalisé selon l'âge) pour troubles mentaux, selon le lieu de résidence et le sexe, Ontario, $2001-2002$

\begin{tabular}{llllll}
\hline $\begin{array}{l}\text { Catégorie de maladies } \\
\text { (Code de diagnostic de la CIM-9) }\end{array}$ & Sexe & ZIM forte & ZIM modérée & ZIM faible \\
\hline $\begin{array}{lllll}\text { Troubles mentaux } \\
(290-319)\end{array}$ & Hommes & $0,82(0,75-0,90)^{*}$ & $1,54(1,46-1,61)^{*}$ & $1,99(1,92-2,07)^{*}$ & $2,79(2,47-3,15)^{*}$ \\
\hline
\end{tabular}

Source des données : Base de données sur la morbidité hospitalière, 2001-2002, Institut canadien d'information sur la santé.

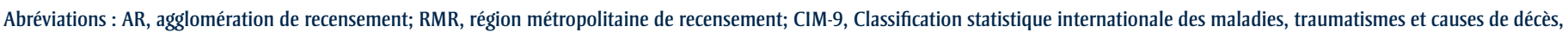
neuvième édition; ZIM, zone d'influence métropolitaine.

* Statistiquement significatif à $p<0,05$; le groupe de référence est la RMR-AR (risque $=1,00$ ).

les néoplasmes du sein, les néoplasmes du poumon, les maladies pulmonaires obstructives chroniques, l'arthrite et le diabète (tableau 18). Les résidents des ZIM faibles et nulles étaient exposés à des risques d'hospitalisation beaucoup plus grands que les résidents urbains et, pour un certain nombre de maladies, cela s'appliquait aussi aux résidents des ZIM modérées. Dans les ZIM fortes, les risques d'hospitalisation étaient plus faibles ou les mêmes que dans les zones urbaines.

\section{Utilisation des services de santé par ZIM}

L'utilisation des services de santé en Nouvelle-Écosse, en Ontario et en ColombieBritannique peut aussi être présentée sous l'angle des similitudes et des différences selon les degrés de ruralité en lien avec les risques relatifs de consultation d'un médecin ou d'hospitalisation.

\section{ZIM fortes}

- Les Néo-Écossais qui vivent dans les ZIM fortes étaient exposés à des risques relatifs de consultation chez le médecin (sauf pour les troubles mentaux et les maladies de l'appareil locomoteur et du tissu conjonctif et les maladies du système nerveux) égaux ou supérieurs et à des risques d'hospitalisation égaux ou supérieurs aux Néo-Écossais urbains pour toutes les maladies examinées.

- Les Ontariens qui vivent dans les ZIM fortes étaient en général exposés à des risques relatifs de consultation chez le médecin moins élevés mais à des risques d'hospitalisation plus élevés que les Ontariens urbains. Les femmes des ZIM fortes, qui affichaient des risques de consultation chez le médecin plus élevés pour les maladies de la circulation, constituaient une des rares exceptions. Les risques d'hospitalisation étaient supérieurs pour toutes les catégories de maladies à l'exception des troubles mentaux. De même, aucune différence statistiquement significative n'a été observée chez les hommes atteints de cancers, de maladies du système nerveux ou de maladies des organes sensoriels.

- Les Britanno-Colombiens vivant dans les ZIM fortes affichaient systématiquement des risques de consultation chez le médecin inférieurs ou égaux pour les onze maladies examinées, comparativement aux Britanno-Colombiens urbains, sauf dans le cas des femmes atteintes d'arthrite rhumatoïde ou de la maladie d'Alzheimer. Les risques d'hospitalisation étaient en général égaux ou inférieurs à ceux des régions urbaines, sauf chez les femmes atteintes de maladie de la circulation, qui présentaient des risques plus élevés.

ZIM modérées et ZIM faibles

- Les Néo-Écossais et les BritannoColombiens des ZIM modérées et faibles affichaient des risques de consultation chez le médecin qui n'étaient pas constants. Des différences ont été observées entre les hommes et les femmes et les risques relatifs variaient d'une catégorie de maladies à une autre (Nouvelle-Écosse) ou d'une maladie à une autre (Colombie-Britannique). Toutefois, les Ontariens des ZIM modérées et des ZIM faibles étaient en général exposés à des risques de consultation chez le médecin moins 
TABLEAU 18

Risques relatifs de consultation d'un médecin et d'hospitalisation pour certaines maladies, selon le lieu de résidence et le sexe, Colombie Britannique, 2001-2002

\begin{tabular}{|c|c|c|c|c|c|c|c|c|}
\hline \multirow[b]{2}{*}{$\begin{array}{l}\text { Catégorie de maladies } \\
\text { (Code de diagnostic de la CIM-9) }\end{array}$} & \multicolumn{4}{|c|}{ Hommes } & \multicolumn{4}{|c|}{ Femmes } \\
\hline & ZIM forte & $\begin{array}{l}\text { ZIM } \\
\text { modérée }\end{array}$ & ZIM faible & ZIM nulle & ZIM forte & $\begin{array}{l}\text { ZIM } \\
\text { modérée }\end{array}$ & ZIM faible & ZIM nulle \\
\hline $\begin{array}{l}\text { Maladie ischémique cardiaque } \\
(410-414)\end{array}$ & $\downarrow$ & $\downarrow$ & $\downarrow$ & $\downarrow$ & $\downarrow$ & $\downarrow$ & $\downarrow$ & --- \\
\hline $\begin{array}{l}\text { Maladie cérébro-vasculaire } \\
(430-434)\end{array}$ & $\downarrow$ & $\downarrow$ & $\downarrow$ & $\uparrow$ & --- & $\downarrow$ & $\downarrow$ & $\uparrow$ \\
\hline $\begin{array}{l}\text { Néoplasme du sein } \\
\text { (174) }\end{array}$ & S.o. & s.o. & s.o. & S.o. & $\downarrow$ & $\uparrow$ & $\uparrow$ & $\uparrow$ \\
\hline $\begin{array}{l}\text { Néoplasme du poumon } \\
\text { (162) }\end{array}$ & --- & $\uparrow$ & $\uparrow$ & $\uparrow$ & --- & $\uparrow$ & --- & $\uparrow$ \\
\hline $\begin{array}{l}\text { Asthme } \\
(493)\end{array}$ & $\downarrow$ & $\downarrow$ & $\downarrow$ & $\downarrow$ & $\downarrow$ & $\downarrow$ & $\downarrow$ & --- \\
\hline $\begin{array}{l}\text { Arthrite rhumatoïde } \\
\text { (714) }\end{array}$ & -- & $\uparrow$ & $\downarrow$ & $\uparrow$ & $\uparrow$ & $\uparrow$ & $\uparrow$ & $\uparrow$ \\
\hline $\begin{array}{l}\text { Ostéoarthrite } \\
\text { (715) }\end{array}$ & $\downarrow$ & $\uparrow$ & $\downarrow$ & $\uparrow$ & $\downarrow$ & $\uparrow$ & $\downarrow$ & $\uparrow$ \\
\hline $\begin{array}{l}\text { Dépression } \\
(296.2,296.3,300.4,311)\end{array}$ & $\downarrow$ & $\downarrow$ & $\downarrow$ & $\downarrow$ & $\downarrow$ & $\downarrow$ & $\downarrow$ & $\downarrow$ \\
\hline $\begin{array}{l}\text { Maladie d'Alzheimer/troubles démentiels } \\
\text { (331) }\end{array}$ & $\downarrow$ & $\uparrow$ & $\downarrow$ & --- & $\uparrow$ & $\uparrow$ & $\downarrow$ & $\downarrow$ \\
\hline $\begin{array}{l}\text { Diabète } \\
(250)\end{array}$ & $\downarrow$ & $\downarrow$ & $\downarrow$ & $\uparrow$ & $\downarrow$ & $\downarrow$ & $\downarrow$ & $\uparrow$ \\
\hline \multicolumn{9}{|l|}{ Risques relatifs d'hospitalisation } \\
\hline $\begin{array}{l}\text { Maladies de l'appareil locomoteur } \\
\text { et du tissu conjonctif } \\
\text { (710-739) }\end{array}$ & --- & $\uparrow$ & $\uparrow$ & $\uparrow$ & --- & $\uparrow$ & $\uparrow$ & $\uparrow$ \\
\hline $\begin{array}{l}\text { Traumatismes et intoxications } \\
(802-894,959,977-998)\end{array}$ & --- & $\uparrow$ & $\uparrow$ & $\uparrow$ & --- & $\uparrow$ & $\uparrow$ & $\uparrow$ \\
\hline $\begin{array}{l}\text { Troubles mentaux } \\
(290-319)\end{array}$ & $\downarrow$ & $\uparrow$ & $\uparrow$ & $\uparrow$ & $\downarrow$ & --- & $\uparrow$ & $\uparrow$ \\
\hline $\begin{array}{l}\text { Maladies du système nerveux et des } \\
\text { organes sensoriels } \\
(320-389)\end{array}$ & $\downarrow$ & --- & $\uparrow$ & $\uparrow$ & --- & --- & $\uparrow$ & $\uparrow$ \\
\hline
\end{tabular}

Source des données : Base de données sur la facturation des médecins de la Colombie-Britannique, 2001-2002.

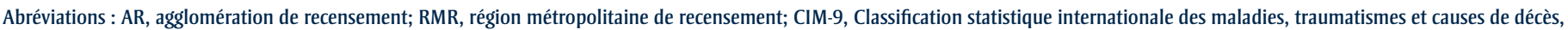
neuvième édition; ZIM, zone d'influence métropolitaine.

Le groupe de référence est la RMR-AR (risque $=1,00$ ); $\uparrow$ risque relatif statistiquement plus élevé à $p<0,05 ; \downarrow$ risque relatif statistiquement moins élevé à $p<0,05$;

--- aucune différence statistique à $p<0,05$. 
élevés, à l'exception de la catégorie traumatismes et intoxications, tant chez les hommes que chez les femmes.

- Par contre, les résidents des ZIM modérées et des ZIM faibles des trois provinces affichaient en général des risques d'hospitalisation plus élevés. Les quelques exceptions ont surtout été observées dans les ZIM modérées de la Colombie-Britannique, chez les hommes et chez les femmes, et dans les ZIM modérées et les ZIM faibles de la Nouvelle-Écosse, surtout chez les femmes.

\section{ZIM nulles}

- De manière générale, les ZIM nulles n'affichaient pas de risques différents de ceux observés dans les autres types de régions rurales. Les résidents des ZIM nulles de Nouvelle-Écosse étaient exposés à des risques de consultation chez le médecin (à l'exception de la catégorie traumatismes et intoxications chez les hommes) inférieurs ou égaux aux résidents des RMR ou des $A R$, mais à des risques d'hospitalisation plus élevés pour l'ensemble des maladies examinées.

- Les Ontariens vivant dans les ZIM nulles affichaient des risques relatifs de consultation chez le médecin inférieurs ou égaux aux Ontariens urbains, sauf dans la catégorie traumatismes et intoxications chez les hommes et chez les femmes et dans la catégorie néoplasmes chez les femmes. Toutefois, les risques relatifs d'hospitalisation étaient plus élevés dans l'ensemble des catégories de maladies tant chez les hommes que chez les femmes.

- Il ne se dégage pas de tendance nette chez les résidents des ZIM nulles de Colombie-Britannique en ce qui concerne les risques relatifs de consultation chez le médecin. Toutefois, les risques relatifs d'hospitalisation étaient systématiquement plus élevés que dans les régions urbaines. Ces résultats cadrent avec les risques d'hospitalisation plus élevés dans les ZIM faibles de Colombie-Britannique observés pour l'ensemble des maladies examinées.

\section{Analyse et conclusion}

Nous avons présenté dans les sections précédentes des données concernant les consultations de médecins et l'utilisation des services hospitaliers et d'autres services de santé par les Canadiens vivant dans des collectivités urbaines et dans différents contextes ruraux. Nous allons maintenant analyser ces résultats et leurs répercussions à la lumière de trois interrogations :

- Que signifient ces résultats sous l'angle de la santé rurale?

- Les résultats offrent-ils un éclairage neuf sur les liens entre le lieu de résidence et la santé, l'équité en matière de santé et la relation entre le système de santé canadien et les soins de santé ruraux?

- Quelles sont les incidences de ces résultats sur les recherches futures dans le domaine de la santé rurale?

Nous nous sommes fondés sur de multiples sources d'information, dont des données d'enquête et des données administratives tirées de différentes sources, pour examiner l'utilisation des services de santé des Canadiens ruraux. Nous avons enquêté sur la relation possible entre le lieu de résidence et l'utilisation des services de santé aux échelles nationale et provinciales à l'aide de données nationales et de données provinciales provenant de la Nouvelle-Écosse, de l'Ontario et de la Colombie-Britannique, en nous assurant que les résultats n'étaient pas le fait d'un travers méthodologique attribuable à une source de données d'une compétence territoriale donnée.

La quantité des données crée inévitablement des variations entre les résultats selon la base de données utilisée, la compétence territoriale, la catégorie de maladies, les services de santé et la population à l'étude. Toutefois, un certain nombre de grandes tendances se sont dégagées et semblent constantes. Par ailleurs, nous avons évité de considérer le Canada rural comme une entité homogène. De nombreux chercheurs et de nombreux planificateurs des soins de santé ont examiné les différences entre les régions rurales et les régions urbaines, mais peu se sont arrêtés à l'hétérogénéité du Canada rural du point de vue des différentes utilisations des services de santé. Pour corriger cette situation, nous avons " désagrégé » le Canada rural en catégories plus fines, en fonction du degré de ruralité et de l'éloignement des centres urbains.

La présentation synthétique des résultats a fait ressortir certaines tendances générales de l'utilisation des services de santé en milieu rural. Au premier coup d'œil, les relations entre le lieu de résidence et l'utilisation des services de santé semblent varier beaucoup et refléter des situations spécifiques, des préférences individuelles de patients, des styles de pratiques médicales différents ou des profils de prestation de services variant selon les maladies. Mais au niveau national comme au niveau provincial (au moins dans les trois provinces étudiées dans cette étude) et par rapport aux grandes catégories de maladies, certains profils se dégagent de l'utilisation des services : les résidents ruraux consultent en général moins souvent les médecins que les résidents urbains, mais affichent en revanche des risques relatifs d'hospitalisation plus élevés. Ces tendances sont particulièrement nettes chez les personnes qui vivent dans les ZIM faibles et les ZIM nulles. Il y a quelques exceptions en Colombie-Britannique, qui tiennent peut-être au fait que l'analyse des consultations médicales dans cette province portait surtout sur des maladies précises, tandis que l'analyse faite en Nouvelle-Écosse et en Ontario était axée sur des grandes catégories de maladies.

Nous allons maintenant analyser la signification et l'incidence des résultats obtenus.

\section{La « ruralité » n'est pas un concept monolithique}

Les régions rurales n'utilisent pas toutes les services de santé de la même manière. Parfois, les différences entre les types de régions rurales sont plus grandes qu'entre les collectivités rurales et les collectivités urbaines. Par exemple, les ZIM fortes semblent différer des autres régions rurales quant à l'état de santé et à l'utilisation des services de santé. Les résidents des ZIM fortes ont en général une longue espérance 
de vie (hommes : 77,4 ans; femmes : 81,5 ans) et une longue espérance de vie corrigée en fonction de la santé (hommes : 68,7 ans; femmes : 71,3 ans) ${ }^{1}$ comparativement à la population canadienne en général. Cette situation pourrait se traduire par des risques relatifs de consultation chez le médecin moins grands. Du point de vue de l'accès aux services de santé, les résidents des ZIM fortes ont peut-être moins besoin de soins de santé, se heurtent à moins d'obstacles en termes d'accessibilité ou sont mieux en mesure de surmonter les obstacles éventuels.

Inversement, et peut-être est-ce tout naturel, ce sont les résidents des ZIM nulles qui ont en général à leur disposition le moins de ressources en santé et le plus de difficultés à se prévaloir de services de santé. Pourtant, les résidents de ces régions sont en général en moins bonne condition physique. Ils ont l'espérance de vie la plus courte (hommes : 74,0 ans; femmes : 81,4 ans) et l'espérance de vie corrigée en fonction de la santé la plus courte (hommes : 65,5 ans; femmes : 69,9 ans) ${ }^{1}$. Du point de vue de la planification des soins de santé, les Canadiens des ZIM nulles (et, dans une certaine mesure, ceux des ZIM faibles) ont sans doute le plus besoin d'aide pour surmonter les difficultés d'accès aux soins et pour améliorer leur état de santé. Comme la Commission Romanow l'a souligné24 :

En fait, on pourrait dire que l'offre de soins est régie par une "loi inverse". Les habitants des collectivités rurales ont une moins bonne santé et des besoins en soins de santé primaires plus grands que les habitants des centres urbains, mais ils sont moins bien desservis et ils ont plus de mal à avoir accès aux services de santé. (p. 178)

\section{Utilisation différenciée des services}

L'accès aux services de santé et l'utilisation des services de santé diffèrent grandement entre les collectivités urbaines et les collectivités rurales. Par exemple, plus le degré de ruralité augmente, plus les taux d'hospitalisation augmentent, mais plus la durée moyenne des séjours à l'hôpital diminue. De plus, les ruraux font état en plus grande proportion de soins en services d'urgence ou dans des cliniques externes. Ces résultats témoignent non seulement de différences sur le plan de l'état de santé ou des besoins en soins de santé mais peut-être aussi de différences dans la manière dont les services de santé sont organisés ou fournis dans les régions non urbaines, ou de disparités quant à la disponibilité des ressources en santé, y compris les ressources humaines.

Par exemple, Pong et Pitblado ${ }^{3}$ ont décrit les différences entre les pratiques des médecins de famille en milieu rural et des médecins de famille en milieu urbain. Les médecins de famille en milieu rural ont en général un champ d'activité beaucoup plus étendu, ils sont davantage susceptibles de travailler dans des types variés d'établissements de soins de santé et ils sont proportionnellement plus nombreux à assurer des services cliniques et à effectuer des traitements qui seraient généralement fournis par des spécialistes dans les grands centres urbains. En l'absence relative de spécialistes dans les régions rurales, la dépendance plus grande des résidents ruraux à l'égard des soins hospitaliers et des services d'urgence pourrait être attribuable au manque d'établissements de soins ambulatoires communautaires, comme les cliniques sans rendez-vous et les centres de santé communautaire.

Les consultations auprès d'une infirmière sont plus fréquentes chez les résidents ruraux. Les habitants des ZIM modérées, faibles et nulles sont proportionnellement plus nombreux que les gens de la ville à consulter une infirmière et ceux des ZIM nulles encore plus. Les collectivités éloignées ou les très petites collectivités sont dotées de postes d'infirmières itinérantes ou d’infirmières praticiennes qui offrent un large éventail de services de santé, dont le diagnostic et le traitement de maladies mineures, les médecins assurant le suivi et la consultation à distance ou par consultations de proximité périodiques.

Ces divergences d'utilisation ne sont pas une aberration, elles sont au contraire prévisibles. Il importe de faire la distinction entre les variations régionales dans l'utilisation des services de santé et les disparités régionales dans l'état de santé ou les résultats des soins. Les premières ne sont pas nécessairement préjudiciables, dans la mesure où elles ne reflètent que des moyens différents de parvenir aux mêmes fins et où les secondes sont réduites au minimum. Toutefois, si des disparités régionales notables persistaient dans l'état de santé, il faudrait remettre en question la méthode de prestation de services ou les niveaux de consommation des services. Ceci dit, même si nous avons utilisé les RMR et les AR comme groupe de référence aux fins de comparaison avec les catégories rurales, nous n'estimons pas pour autant que les taux d'utilisation observés dans les régions urbaines devraient être la norme vers laquelle les régions rurales devraient tendre.

\section{Système de santé et santé rurale}

Quoique des différences existent entre les collectivités rurales et les collectivités urbaines, il ne faudrait pas en exagérer l'importance, pas plus qu'il ne faudrait en déduire que la situation dans les régions rurales est toujours mauvaise ou, du moins, est pire que celle qui prévaut dans les régions urbaines. Si, de manière générale, les résidents ruraux ont un accès moins facile à certains types de services de santé ou utilisent moins certains types de services, pour le reste il n'y a pas de différence notoire entre les régions rurales et urbaines. Parfois, les résidents ruraux utilisent même davantage certains types de services que ne le font les résidents urbains.

Le système de santé canadien, qui garantit l'universalité des soins hospitaliers et des soins médicaux fondamentaux, et la Loi canadienne sur la santé, qui compte l'« accessibilité » parmi ses cinq principes, visent à ce que les ressources financières ne soient pas une condition d'obtention de soins hospitaliers et de soins médicaux fondamentaux. L'universalité des soins exige que les praticiens et les services soient disponibles dans toutes les régions du pays. C'est pour cette raison que presque tous les gouvernements provinciaux et territoriaux ont créé des programmes spéciaux (p. ex. le Programme des services aux régions insuffisamment desservies en Ontario, le Fly-In Program 
de la J.A. Hildes Northern Medical Unit au Manitoba et le Travel Assistance Program en Colombie-Britannique) destinés à ceux qui doivent parcourir de grandes distances pour se faire soigner. Ces programmes (et d'autres) aident les résidents ruraux, particulièrement des régions les plus éloignées, à obtenir un accès plus facile aux services de santé et contribuent ainsi à réduire l'iniquité. Tous ces efforts témoignent de la vigueur du système de santé canadien.

Néanmoins, il faut noter que la présente étude s'est surtout concentrée sur les services de santé assurés, en particulier ceux fournis par les médecins et les hôpitaux. Les services non couverts par le système de santé, par exemple la réadaptation fonctionnelle, les soins à domicile, les soins dentaires et la santé mentale communautaire, n’ont pas été examinés en détail, principalement en raison de la difficulté d'obtenir des données. Par conséquent, on ne sait pas encore très bien si, à l'échelle nationale, les résidents ruraux ont un meilleur ou un moins bon accès à ces services que les résidents urbains, et s'ils utilisent ces services dans la même mesure que les résidents urbains. Il faudra poursuivre les recherches lorsque les données seront disponibles.

On ignore aussi si les résidents urbains et ruraux ont la même facilité (ou difficulté) à se prévaloir des services de santé. Si, en théorie, tous les Canadiens ont accès aux soins hospitaliers et médicaux fondamentaux, certains pourraient n'y avoir accès qu'au prix de beaucoup de désagréments et de difficultés. Par exemple, les résidents des collectivités éloignées ont le même droit aux soins de spécialistes que ceux qui vivent dans les grands centres urbains, mais ils ont souvent une grande distance à parcourir pour bénéficier d'une consultation. Cela pourrait les conduire à des absences au travail, des pertes de revenu, des frais de déplacement importants et une plus grande détresse émotionnelle. En d'autres mots, l'accessibilité est une chose, mais son prix (financier et psychologique) en est une autre. En raison du manque de données, nous n'avons pas pu traiter ces questions dans cette étude.

\section{Causes des variations}

régionales d'utilisation

Conformément au modèle d'Andersen², les variations régionales dans l'utilisation des services pourraient tenir, entre autres, à des besoins médicaux différents et à des variations dans la disponibilité des ressources en soins de santé. Par exemple, nous avons observé des risques relatifs de consultations chez le médecin et d'hospitalisation pour cause de traumatismes ou d'intoxications plus grands dans les régions rurales, tant à l'échelle nationale que dans les trois provinces examinées. Ces risques plus élevés traduisent peut-être la probabilité accrue de subir des accidents ou des blessures en raison des métiers associés à l'agriculture, à la pêche, à l'exploitation forestière ou à l'extraction minière. De même, le risque relatif beaucoup plus élevé de consultations ambulatoires liées au diabète dans les ZIM nulles observé dans les trois provinces pourrait être attribuable à la prévalence plus grande du diabète chez les Autochtones, lesquels composent une grande proportion de la population des régions les plus éloignées.

Les différences observées dans la disponibilité des ressources et dans les modèles de prestation des services influent beaucoup sur l'utilisation des services. Par exemple, l'offre limitée en soins communautaires, la charge de morbidité plus lourde et d'autres facteurs comme l'éloignement des services pourraient accroître la dépendance à l'égard des hôpitaux chez les Canadiens ruraux.

\section{Rôle du « lieu » en santé}

Est-ce que le lieu de résidence influe sur l'accès aux services de santé et leur utilisation? D’après les résultats de la présente étude et ceux de la précédente, intitulée Comment se portent les Canadiens vivant en milieu rural $?^{1}$, il faut répondre à cette question par l'affirmative : «Oui, le lieu de résidence importe, dans certains cas ». Une série d'analyses de régression multivariées a montré, lorsqu'on tient compte de divers facteurs sociodémographiques, de certaines maladies et des comportements en matière de santé, que le lieu de résidence, qu'il soit urbain ou rural, a un effet indépendant sur un certain nombre d'aspects de l'utilisation des services de santé.

Mais l'importance du lieu de résidence dépend également des variables choisies. C'est un facteur important dans certains cas, négligeable dans d'autres. Par exemple, les données tirées de l'Enquête sur l'accès aux services de santé révèlent que le lieu de résidence a un effet indépendant sur le fait de ne pas avoir de médecin de famille seulement dans les ZIM nulles, les autres variables étant contrôlées (figure 2). Par ailleurs, le lieu de résidence a un effet indépendant sur la probabilité d'être hospitalisé dans toutes les ZIM sauf dans les ZIM fortes (tableau 9).

Mais quelles caractéristiques du lieu de résidence font qu'il influe sur les comportements et les résultats en matière de santé? C'est une question tout aussi importante, et à laquelle il est sans doute plus difficile de répondre. Comme nous l'avons déjà vu, le « lieu » a de multiples significations : l'environnement physique, la population, les conditions socioéconomiques, les activités professionnelles, la culture, les coutumes, la structure communautaire, les relations sociales, etc. Par conséquent, lorsque nous parlons du rôle du lieu de résidence en santé, nous parlons en fait de la façon dont la santé est influencée par des facteurs interactifs qui se retrouvent réunis dans des endroits géographiques donnés. Puisque la présente étude, ainsi que d'autres, a établi qu'il importe de tenir compte du lieu pour comprendre la santé, il nous appartient d'aller au-delà de la simple ruralité et d'examiner comment ces facteurs interactifs influent sur la santé - et sont influencés par elle - dans le Canada rural.

De plus, la « ruralité » n'est qu'un aspect du « lieu » en lien avec la santé. On s'intéresse de plus en plus à d'autres aspects du lieu de résidence et à leurs rapports avec la santé, comme l'illustre le nombre croissant d'ouvrages sur la santé urbaine en général, la santé en centre-ville, la santé circumpolaire et la santé frontalière (p. ex. dans les régions longeant la frontière 
américano-mexicaine). Nous comprendrions mieux la santé rurale si nous en savions davantage sur la santé dans les quartiers, dans les centres-villes, dans les banlieues, aux frontières, dans les régions isolées, etc.

\section{Que faire maintenant?}

Si cette étude, tout comme la première ${ }^{1}$, a traité de très nombreux points, on ignore encore beaucoup de choses à propos de la santé des Canadiens ruraux et de la façon dont ils utilisent les services de santé. Voici quelques points qui pourraient guider les travaux futurs, pour une meilleure compréhension de la santé rurale et de l'utilisation des services de santé.

- La « ruralité » n’est pas un concept monolithique. Sur le plan de l'accès aux services de santé et de leur utilisation, les ZIM fortes ressemblent davantage aux villes, les ZIM nulles étant à l'autre extrémité et les ZIM modérées et faibles quelque part entre les deux. Doit-on accorder une attention et un soutien tout particuliers aux habitants des ZIM nulles et faibles, étant donné que ce sont eux qui pourraient avoir le plus besoin d'aide et disposer de moins de choix? En ce cas, quels types de soutien devraient être accordés et quelles autres données devraient être recueillies pour déterminer avec précision ces besoins et ce soutien?

- Les différences dans la prestation et l'utilisation des services de santé entre les régions rurales et urbaines ne sont pas nécessairement préjudiciables et pourraient même témoigner de conditions et de besoins différents. Ce qui est efficace dans les grandes villes ne l'est pas nécessairement dans les collectivités éloignées. Ce qui est indispensable dans les régions urbaines ne l'est peut-être pas dans les régions rurales. Il faut pousser davantage les recherches pour découvrir si les modes de prestation des services utilisés dans les régions rurales sont adaptés à la situation sociale et à l'état de santé des habitants. Il serait aussi intéressant de déterminer si certaines méthodes adoptées par les collectivités rurales pourraient servir de modèles aux régions urbaines canadiennes.

- Des variations régionales dans la prestation des services de santé sont acceptables, mais pas des disparités importantes et persistantes dans l'état de santé de la population. De nombreuses études sur la santé rurale, y compris Comment se portent les Canadiens vivant en milieu rural? ${ }^{1}$, ont fait état du moins bon état de santé des Canadiens ruraux. Des variations régionales importantes et persistantes dans l'état de santé de la population devraient être interprétées comme un signe de problème ou de dysfonctionnement dans les stratégies de prestation des soins de santé. Cela devrait nous inciter à suivre sur des périodes plus longues l'évolution des conditions de santé en milieu rural et à faire des études longitudinales de l'état de santé de la population rurale.

- La présente étude a surtout porté sur les services de santé assurés, étant donné que les données sur ces services sont plus facilement disponibles. Les différences sont peut-être encore plus grandes entre les Canadiens ruraux et urbains en ce qui a trait à l'accès aux services non assurés et à l'utilisation des services non assurés (tels que les soins dentaires, les soins de la vue et la réadaptation fonctionnelle), étant donné que ces services ne sont généralement pas remboursés et que certains sont très peu accessibles dans les collectivités rurales. Les futures recherches sur la santé rurale ne devraient pas se borner aux services couverts par le régime national d'assurance-santé. Il faudrait mettre rapidement sur pied un meilleur système de collecte des données portant sur les services autres que ceux des médecins et des hôpitaux.

- À cause du manque de données, la présente étude ne s'est pas focalisée non plus sur la promotion de la santé, la prévention des maladies, l'intervention précoce ou les mesures de prévention des accidents, des blessures et des suicides. Du point de vue de la santé de la population, ces services préventifs sont aussi importants que les services curatifs. Si les services curatifs et correctifs sont essentiels, il importe néanmoins de s'attaquer aux déterminants négatifs ayant un effet néfaste sur la santé de la population. Nous en savons encore moins sur ces soins non curatifs en région rurale, d'où la nécessité d'en faire une priorité de recherche.

- Les variations dans l'accès et dans l'utilisation des services de santé dépendent vraisemblablement de nombreux facteurs, pas simplement de l'absence de services ou de ressources à proximité. Par exemple, le manque de moyens de transport pourrait dissuader certains résidents ruraux de se prévaloir de soins qui ne sont offerts que dans des centres urbains éloignés. Cela signifie que les solutions ne relèvent pas exclusivement du domaine de la santé, et que l'amélioration des transports pourrait par exemple être tout aussi importante. C'est pourquoi les recherches en santé rurale devraient se pencher sur des facteurs généralement non reliés à la santé, mais pouvant néamoins éclairer les modalités d'accès des Canadiens ruraux aux services de santé et à leur utilisation.

- La nature des données et des méthodes utilisées dans cette étude ne permet pas de démêler les relations complexes qui existent entre les ressources en soins de santé, les modes de prestation des services de santé et la charge de morbidité, ni de déterminer leur incidence relative sur l'utilisation des services. De plus, il est impossible d'estimer ici dans quelle mesure l'utilisation des services de santé est un déterminant de l'état de santé chez les Canadiens ruraux. Cependant, si on peut dire sans risque d'erreur que les services de santé sont importants pour l'amélioration de l'état de santé des Canadiens en milieu rural, notamment en raison de la charge de morbidité généralement plus lourde, il est plus difficile de déterminer l'ampleur de cette importance. Ces éléments comptent dans la recherche et la planification en santé rurale et il faut les examiner de plus près. 
- Il est probablement impossible d'obtenir une égalité parfaite dans la répartition des ressources en santé et l'accès aux soins, mais nous ne savons pas quel degré d'inégalité est acceptable. Cette question relève de la politique tout autant que de la philosophie ou de l'éthique. La société pourrait avoir à trouver un équilibre entre la facilité d'accès, d'une part, et la viabilité économique, l'aspect pratique et la qualité des soins, d'autre part. Les Canadiens ruraux devraient être écoutés à ce propos. Les personnes qui s'intéressent à l'éthique en santé devraient aussi participer à cet important débat et lancer des recherches sur les enjeux éthiques en santé rurale.

- Comme nous l'avons mentionné, la " ruralité » n'est qu'un caractère du « lieu ». On s'intéresse de plus en plus à la façon dont différents aspects du « lieu » influent sur l'état de santé et sur les comportements en matière de santé. Au bout du compte, on aimerait savoir s'il existe des principes sous-jacents ou des cadres théoriques communs susceptibles de guider la recherche en santé rurale, en santé dans les centres-villes, en santé dans les régions éloignées, en santé circumpolaire, etc., et susceptibles également d'aider à mieux comprendre les relations entre le "lieu » et la santé. À cette fin, les chercheurs en santé rurale doivent travailler de concert avec les chercheurs en santé s'intéressant à d'autres aspects du "lieu ", pour créer une synergie dans la recherche et progresser sur le plan théorique.

Pour accroître l'équité en santé pour tous les Canadiens quel que soit leur lieu de résidence, nous devons mieux comprendre les variations dans l'état de santé et dans l'utilisation des services de santé. Pour cela, nous nous sommes ici attardés aux variations dans l'utilisation des services de santé, tandis que le premier rapport Comment se portent les Canadiens vivant en milieu rural? $?^{1}$ s'est surtout penché sur les variations dans l'état de santé. Nous avons examiné en détail la façon dont les Canadiens ruraux accèdent à un large éventail de services de santé et utilisent ces services. Nous nous sommes servis de plusieurs sources de données, de différents types (données d'enquête ou administratives), et avons fait des analyses à l'échelle nationale et provinciale. Nous avons examiné l'utilisation des services de santé à grande échelle et en rapport avec un certain nombre de catégories de maladies. Allant au-delà de la simple dichotomie rural-urbain, nous avons scindé le milieu rural en catégories plus fines pour mieux comprendre les variations intrarurales dans la consommation des soins de santé. Nous espérons qu'en offrant un éclairage neuf sur les modalités d'utilisation des services en soins de santé et en répondant aux questions laissées précédemment en suspens, la présente étude contribuera à l'amélioration des soins de santé fournis aux Canadiens ruraux et à une meilleure compréhension du rôle du lieu.

\section{Remerciements}

L'étude a été financée par l'Initiative sur la santé de la population canadienne (ISPC) de l'Institut canadien d'information sur la santé (ICIS), en collaboration avec l'Agence de la santé publique du Canada (ASPC), le Centre de recherche en santé dans les milieux ruraux et du Nord de l'Université Laurentienne (CReSRN) et autres chercheurs. Les points de vue exprimés ne sont pas nécessairement ceux de l'ISPC, de l'ICIS, de l'ASPC ou du CReSRN.

L'étude a aussi été appuyée par l'Institut de recherche en services de santé (IRSS), qui est financé par une subvention annuelle du ministère de la Santé et des Soins de longue durée de l'Ontario (MSSLDO). Cependant, les opinions, résultats et conclusions qui s'en dégagent sont ceux des auteurs et sont indépendants des sources de financement : ils ne reflètent pas forcément la position de l'IRSS ou celle du MSSLDO.

\section{Références}

1. Institut canadien d'information sur la santé. Comment se portent les Canadiens vivant en milieu rural? Une évaluation de leur état de santé et des déterminants de la santé, Ottawa (Ontario), Institut canadien d'information sur la santé, 2006.
2. Andersen, R.M. " Revisiting the behavioral model and access to medical care: does it matter? ", J Health Soc Behav. 1995;36(1):1-10.

3. Pong, R.W., J. R. Pitblado. Répartition géographique des médecins au Canada: au-delà du nombre et du lieu, Ottawa (Ontario), Institut canadien d'information sur la santé, 2005.

4. Chan, B.T., P.C. Austin. « Patient, physician, and community factors affecting referrals to specialists in Ontario, Canada: a populationbased, multi-level modelling approach ", Medical Care, 2003;41(4):500-11.

5. Mathers, C.D., D.J. Schofield. « The health consequences of unemployment: the evidence ", The Medical Journal of Australia, 1998;168(4):178-82.

6. Malenfant, E.C., A. Milan, M. Charron et A. Bélanger. Changements démographiques au Canada de 1971 à 2001 selon un gradient urbain-rural, Ottawa (Ontario), Statistique Canada, 2007, nº 91F0015MIF au catalogue $\mathrm{n}^{\circ} 008$.

7. Molinari C., M. Ahern et M. Hendryx. «The relationship of community quality to the health of women and men ", Social Science \& Medicine, 1998;47(8):1113-20.

8. Andersen M.R., N. Urban et R. Etzioni. " Community attitudes and mammography use: does it really matter what other people think? ", Women \& Health, 1999;29(3):83-95.

9. Humphreys J.S., S. Mathews-Cowey et H.C. Weinand. "Factors in accessibility of general practice in rural Australia ", Medical Journal of Australia, 1997;166(11):557-80.

10. Dixon J., N. Welch. « Researching the ruralmetropolitan health differential using the 'social determinants of health' ", Australian Journal of Rural Health, 2000;8(5):254-60.

11. Arcury T.A., J.S. Preisser, W.M. Gesler et J.M. Powers. "Access to transportation and health care utilization in a rural region », The Journal of Rural Health, 2005; 21(1):31-8. 
12. Gupta S., L.L. Roos, R. Walld, D. Traverse et M. Dahl, « Delivering equitable care: comparing preventive services in Manitoba ", American Journal of Public Health, 2003; 93(12):2086-92.

13. Martens P.J., R. Fransoo, E. Burland, L. Jebamani, C. Burchill, C. Black, N. Dik, L. MacWilliam, S. Derksen, R. Walld, C. Steinbach, M. Dahl, et B. Bogdanovic. The Need to Know Team. The Manitoba RHA indicators atlas: population-based comparisons of health and health use, Winnipeg (Manitoba), Manitoba Centre for Health Policy, 2003.

14. Stewart F.J., D.A. Rosenthal. Rural and urban female secondary school students' attitudes towards and use of primary care services, Australian Journal of Rural Health, 1997; 5(3):126-31.

15. Centre de prévention et de contrôle des maladies chroniques. Approche intégrée des maladies chroniques [Internet], Ottawa (Ontario), Agence de santé publique du Canada [cité le 15 mars 2006; dernière mise à jour le 15 mai 2009]. Accessible à l'adresse http://web.archive.org/web/ 20041205003337/www.phac-aspc.gc.ca/ ccdpc-cpcmc/topics/integrated_f.html.

16. Martinez J., R. Pampalon, D. Hamel et G. Raymond. Vivre dans une collectivité rurale plutôt qu'en ville fait-il vraiment une différence en matière de santé et de bien-être? Québec (Québec), Institut national de santé publique du Québec, 2004, rapport nº 269.

17. Goel V., J.I. Williams, G.M. Anderson, P. Blackstien-Hirsch, C. Fooks et C.D. Naylor. (éditeurs). Patterns of health care in Ontario: the ICES practice atlas. $2^{e}$ éd., Ottawa (Ontario), publié pour l'Institut de recherche en services de santé de l'Ontario par l'Association médicale canadienne, 1996.

18. Eberhardt M.S., D.D. Ingram et D.M. Makuc. Urban and rural health chartbook: Health, États-Unis, 2001, Hyattsville (MD), National Center for Health Statistics, 2001.
19. Institut canadien d'information sur la santé. Comprendre les temps d'attente dans les services d'urgence: qui utilise les services d'urgence et quels sont les temps d'attente?, Ottawa (Ontario), Institut canadien d'information sur la santé, 2005, français et anglais.

20. du Plessis V., R. Beshiri, R. D. Bollman et $\mathrm{H}$. Clemenson. Définitions de "rural " [Internet], Ottawa (Ontario), Statistique Canada, Division de l'agriculture, 2002 [cité 15 mars 2007], Série de documents de travail sur l'agriculture et le milieu rural, $\mathrm{n}^{\mathrm{o}}$ 61), accessible à l'adresse http:// www.statcan.gc.ca/bsolc/olc-cel/ olc-cel?catno $=$ 21-601-M2002061\&lang $=$ fra .

21. McNiven C., H. Puderer et D. Janes. Zones d'influence des régions métropolitaines de recensement et des agglomérations de recensement (ZIM) : une description de la méthodologie, Ottawa (Ontario), Division de la géographie, Statistique Canada, 2000 (Série de documents de travail de la géographie, no 2000-2).

22. Rambeau S., K. Todd. Zones d'influence des régions métropolitaines de recensement et des agglomérations de recensement (ZIM) accompagnées de données de recensement [Internet], Ottawa (Ontario), Statistique Canada, 2000 [cité 15 avril 2009], téléchargeable [PDF] à l'adresse http://www. statcan.gc.ca/pub/92f0138m/2000001/ 4274061-fra.pdt.

23. Breslow N.E., N.E. Day. Statistical methods in cancer research. Vol. 2, The design and analysis of cohort studies, Lyon, Centre international de recherche sur le cancer, 1987, Scientific Publication $n^{\circ} 82$.

24. Commission sur l'avenir des soins de santé au Canada. Guidé par nos valeurs : l'avenir des soins de santé au Canada, Ottawa (Ontario), Gouvernement du Canada, 2002, $\mathrm{n}^{\circ}$ CP32-85/2002F-IN au catalogue. 


\section{Annexe 1 Glossaire}

AR Agglomération de recensement

BDMH Base de données sur la morbidité hospitalière

CCA Classification canadienne des actes diagnostiques, thérapeutiques et chirurgicaux

CCI Classification canadienne des interventions en santé

CIM-9 Classification statistique internationale des maladies, traumatismes et causes de décès, neuvième révision

CIM-10-CA Classification statistique internationale des maladies et des problèmes de santé connexes, dixième révision Améliorations canadiennes à la CIM-10

EASS Enquête sur l'accès aux services de santé

ESCC Enquête sur la santé dans les collectivités canadiennes

ICIS Institut canadien d'information sur la santé

MF $\quad$ Médecin de famille

RMR Région métropolitaine de recensement

RRPV Région rurale et petite ville

SDR Subdivision de recensement

ZIM Zone d’influence métropolitaine 


\section{Annexe 2 Profils provinciaux par maladie : tableaux détaillés}

\section{TABLEAU A1}

Risques relatifs de consultation chez le médecin (normalisée selon l'âge) pour maladies de la circulation, selon le lieu de résidence et le sexe, Ontario et Nouvelle-Écosse, 2001-2002, et Colombie-Britannique, 2000-2001

\begin{tabular}{|c|c|c|c|c|c|c|c|c|}
\hline & \multicolumn{4}{|c|}{ Hommes } & \multicolumn{4}{|c|}{ Femmes } \\
\hline & ZIM forte & $\begin{array}{c}\text { ZIM } \\
\text { modérée }\end{array}$ & ZIM faible & ZIM nulle & ZIM forte & $\begin{array}{c}\text { ZIM } \\
\text { modérée }\end{array}$ & ZIM faible & ZIM nulle \\
\hline Ontario & $\begin{array}{l}0,92 \\
(0,92-0,92)^{*}\end{array}$ & $\begin{array}{l}0,92 \\
(0,92-0,92)^{*}\end{array}$ & $\begin{array}{l}0,84 \\
(0,84-0,85)^{*}\end{array}$ & $\begin{array}{l}0,99 \\
(0,97-1,00)\end{array}$ & $\begin{array}{l}1,01 \\
(1,01-1,01)^{*}\end{array}$ & $\begin{array}{l}0,97 \\
(0,96-0,97)^{*}\end{array}$ & $\begin{array}{l}0,93 \\
(0,92-0,93)^{*}\end{array}$ & $\begin{array}{l}0,83 \\
(0,81-0,85)^{*}\end{array}$ \\
\hline Nouvelle-Écosse & $\begin{array}{l}1,19 \\
(1,17-1,21)^{*}\end{array}$ & $\begin{array}{l}0,97 \\
(0,96-0,98)^{*}\end{array}$ & $\begin{array}{l}0,99 \\
(0,98-1,00)^{*}\end{array}$ & $\begin{array}{l}0,89 \\
(0,85-0,93)^{*}\end{array}$ & $\begin{array}{l}1,33 \\
(1,31-1,35)^{*}\end{array}$ & $\begin{array}{l}1,04 \\
(1,03-1,05)^{*}\end{array}$ & $\begin{array}{l}1,06 \\
(1,05-1,07)^{*}\end{array}$ & $\begin{array}{l}0,87 \\
(0,83-0,91)^{*}\end{array}$ \\
\hline \multicolumn{9}{|c|}{ Maladie ischémique cardiaque $(410-414)^{\mathrm{a}}$} \\
\hline Colombie-Britannique & $\begin{array}{l}0,68 \\
(0,65-0,71)^{*}\end{array}$ & $\begin{array}{l}0,83 \\
(0,81-0,85)^{*}\end{array}$ & $\begin{array}{l}0,60 \\
(0,59-0,62)^{*}\end{array}$ & $\begin{array}{l}0,88 \\
(0,82-0,93)^{*}\end{array}$ & $\begin{array}{l}0,88 \\
(0,83-0,92)^{*}\end{array}$ & $\begin{array}{l}0,84 \\
(0,82-0,87)^{*}\end{array}$ & $\begin{array}{l}0,67 \\
(0,65-0,70)^{*}\end{array}$ & $\begin{array}{l}0,98 \\
(0,91-1,06)\end{array}$ \\
\hline Nouvelle-Écosse & $\begin{array}{l}1,15 \\
(1,09-1,20)^{*}\end{array}$ & $\begin{array}{l}1,02 \\
(0,99-1,04)\end{array}$ & $\begin{array}{l}1,01 \\
(0,99-1,03)\end{array}$ & $\begin{array}{l}1,06 \\
(0,94-1,19)\end{array}$ & $\begin{array}{l}1,46 \\
(1,36-1,56)^{*}\end{array}$ & $\begin{array}{l}1,13 \\
(1,09-1,18)^{*}\end{array}$ & $\begin{array}{l}1,02 \\
(0,99-1,05)\end{array}$ & $\begin{array}{l}0,78 \\
(0,64-0,95)^{*}\end{array}$ \\
\hline Nouvelle-Écosse & $\begin{array}{l}0,88 \\
(0,57-1,38)\end{array}$ & $\begin{array}{l}1,20 \\
(0,98-1,46)\end{array}$ & $\begin{array}{l}1,07 \\
(0,92-1,25)\end{array}$ & $\begin{array}{l}0,51 \\
(0,14-1,94)\end{array}$ & $\begin{array}{l}1,78 \\
(1,17-2,70)^{*}\end{array}$ & $\begin{array}{l}1,68 \\
(1,35-2,09)^{*}\end{array}$ & $\begin{array}{l}1,43 \\
(1,20-1,71)^{*}\end{array}$ & $\begin{array}{l}1,17 \\
(0,40-3,39)\end{array}$ \\
\hline
\end{tabular}

Sources des données : Base de données sur la facturation des médecins de la Nouvelle-Écosse, 2001-2002; Base de données sur la facturation des médecins de l'Ontario, 2001-2002; Régime des services médicaux de la Colombie-Britannique, 2000-2001.

Abréviations : AR, agglomération de recensement; RMR, région métropolitaine de recensement; CIM-9, Classification statistique internationale des maladies, traumatismes et causes de décès, neuvième édition; ZIM, zone d'influence métropolitaine.

a Codes de diagnostic de la CIM-9.

* Statistiquement significatif à $p<0,05$; le groupe de référence est la RMR-AR (risque $=1,00$ ).

TABLEAU A2

Risques relatifs de congé d'hôpital (normalisé selon l'âge) pour maladies de la circulation, selon le lieu de résidence et le sexe, Ontario, Nouvelle-Écosse et Colombie-Britannique, 2001-2002

\begin{tabular}{llcccc}
\hline Province & Sexe & ZIM forte & ZIM modérée & ZIM faible & ZIM nulle \\
\hline \multirow{2}{*}{ Ontario } & Hommes & $1,15(1,10-1,20)^{*}$ & $1,28(1,25-1,32)^{*}$ & $1,58(1,54-1,61)^{*}$ & $2,10(1,95-2,25)^{*}$ \\
& Femmes & $1,27(1,23-1,31)^{*}$ & $1,48(1,43-1,53)^{*}$ & $1,91(1,83-2,00)^{*}$ & $2,10(1,90-2,31)^{*}$ \\
\hline \multirow{2}{*}{ Nouvelle-Écosse } & Hommes & $1,88(1,71-2,06)^{*}$ & $1,33(1,24-1,42)^{*}$ & $1,19(1,13-1,26)^{*}$ & $3,38(2,83-4,02)^{*}$ \\
& Femmes & $2,38(2,12-2,68)^{*}$ & $1,88(1,73-2,03)^{*}$ & $1,46(1,36-1,56)^{*}$ & $5,79(4,86-6,90)^{*}$ \\
\hline \multirow{2}{*}{ Colombie-Britannique } & Hommes & $1,01(0,92-1,10)$ & $1,02(0,96-1,07)$ & $1,29(1,24-1,35)^{*}$ & $1,82(1,64-2,02)^{*}$ \\
& Femmes & $1,39(1,25-1,54)^{*}$ & $1,29(1,21-1,38)^{*}$ & $1,70(1,61-1,79)^{*}$ & $2,37(2,08-2,69)^{*}$ \\
\hline
\end{tabular}

Source des données : Base de données sur la morbidité hospitalière, 2001-2002, Institut canadien d'information sur la santé.

Abréviations : AR, agglomération de recensement; RMR, région métropolitaine de recensement; ZIM, zone d'influence métropolitaine.

* Statistiquement significatif à $p<0,05$; le groupe de référence est la RMR-AR (risque $=1,00$ ). 
TABLEAU A3

Risques relatifs de consultation chez le médecin (normalisée selon l'âge) pour cancers (néoplasmes), selon le lieu de résidence et le sexe, Ontario et Nouvelle-Écosse, 2001-2002, et Colombie-Britannique, 2000-2001

\begin{tabular}{|c|c|c|c|c|c|c|c|c|}
\hline & \multicolumn{4}{|c|}{ Hommes } & \multicolumn{4}{|c|}{ Femmes } \\
\hline & ZIM forte & $\begin{array}{c}\text { ZIM } \\
\text { modérée }\end{array}$ & ZIM faible & ZIM nulle & ZIM forte & $\begin{array}{c}\text { ZIM } \\
\text { modérée }\end{array}$ & ZIM faible & ZIM nulle \\
\hline \multicolumn{9}{|l|}{ Néoplasmes (140-239)a } \\
\hline Ontario & $\begin{array}{l}0,95 \\
(0,94-0,96)^{*}\end{array}$ & $\begin{array}{l}0,99 \\
(0,98-1,00)^{*}\end{array}$ & $\begin{array}{l}0,91 \\
(0,90-0,92)^{*}\end{array}$ & $\begin{array}{l}0,98 \\
(0,94-1,02)\end{array}$ & $\begin{array}{l}0,92 \\
(0,92-0,93)^{*}\end{array}$ & $\begin{array}{l}0,85 \\
(0,84-0,86)^{*}\end{array}$ & $\begin{array}{l}0,90 \\
(0,88-0,91)^{*}\end{array}$ & $\begin{array}{l}1,12 \\
(1,08-1,16)^{*}\end{array}$ \\
\hline Nouvelle-Écosse & $\begin{array}{l}1,09 \\
(1,05-1,14)^{*}\end{array}$ & $\begin{array}{l}0,94 \\
(0,92-0,97)^{*}\end{array}$ & $\begin{array}{l}0,83 \\
(0,82-0,85)^{*}\end{array}$ & $\begin{array}{l}1,01 \\
(0,91-1,11)\end{array}$ & $\begin{array}{l}0,97 \\
(0,93-1,02)\end{array}$ & $\begin{array}{l}0,97 \\
(0,95-1,00)^{*}\end{array}$ & $\begin{array}{l}0,79 \\
(0,78-0,81)^{*}\end{array}$ & $\begin{array}{l}0,76 \\
(0,68-0,85)^{*}\end{array}$ \\
\hline \multicolumn{9}{|c|}{ Néoplasme du sein (174) } \\
\hline Colombie-Britannique & s.o. & S.o. & S.o. & S.o. & $\begin{array}{l}0,87 \\
(0,79-0,95)^{*}\end{array}$ & $\begin{array}{l}1,24 \\
(1,19-1,31)^{*}\end{array}$ & $\begin{array}{l}1,29 \\
(1,24-1,35)^{*}\end{array}$ & $\begin{array}{l}2,23 \\
(2,03-2,44)^{*}\end{array}$ \\
\hline Nouvelle-Écosse & S.o. & S.o. & S.o. & S.o. & $\begin{array}{l}0,81 \\
(0,72-0,92)^{*}\end{array}$ & $\begin{array}{l}0,87 \\
(0,82-0,92)^{*}\end{array}$ & $\begin{array}{l}0,82 \\
(0,78-0,85)^{*}\end{array}$ & $\begin{array}{l}0,55 \\
(0,40-0,75)^{*}\end{array}$ \\
\hline \multicolumn{9}{|c|}{ Néoplasme du poumon (162)a } \\
\hline Colombie-Britannique & $\begin{array}{l}0,91 \\
(0,78-1,07)\end{array}$ & $\begin{array}{l}1,28 \\
(1,18-1,39)^{*}\end{array}$ & $\begin{array}{l}1,23 \\
(1,14-1,33)^{*}\end{array}$ & $\begin{array}{l}1,67 \\
(1,39-2,01)^{*}\end{array}$ & $\begin{array}{l}0,89 \\
(0,74-1,07)\end{array}$ & $\begin{array}{l}1,45 \\
(1,33-1,58)^{*}\end{array}$ & $\begin{array}{l}1,01 \\
(0,92-1,11)\end{array}$ & $\begin{array}{l}2,81 \\
(2,39-3,31)^{*}\end{array}$ \\
\hline Nouvelle-Écosse & $\begin{array}{l}1,16 \\
(0,97-1,40)\end{array}$ & $\begin{array}{l}0,97 \\
(0,88-1,08)\end{array}$ & $\begin{array}{l}1,23 \\
(1,15-1,32)^{*}\end{array}$ & $\begin{array}{l}2,34 \\
(1,75-3,14)^{*}\end{array}$ & $\begin{array}{l}0,84 \\
(0,65-1,09)\end{array}$ & $\begin{array}{l}0,95 \\
(0,85-1,08)\end{array}$ & $\begin{array}{l}0,95 \\
(0,87-1,04)\end{array}$ & $\begin{array}{l}0,36 \\
(0,16-0,82)^{*}\end{array}$ \\
\hline
\end{tabular}

Sources des données : Base de données sur la facturation des médecins de la Nouvelle-Écosse, 2001-2002; Base de données sur la facturation des médecins de l’Ontario, 2001-2002; Régime des services médicaux de la Colombie Britannique, 2000-2001

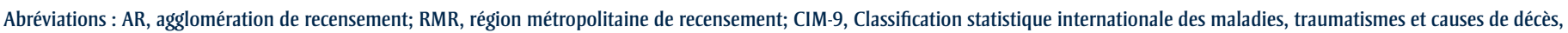
neuvième édition; ZIM, zone d'influence métropolitaine.

a Codes de diagnostic de la CIM-9.

* Statistiquement significatif à $p<0,05$; le groupe de référence est la RMR-AR (risque $=1,00$ ).

\section{TABLEAU A4}

Risques relatifs de congé d'hôpital (normalisé selon l'âge) pour cancers (néoplasmes), selon le lieu de résidence et le sexe, Ontario, Nouvelle-Écosse et Colombie-Britannique, 2001-2002

\begin{tabular}{|c|c|c|c|c|c|}
\hline Province & Sexe & ZIM forte & ZIM modérée & ZIM faible & ZIM nulle \\
\hline \multirow{2}{*}{ Ontario } & Hommes & $1,00(0,93-1,07)$ & $1,15(1,09-1,20)^{*}$ & $1,39(1,35-1,45)^{*}$ & $1,62(1,43-1,85)^{*}$ \\
\hline & Femmes & $1,06(1,02-1,10)^{*}$ & $1,19(1,14-1,24)^{*}$ & $1,17(1,10-1,24)^{*}$ & $1,34(1,16-1,54)^{*}$ \\
\hline \multirow{2}{*}{ Nouvelle-Écosse } & Hommes & $1,72(1,47-2,02)^{*}$ & $1,30(1,16-1,45)^{*}$ & $1,12(1,03-1,22)^{*}$ & $4,49(3,51-5,73)^{*}$ \\
\hline & Femmes & $1,69(1,44-1,98)^{*}$ & $1,44(1,30-1,59)^{*}$ & $1,24(1,15-1,35)^{*}$ & $2,85(2,14-3,80)^{*}$ \\
\hline \multirow{2}{*}{ Colombie-Britannique } & Hommes & $0,91(0,78-1,06)$ & $1,01(0,93-1,11)$ & $1,31(1,21-1,41)^{*}$ & $2,40(2,06-2,79)^{*}$ \\
\hline & Femmes & $0,87(0,75-1,00)$ & $0,88(0,81-0,96)^{*}$ & $1,11(1,04-1,20)^{*}$ & $1,53(1,28-1,83)^{*}$ \\
\hline
\end{tabular}

Source des données : Base de données sur la morbidité hospitalière, 2001-2002, Institut canadien d'information sur la santé.

Abréviations : AR, agglomération de recensement; RMR, région métropolitaine de recensement; ZIM, zone d'influence métropolitaine.

* Statistiquement significatif à $p<0,05$; le groupe de référence est la RMR-AR (risque $=1,00$ ). 
TABLEAU A5

Risques relatifs de consultation chez le médecin (normalisée selon l'âge) pour maladies du système respiratoire, selon le lieu de résidence et le sexe, Ontario et Nouvelle-Écosse, 2001-2002, et Colombie-Britannique, 2000-2001

\begin{tabular}{|c|c|c|c|c|c|c|c|c|}
\hline & \multicolumn{4}{|c|}{ Hommes } & \multicolumn{4}{|c|}{ Femmes } \\
\hline & ZIM forte & $\begin{array}{c}\text { ZIM } \\
\text { modérée }\end{array}$ & ZIM faible & ZIM nulle & ZIM forte & $\begin{array}{c}\text { ZIM } \\
\text { modérée }\end{array}$ & ZIM faible & ZIM nulle \\
\hline \multicolumn{9}{|c|}{ Maladie du système respiratoire (460-519) ${ }^{a}$} \\
\hline Ontario & $\begin{array}{l}0,71 \\
(0,70-0,71)^{*}\end{array}$ & $\begin{array}{l}0,68 \\
(0,67-0,68)^{*}\end{array}$ & $\begin{array}{l}0,65 \\
(0,65-0,66)^{*}\end{array}$ & $\begin{array}{l}0,58 \\
(0,57-0,59)^{*}\end{array}$ & $\begin{array}{l}0,79 \\
(0,79-0,79)^{*}\end{array}$ & $\begin{array}{l}0,74 \\
(0,74-0,75)^{*}\end{array}$ & $\begin{array}{l}0,71 \\
(0,71-0,72)^{*}\end{array}$ & $\begin{array}{l}0,59 \\
(0,58-0,60)^{*}\end{array}$ \\
\hline Nouvelle-Écosse & $\begin{array}{l}1,15 \\
(1,13-1,17)^{*}\end{array}$ & $\begin{array}{l}1,01 \\
(1,01-1,02)^{*}\end{array}$ & $\begin{array}{l}0,94 \\
(0,94-0,95)^{*}\end{array}$ & $\begin{array}{l}0,96 \\
(0,92-1,00)^{*}\end{array}$ & $\begin{array}{l}1,17 \\
(1,16-1,18)^{*}\end{array}$ & $\begin{array}{l}1,05 \\
(1,04-1,05)^{*}\end{array}$ & $\begin{array}{l}0,96 \\
(0,95-0,96)^{*}\end{array}$ & $\begin{array}{l}0,97 \\
(0,94-1,00)^{*}\end{array}$ \\
\hline \multicolumn{9}{|l|}{ Asthme (493) $)^{a}$} \\
\hline Colombie-Britannique & $\begin{array}{l}0,63 \\
(0,60-0,66)^{*}\end{array}$ & $\begin{array}{l}0,82 \\
(0,80-0,84)^{*}\end{array}$ & $\begin{array}{l}0,64 \\
(0,62-0,65)^{*}\end{array}$ & $\begin{array}{l}0,75 \\
(0,70-0,81)^{*}\end{array}$ & $\begin{array}{l}0,70 \\
(0,67-0,73)^{*}\end{array}$ & $\begin{array}{l}0,94 \\
(0,92-0,96)^{*}\end{array}$ & $\begin{array}{l}0,69 \\
(0,67-0,71)^{*}\end{array}$ & $\begin{array}{l}0,97 \\
(0,91-1,03)\end{array}$ \\
\hline Ontario & $\begin{array}{l}0,70 \\
(0,69-0,71)^{*}\end{array}$ & $\begin{array}{l}0,74 \\
(0,73-0,75)^{*}\end{array}$ & $\begin{array}{l}0,79 \\
(0,78-0,81)^{*}\end{array}$ & $\begin{array}{l}0,69 \\
(0,34-0,74)^{*}\end{array}$ & $\begin{array}{l}0,82 \\
(0,81-0,83)^{*}\end{array}$ & $\begin{array}{l}0,84 \\
(0,83-0,85)^{*}\end{array}$ & $\begin{array}{l}0,88 \\
(0,86-0,90)^{*}\end{array}$ & $\begin{array}{l}0,56 \\
(0,51-0,60)^{*}\end{array}$ \\
\hline Nouvelle-Écosse & $\begin{array}{l}1,05 \\
(0,99-1,12)\end{array}$ & $\begin{array}{l}0,83 \\
(0,81-0,86)^{*}\end{array}$ & $\begin{array}{l}0,82 \\
(0,80-0,84)^{*}\end{array}$ & $\begin{array}{l}0,78 \\
(0,66-0,92)^{*}\end{array}$ & $\begin{array}{l}1,13 \\
(1,07-1,19)^{*}\end{array}$ & $\begin{array}{l}0,83 \\
(0,81-0,86)^{*}\end{array}$ & $\begin{array}{l}0,83 \\
(0,81-0,85)^{*}\end{array}$ & $\begin{array}{l}0,76 \\
(0,66-0,88)^{*}\end{array}$ \\
\hline \multicolumn{9}{|c|}{ Maladie pulmonaire chronique obstructive $(490-492,496)^{a}$} \\
\hline Colombie-Britannique & $\begin{array}{l}0,77 \\
(0,74-0,81)^{*}\end{array}$ & $\begin{array}{l}0,87 \\
(0,84-0,89)^{*}\end{array}$ & $\begin{array}{l}0,90 \\
(0,88-0,93)^{*}\end{array}$ & $\begin{array}{l}1,21 \\
(1,14-1,28)^{*}\end{array}$ & $\begin{array}{l}0,78 \\
(0,75-0,82)^{*}\end{array}$ & $\begin{array}{l}0,93 \\
(0,90-0,95)^{*}\end{array}$ & $\begin{array}{l}0,96 \\
(0,93-0,98)^{*}\end{array}$ & $\begin{array}{l}1,33 \\
(1,23-1,41)^{*}\end{array}$ \\
\hline Nouvelle-Écosse & $\begin{array}{l}1,58 \\
(1,50-1,66)^{*}\end{array}$ & $\begin{array}{l}1,34 \\
(1,30-1,37)^{*}\end{array}$ & $\begin{array}{l}1,01 \\
(0,98-1,03)\end{array}$ & $\begin{array}{l}1,22 \\
(1,08-1,39)^{*}\end{array}$ & $\begin{array}{l}1,19 \\
(1,12-1,26)^{*}\end{array}$ & $\begin{array}{l}1,34 \\
(1,31-1,38)^{*}\end{array}$ & $\begin{array}{l}1,03 \\
(1,01-1,06)^{*}\end{array}$ & $\begin{array}{l}1,35 \\
(1,21-1,50)^{*}\end{array}$ \\
\hline
\end{tabular}

Sources des données : Base de données sur la facturation des médecins de la Nouvelle-Écosse, 2001-2002; Base de données sur la facturation des médecins de l’Ontario, 2001-2002; Régime des services médicaux de la Colombie Britannique, 2000-2001.

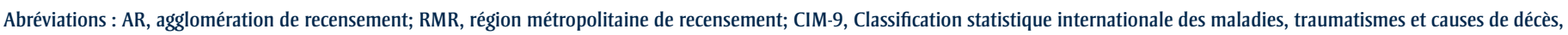
neuvième édition; ZIM, zone d'influence métropolitaine.

a Codes de diagnostic de la CIM-9.

Statistiquement significatif à $p<0,05$; le groupe de référence est la RMR-AR (risque $=1,00$ ).

\section{TABLEAU A6}

Risques relatifs de congé d'hôpital (normalisé selon l'âge) pour maladies du système respiratoire, selon le lieu de résidence et le sexe, Ontario, Nouvelle-Écosse et Colombie-Britannique, 2001-2002

\begin{tabular}{|c|c|c|c|c|c|}
\hline Province & Sexe & ZIM forte & ZIM modérée & ZIM faible & ZIM nulle \\
\hline \multirow{2}{*}{ Ontario } & Hommes & $1,20(1,13-1,28)^{*}$ & $1,45(1,39-1,50)^{*}$ & $1,72(1,67-1,77)^{*}$ & $2,32(2,10-2,56)^{*}$ \\
\hline & Femmes & $1,21(1,16-1,26)^{*}$ & $1,49(1,43-1,56)^{*}$ & $1,88(1,78-1,99)^{*}$ & $2,97(2,67-3,30)^{*}$ \\
\hline \multirow{2}{*}{ Nouvelle-Écosse } & Hommes & $1,27(1,09-1,48)^{*}$ & $1,18(1,08-1,30)^{*}$ & $1,31(1,22-1,40)^{*}$ & $3,00(2,35-3,82)^{*}$ \\
\hline & Femmes & $0,93(0,76-1,13)$ & $1,08(0,97-1,20)$ & $1,23(1,14-1,33)^{*}$ & $3,69(2,91-4,68)^{*}$ \\
\hline \multirow{2}{*}{ Colombie-Britannique } & Hommes & $0,88(0,78-1,00)^{*}$ & $0,96(0,89-1,03)$ & $1,71(1,62-1,81)^{*}$ & $1,47(1,26-1,72)^{*}$ \\
\hline & Femmes & $0,87(0,74-1,01)$ & $1,02(0,93-1,10)$ & $1,99(1,88-2,11)^{*}$ & $1,95(1,65-2,30)^{*}$ \\
\hline
\end{tabular}

Source des données : Base de données sur la morbidité hospitalière, 2001-2002, Institut canadien d'information sur la santé.

Abréviations : AR, agglomération de recensement; RMR, région métropolitaine de recensement; ZIM, zone d'influence métropolitaine.

* Statistiquement significatif à $p<0,05$; le groupe de référence est la RMR-AR (risque $=1,00$ ). 
TABLEAU A7

Risques relatifs de consultation chez le médecin (normalisée selon l'âge) pour maladies de l'appareil locomoteur et du tissu conjonctif, selon le lieu de résidence et le sexe, Ontario et Nouvelle-Écosse, 2001-2002, et Colombie-Britannique, 2000-2001

\begin{tabular}{|c|c|c|c|c|c|c|c|c|}
\hline & \multicolumn{4}{|c|}{ Hommes } & \multicolumn{4}{|c|}{ Femmes } \\
\hline & ZIM forte & $\begin{array}{c}\text { ZIM } \\
\text { modérée }\end{array}$ & ZIM faible & ZIM nulle & ZIM forte & $\begin{array}{c}\text { ZIM } \\
\text { modérée }\end{array}$ & ZIM faible & ZIM nulle \\
\hline Ontario & $\begin{array}{l}0,92 \\
(0,91-0,92)^{*}\end{array}$ & $\begin{array}{l}1,00 \\
(0,99-1,00)\end{array}$ & $\begin{array}{l}0,98 \\
(0,97-0,99)^{*}\end{array}$ & $\begin{array}{l}0,95 \\
(0,92-0,97)^{*}\end{array}$ & $\begin{array}{l}0,91 \\
(0,90-0,91)\end{array}$ & $\begin{array}{l}0,92 \\
(0,91-0,92)^{*}\end{array}$ & $\begin{array}{l}0,89 \\
(0,89-0,90)^{*}\end{array}$ & $\begin{array}{l}1,02 \\
(1,00-1,04)\end{array}$ \\
\hline Nouvelle-Écosse & $\begin{array}{l}0,97 \\
(0,95-0,99)^{*}\end{array}$ & $\begin{array}{l}0,99 \\
(0,98-1,00)\end{array}$ & $\begin{array}{l}0,89 \\
(0,88-0,90)^{*}\end{array}$ & $\begin{array}{l}0,87 \\
(0,83-0,92)^{*}\end{array}$ & $\begin{array}{l}1,05 \\
(1,03-1,06)^{*}\end{array}$ & $\begin{array}{l}1,03 \\
(1,02-1,03)^{*}\end{array}$ & $\begin{array}{l}0,88 \\
(0,87-0,88)^{*}\end{array}$ & $\begin{array}{l}0,93 \\
(0,90-0,96)^{*}\end{array}$ \\
\hline \multicolumn{9}{|c|}{ Arthrite rhumatoïde (714) ${ }^{\mathrm{a}}$} \\
\hline Colombie-Britannique & $\begin{array}{l}1,09 \\
(0,99-1,19)\end{array}$ & $\begin{array}{l}1,56 \\
(1,49-1,64)^{*}\end{array}$ & $\begin{array}{l}0,93 \\
(0,88-0,98)^{*}\end{array}$ & $\begin{array}{l}1,79 \\
(1,60-2,00)^{*}\end{array}$ & $\begin{array}{l}1,06 \\
(1,00-1,13)^{*}\end{array}$ & $\begin{array}{l}1,42 \\
(1,38-1,46)^{*}\end{array}$ & $\begin{array}{l}1,16 \\
(1,13-1,20)^{*}\end{array}$ & $\begin{array}{l}2,48 \\
(2,33-2,63)^{*}\end{array}$ \\
\hline Nouvelle-Écosse & $\begin{array}{l}1,38 \\
(1,20-1,59)^{*}\end{array}$ & $\begin{array}{l}1,11 \\
(1,03-1,21)^{*}\end{array}$ & $\begin{array}{l}0,89 \\
(0,83-0,95)^{*}\end{array}$ & $\begin{array}{l}0,60 \\
(0,37-0,97)^{*}\end{array}$ & $\begin{array}{l}1,10 \\
(1,00-1,22)\end{array}$ & $\begin{array}{l}1,08 \\
(1,03-1,14)^{*}\end{array}$ & $\begin{array}{l}0,90 \\
(0,87-0,94)^{*}\end{array}$ & $\begin{array}{l}1,00 \\
(0,79-1,25)\end{array}$ \\
\hline Nouvelle-Écosse & $\begin{array}{l}1,14 \\
(1,05-1,23)^{*}\end{array}$ & $\begin{array}{l}0,96 \\
(0,92-1,00)\end{array}$ & $\begin{array}{l}0,84 \\
(0,81-0,87)^{*}\end{array}$ & $\begin{array}{l}1,00 \\
(0,83-1,22)\end{array}$ & $\begin{array}{l}1,15 \\
(1,07-1,23)^{*}\end{array}$ & $\begin{array}{l}1,02 \\
(0,98-1,06)\end{array}$ & $\begin{array}{l}0,94 \\
(0,91-0,97)^{*}\end{array}$ & $\begin{array}{l}0,99 \\
(0,85-1,16)\end{array}$ \\
\hline \multicolumn{9}{|c|}{ Arthrite rhumatoïde et ostéoarthrite combinées $(714-715)^{a}$} \\
\hline Ontario & $\begin{array}{l}1,07 \\
(1,06-1,09)^{*}\end{array}$ & $\begin{array}{l}1,16 \\
(1,15-1,18)^{*}\end{array}$ & $\begin{array}{l}1,05 \\
(1,03-1,07)^{*}\end{array}$ & $\begin{array}{l}1,20 \\
(1,14-1,27)^{*}\end{array}$ & $\begin{array}{l}0,95 \\
(0,94-0,95)^{*}\end{array}$ & $\begin{array}{l}0,97 \\
(0,96-0,99)^{*}\end{array}$ & $\begin{array}{l}0,94 \\
(0,92-0,95)^{*}\end{array}$ & $\begin{array}{l}1,39 \\
(1,33-1,44)^{*}\end{array}$ \\
\hline
\end{tabular}

Sources des données : Base de données sur la facturation des médecins de la Nouvelle-Écosse, 2001-2002; Base de données sur la facturation des médecins de l'Ontario, 2001-2002; Régime des services médicaux de la Colombie-Britannique, 2000-2001.

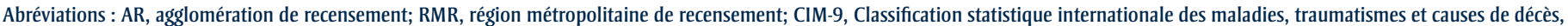
neuvième édition; ZIM, zone d'influence métropolitaine.

a Codes de diagnostic de la CIM-9.

* Statistiquement significatif à $p<0,05$; le groupe de référence est la RMR-AR (risque $=1,00$ ).

TABLEAU A8

Risques relatifs de congé d'hôpital (normalisé selon l'âge) pour maladies de l'appareil locomoteur et du tissu conjonctif, selon le lieu de résidence et le sexe, Ontario, Nouvelle-Écosse et Colombie-Britannique, 2001-2002

\begin{tabular}{llcccc}
\hline Province & Sexe & ZIM forte & ZIM modérée & ZIM faible & \multicolumn{1}{c}{ ZIM nulle } \\
\hline \multirow{2}{*}{ Ontario } & Hommes & $1,31(1,20-1,42)^{*}$ & $1,40(1,33-1,48)^{*}$ & $1,76(1,69-1,84)^{*}$ & $1,69(1,42-2,00)^{*}$ \\
& Femmes & $1,16(1,10-1,22)^{*}$ & $1,40(1,33-1,48)^{*}$ & $1,66(1,55-1,78)^{*}$ & $1,93(1,65-2,26)^{*}$ \\
\hline \multirow{2}{*}{ Nouvelle-Écosse } & Hommes & $1,32(1,05-1,66)^{*}$ & $1,16(1,00-1,34)^{*}$ & $0,98(0,87-1,10)$ & $3,95(2,83-5,52)^{*}$ \\
& Femmes & $1,45(1,14-1,83)^{*}$ & $1,19(1,03-1,39)^{*}$ & $1,06(0,94-1,20)$ & $5,35(3,99-7,18)^{*}$ \\
\hline \multirow{2}{*}{ Colombie-Britannique } & Hommes & $1,10(0,93-1,30)$ & $1,35(1,24-1,49)^{*}$ & $1,54(1,42-1,67)^{*}$ & $1,71(1,38-2,10)^{*}$ \\
& Femmes & $1,13(0,96-1,34)$ & $1,20(1,09-1,32)^{*}$ & $1,81(1,68-1,96)^{*}$ & $2,46(2,04-2,97)^{*}$ \\
\hline
\end{tabular}

Source des données : Base de données sur la morbidité hospitalière, 2001-2002, Institut canadien d'information sur la santé.

Abréviations : AR, aggglomération de recensement; RMR, région métropolitaine de recensement; ZIM, zone d'influence métropolitaine.

* Statistiquement significatif à $p<0,05$; le groupe de référence est la RMR-AR (risque $=1,00$ ). 
TABLEAU A9

Risques relatifs de consultation chez le médecin (normalisée selon l’âge) pour traumatisme ou intoxication, selon le lieu de résidence et le sexe, Ontario et Nouvelle-Écosse, 2001-2002

\begin{tabular}{|c|c|c|c|c|c|c|c|c|}
\hline & \multicolumn{4}{|c|}{ Hommes } & \multicolumn{4}{|c|}{ Femmes } \\
\hline & ZIM forte & $\begin{array}{c}\text { ZIM } \\
\text { modérée }\end{array}$ & ZIM faible & ZIM nulle & ZIM forte & $\begin{array}{c}\text { ZIM } \\
\text { modérée }\end{array}$ & ZIM faible & ZIM nulle \\
\hline \multicolumn{9}{|c|}{ Traumatisme et intoxication $(802-894,977-998 \text { et } 959)^{a}$} \\
\hline Ontario & $\begin{array}{l}0,98 \\
(0,98-0,99)^{*}\end{array}$ & $\begin{array}{l}1,10 \\
(1,09-1,10)^{*}\end{array}$ & $\begin{array}{l}1,15 \\
(1,14-1,16)^{*}\end{array}$ & $\begin{array}{l}1,17 \\
(1,15-1,20)^{*}\end{array}$ & $\begin{array}{l}0,94 \\
(0,94-0,95)^{*}\end{array}$ & $\begin{array}{l}1,01 \\
(1,00-1,02)^{*}\end{array}$ & $\begin{array}{l}1,06 \\
(1,05-1,07)^{*}\end{array}$ & $\begin{array}{l}1,23 \\
(1,20-1,26)^{*}\end{array}$ \\
\hline Nouvelle-Écosse & $\begin{array}{l}1,19 \\
(1,16-1,23)^{*}\end{array}$ & $\begin{array}{l}1,18 \\
(1,16-1,19)^{*}\end{array}$ & $\begin{array}{l}1,08 \\
(1,06-1,09)^{*}\end{array}$ & $\begin{array}{l}1,21 \\
(1,14-1,28)^{*}\end{array}$ & $\begin{array}{l}1,22 \\
(1,19-1,25)^{*}\end{array}$ & $\begin{array}{l}1,11 \\
(1,09-1,12)^{*}\end{array}$ & $\begin{array}{l}0,98 \\
(0,97-0,99)^{*}\end{array}$ & $\begin{array}{l}0,96 \\
(0,90-1,03)\end{array}$ \\
\hline
\end{tabular}

Sources des données : Base de données sur la facturation des médecins de la Nouvelle-Écosse, 2001-2002; Base de données sur la facturation des médecins de l’Ontario, $2001-2002$.

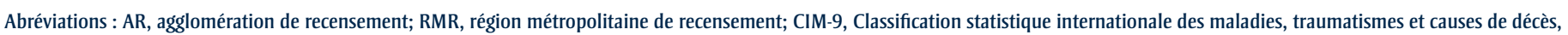
neuvième édition; ZIM, zone d'influence métropolitaine.

a Codes de diagnostic de la CIM-9.

Statistiquement significatif à $p<0,05$; le groupe de référence est la RMR-AR (risque $=1,00$ ).

\section{TABLEAU A10}

Risques relatifs de congé d'hôpital (normalisé selon l'âge) pour traumatisme ou intoxication, selon le lieu de résidence et le sexe, Ontario, Nouvelle-Écosse et Colombie-Britannique, 2001-2002

\begin{tabular}{|c|c|c|c|c|c|}
\hline Province & Sexe & ZIM forte & ZIM modérée & ZIM faible & ZIM nulle \\
\hline \multirow{2}{*}{ Ontario } & Hommes & $1,31(1,24-1,39)^{*}$ & $1,52(1,46-1,58)^{*}$ & $1,94(1,88-2,00)^{*}$ & $2,46(2,22-2,73)^{*}$ \\
\hline & Femmes & $1,25(1,20-1,30)^{*}$ & $1,48(1,41-1,54)^{*}$ & $1,90(1,80-2,00)^{*}$ & $3,19(2,88-3,53)^{*}$ \\
\hline \multirow{2}{*}{ Nouvelle-Écosse } & Hommes & $1,15(0,96-1,39)$ & $1,30(1,17-1,44)^{*}$ & $1,09(1,00-1,19)^{*}$ & $2,06(1,46-2,91)^{*}$ \\
\hline & Femmes & $1,32(1,08-1,62)^{*}$ & $1,29(1,14-1,45)^{*}$ & $0,97(0,88-1,08)$ & $2,01(1,36-2,98)^{*}$ \\
\hline \multirow{2}{*}{ Colombie-Britannique } & Hommes & $0,96(0,86-1,07)$ & $1,34(1,27-1,42)^{*}$ & $1,96(1,88-2,06)^{*}$ & $1,90(1,68-2,15)^{*}$ \\
\hline & Femmes & $1,01(0,89-1,14)$ & $1,21(1,13-1,30)^{*}$ & $1,93(1,83-2,04)^{*}$ & $2,23(1,94-2,56)^{*}$ \\
\hline
\end{tabular}

Source des données : Base de données sur la morbidité hospitalière, 2001-2002, Institut canadien d'information sur la santé.

* Statistiquement significatif à $p<0,05$; le groupe de référence est la RMR-AR (risque $=1,00$ ).

Abréviations : AR, agglomération de recensement; RMR, région métropolitaine de recensement; ZIM, zone d'influence métropolitaine.

TABLEAU A11

Risques relatifs de consultation chez le médecin (normalisée selon l'âge) pour troubles mentaux, selon le lieu de résidence et le sexe, Ontario et Nouvelle-Écosse, 2001-2002 et Colombie-Britannique, 2000-2001

\begin{tabular}{|c|c|c|c|c|c|c|c|c|}
\hline & \multicolumn{4}{|c|}{ Hommes } & \multicolumn{4}{|c|}{ Femmes } \\
\hline & ZIM forte & $\begin{array}{c}\text { ZIM } \\
\text { modérée }\end{array}$ & ZIM faible & ZIM nulle & ZIM forte & $\begin{array}{c}\text { ZIM } \\
\text { modérée }\end{array}$ & ZIM faible & ZIM nulle \\
\hline \multicolumn{9}{|c|}{ Troubles mentaux (290-319)a } \\
\hline Ontario & $\begin{array}{l}0,64 \\
(0,63-0,64)^{*}\end{array}$ & $\begin{array}{l}0,65 \\
(0,65-0,66)^{*}\end{array}$ & $\begin{array}{l}0,60 \\
(0,59-0,60)^{*}\end{array}$ & $\begin{array}{l}0,54 \\
(0,53-0,56)^{*}\end{array}$ & $\begin{array}{l}0,74 \\
(0,74-0,74)^{*}\end{array}$ & $\begin{array}{l}0,70 \\
(0,70-0,71)^{*}\end{array}$ & $\begin{array}{l}0.69 \\
(0,69-0,70)^{*}\end{array}$ & $\begin{array}{l}0,50 \\
(0,49-0,51)^{*}\end{array}$ \\
\hline Nouvelle-Écosse & $\begin{array}{l}0,75 \\
(0,73-0,78)^{*}\end{array}$ & $\begin{array}{l}0,84 \\
(0,83-0,85)^{*}\end{array}$ & $\begin{array}{l}0,70 \\
(0,69-0,71)^{*}\end{array}$ & $\begin{array}{l}0,88 \\
(0,82-0,94)^{*}\end{array}$ & $\begin{array}{l}0,94 \\
(0,92-0,95)^{*}\end{array}$ & $\begin{array}{l}0,89 \\
(0,88-0,90)^{*}\end{array}$ & $\begin{array}{l}0,73 \\
(0,72-0,74)^{*}\end{array}$ & $\begin{array}{l}0,80 \\
(0,76-0,84)^{*}\end{array}$ \\
\hline \multicolumn{9}{|c|}{ Dépression $(296.2,296.3,300.4,311)^{a}$} \\
\hline Colombie-Britannique & $\begin{array}{l}0,49 \\
(0,48-0,51)^{*}\end{array}$ & $\begin{array}{l}0,78 \\
(0,77-0,79)^{*}\end{array}$ & $\begin{array}{l}0,50 \\
(0,49-0,51)^{*}\end{array}$ & $\begin{array}{l}0,54 \\
(0,51-0,56)^{*}\end{array}$ & $\begin{array}{l}0,59 \\
(0,58-0,60)^{*}\end{array}$ & $\begin{array}{l}0,84 \\
(0,83-0,85)^{*}\end{array}$ & $\begin{array}{l}0,59 \\
(0,58-0,59)^{*}\end{array}$ & $\begin{array}{l}0,64 \\
(0,62-0,66)^{*}\end{array}$ \\
\hline Nouvelle-Écosse & $\begin{array}{l}0,80 \\
(0,75-0,86)^{*}\end{array}$ & $\begin{array}{l}0,72 \\
(0,70-0,75)^{*}\end{array}$ & $\begin{array}{l}0,69 \\
(0,67-0,71)^{*}\end{array}$ & $\begin{array}{l}0,69 \\
(0,59-0,82)^{*}\end{array}$ & $\begin{array}{l}0,96 \\
(0,92-1,00)\end{array}$ & $\begin{array}{l}0,74 \\
(0,73-0,76)^{*}\end{array}$ & $\begin{array}{l}0,71 \\
(0,70-0,72)^{*}\end{array}$ & $\begin{array}{l}0,75 \\
(0,68-0,82)^{*}\end{array}$ \\
\hline
\end{tabular}

Sources des données : Base de données sur la facturation des médecins de la Nouvelle-Écosse, 2001-2002; Base de données sur la facturation des médecins de l'Ontario, 2001-2002; Régime des services médicaux de la Colombie-Britannique, 2000-2001.

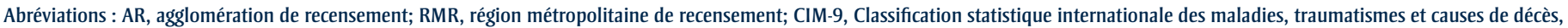
neuvième édition; ZIM, zone d'influence métropolitaine.

a Codes de diagnostic de la CIM-9.

Statistiquement significatif à $p<0,05$; le groupe de référence est la RMR-AR (risque $=1,00$ ). 
TABLEAU A12

Risques relatifs de congé d'hôpital (normalisé selon l'âge) pour troubles mentaux, selon le lieu de résidence et le sexe, Ontario, Nouvelle-Écosse et Colombie-Britannique, 2001-2002

\begin{tabular}{|c|c|c|c|c|c|}
\hline Province & Sex & ZIM forte & ZIM modérée & ZIM faible & ZIM nulle \\
\hline \multirow{2}{*}{ Ontario } & Hommes & $0,82(0,75-0,90)^{*}$ & $1,54(1,46-1,61)^{*}$ & $1,99(1,92-2,07)^{*}$ & $2,79(2,47-3,15)^{*}$ \\
\hline & Femmes & $0,82(0,78-0,87)^{*}$ & $1,54(1,46-1,61)^{*}$ & $1,99(1,88-2,12)^{*}$ & $2,79(2,46-3,16)^{*}$ \\
\hline \multirow{2}{*}{ Nouvelle-Écosse } & Hommes & $0,84(0,65-1,11)$ & $0,81(0,68-0,96)^{*}$ & $1,37(1,23-1,53)^{*}$ & $3,69(2,58-5,29)^{*}$ \\
\hline & Femmes & $0,83(0,62-1,11)$ & $0,83(0,70-0,98)^{*}$ & $1,24(1,11-1,38)^{*}$ & $7,56(5,97-9,58)^{*}$ \\
\hline \multirow{2}{*}{ Colombie-Britannique } & Hommes & $0,62(0,52-0,73)^{*}$ & $1,21(1,12-1,30)^{*}$ & $1,52(1,42-1,62)^{*}$ & $1,65(1,40-1,94)^{*}$ \\
\hline & Femmes & $0,63(0,53-0,74)^{*}$ & $0,98(0,91-1,07)$ & $1,69(1,60-1,80)^{*}$ & $1,76(1,49-2,08)^{*}$ \\
\hline
\end{tabular}

Source des données : Base de données sur la morbidité hospitalière, 2001-2002, Institut canadien d'information sur la santé.

Abréviations : AR, agglomération de recensement; RMR, région métropolitaine de recensement; ZIM, zone d'influence métropolitaine.

* Statistiquement significatif à $p<0,05$; le groupe de référence est la RMR-AR (risque $=1,00$ ).

TABLEAU A13

Risques relatifs de consultation chez le médecin (normalisée selon l'âge) pour maladies du système nerveux ou des organes sensoriels, selon le lieu de résidence et le sexe, Ontario et Nouvelle-Écosse, 2001-2002 et Colombie-Britannique, 2000-2001

\begin{tabular}{|c|c|c|c|c|c|c|c|c|}
\hline & \multicolumn{4}{|c|}{ Hommes } & \multicolumn{4}{|c|}{ Femmes } \\
\hline & ZIM forte & $\begin{array}{c}\text { ZIM } \\
\text { modérée }\end{array}$ & ZIM faible & ZIM nulle & ZIM forte & $\begin{array}{c}\text { ZIM } \\
\text { modérée }\end{array}$ & ZIM faible & ZIM nulle \\
\hline Ontario & $\begin{array}{l}0,83 \\
(0,82-0,83)^{*}\end{array}$ & $\begin{array}{l}0,83 \\
(0,82-0,83)^{*}\end{array}$ & $\begin{array}{l}0,78 \\
(0,78-0,79)^{*}\end{array}$ & $\begin{array}{l}0,78 \\
(0,76-0,80)^{*}\end{array}$ & $\begin{array}{l}0,87 \\
(0,86-0,87)^{*}\end{array}$ & $\begin{array}{l}0,84 \\
(0,84-0,85)^{*}\end{array}$ & $\begin{array}{l}0,84 \\
(0,83-0,84)^{*}\end{array}$ & $\begin{array}{l}0,87 \\
(0,85-0,89)^{*}\end{array}$ \\
\hline Nouvelle-Écosse & $\begin{array}{l}0,97 \\
(0,95-0,99)^{*}\end{array}$ & $\begin{array}{l}1,01 \\
(1,00-1,03)^{*}\end{array}$ & $\begin{array}{l}0,97 \\
(0,96-0,97)^{*}\end{array}$ & $\begin{array}{l}0,85 \\
(0,80-0,89)^{*}\end{array}$ & $\begin{array}{l}0,99 \\
(0,97-1,01)\end{array}$ & $\begin{array}{l}1,06 \\
(1,05-1,06)^{*}\end{array}$ & $\begin{array}{l}0,97 \\
(0,97-0,98)^{*}\end{array}$ & $\begin{array}{l}0,92 \\
(0,88-0,95)^{*}\end{array}$ \\
\hline \multicolumn{9}{|c|}{ Maladie d'Alzheimer/troubles démentiels (331) ${ }^{\mathrm{a}}$} \\
\hline Colombie-Britannique & $\begin{array}{l}0,47 \\
(0,34-0,64)^{*}\end{array}$ & $\begin{array}{l}1,43 \\
(1,27-1,60)^{*}\end{array}$ & $\begin{array}{l}0,77 \\
(0,94-0,88)^{*}\end{array}$ & $\begin{array}{l}0,73 \\
(0,50-1,08)\end{array}$ & $\begin{array}{l}1,18 \\
(1,02-1,37)^{*}\end{array}$ & $\begin{array}{l}1,73 \\
(1,61-1,87)^{*}\end{array}$ & $\begin{array}{l}0,82 \\
(0,74-0,91)^{*}\end{array}$ & $\begin{array}{l}0,62 \\
(0,45-0,86)^{*}\end{array}$ \\
\hline Nouvelle-Écosse & $\begin{array}{l}0,83 \\
(0,54-1,29)\end{array}$ & $\begin{array}{l}1,09 \\
(0,90-1,33)\end{array}$ & $\begin{array}{l}0,74 \\
(0,62-0,88)^{*}\end{array}$ & $\begin{array}{l}2,11 \\
(1,13-3,95)^{*}\end{array}$ & $\begin{array}{l}1,08 \\
(0,73-1,58)\end{array}$ & $\begin{array}{l}1,22 \\
(1,02-1,46)^{*}\end{array}$ & $\begin{array}{l}0,84 \\
(0,72-0,98)^{*}\end{array}$ & $\begin{array}{l}1,66 \\
(0,86-3,18)\end{array}$ \\
\hline
\end{tabular}

Sources des données : Base de données sur la facturation des médecins de la Nouvelle-Écosse, 2001-2002; Base de données sur la facturation des médecins de l’Ontario, 2001-2002; Régime des services médicaux de la Colombie-Britannique, 2000-2001.

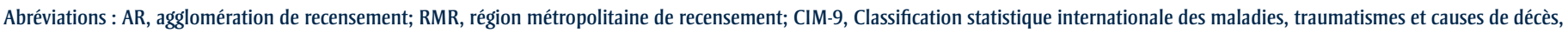
neuvième édition; ZIM, zone d'influence métropolitaine.

a Codes de diagnostic de la CIM-9.

* Statistiquement significatif à $p<0,05$; le groupe de référence est la RMR-AR (risque $=1,00$ ). 
TABLEAU A14

Risques relatifs de congé d'hôpital (normalisé selon l'âge) pour maladies du système nerveux ou des organes sensoriels, selon le lieu de résidence et le sexe, Ontario, Nouvelle-Écosse et Colombie-Britannique, 2001-2002

\begin{tabular}{|c|c|c|c|c|c|}
\hline Province & Sex & ZIM forte & ZIM modérée & ZIM faible & ZIM nulle \\
\hline \multirow[t]{2}{*}{ Ontario } & Hommes & $1,03(0,89-1,19)$ & $1,44(1,32-1,57)^{*}$ & $1,72(1,61-1,84)^{*}$ & $2,00(1,56-2,56)^{*}$ \\
\hline & Femmes & $1,16(1,06-1,26)^{*}$ & $1,63(1,49-1,77)^{*}$ & $2,07(1,86-2,31)^{*}$ & $1,68(1,25-2,25)^{*}$ \\
\hline \multirow[t]{2}{*}{ Nouvelle-Écosse } & Hommes & $1,61(1,23-2,11)^{*}$ & $1,21(1,01-1,46)^{*}$ & $1,07(0,92-1,24)$ & $7,40(5,38-10,2)^{*}$ \\
\hline & Femmes & $1,48(1,09-2,00)^{*}$ & $1,23(1,02-1,49)^{*}$ & $1,18(1,02-1,37)^{*}$ & $4,61(3,05-6,97)^{*}$ \\
\hline \multirow[t]{2}{*}{ Colombie-Britannique } & Hommes & $0,78(0,61-0,99)^{*}$ & $1,03(0,91-1,17)$ & $1,49(1,34-1,65)^{*}$ & $1,54(1,17-2,02)^{*}$ \\
\hline & Femmes & $0,85(0,66-1,11)$ & $1,01(0,87-1,16)$ & $1,66(1,49-1,85)^{*}$ & $2,80(2,21-3,56)^{*}$ \\
\hline
\end{tabular}

Source des données : Base de données sur la morbidité hospitalière, 2001-2002, Institut canadien d'information sur la santé.

Abréviations : AR, aggglomération de recensement; RMR, région métropolitaine de recensement; ZIM, zone d'influence métropolitaine.

* Statistiquement significatif à $p<0,05$; le groupe de référence est la RMR-AR (risque $=1,00$ ).

TABLEAU A15

Risques relatifs de consultation chez le médecin (normalisée selon l'âge) pour diabète, selon le lieu de résidence et le sexe, Ontario et Nouvelle-Écosse, 2001-2002, et Colombie-Britannique, 2000-2001

\begin{tabular}{|c|c|c|c|c|c|c|c|c|}
\hline & \multicolumn{4}{|c|}{ Hommes } & \multicolumn{4}{|c|}{ Femmes } \\
\hline & ZIM forte & $\begin{array}{c}\text { ZIM } \\
\text { modérée }\end{array}$ & ZIM faible & ZIM nulle & ZIM forte & $\begin{array}{c}\text { ZIM } \\
\text { modérée }\end{array}$ & ZIM faible & ZIM nulle \\
\hline \multicolumn{9}{|l|}{ Diabète $(250)^{\mathrm{a}}$} \\
\hline Colombie-Britannique & $\begin{array}{l}0,63 \\
(0,60-0,65)^{*}\end{array}$ & $\begin{array}{l}0,74 \\
(0,73-0,76)^{*}\end{array}$ & $\begin{array}{l}0,72 \\
(0,70-0,73)^{*}\end{array}$ & $\begin{array}{l}1,18 \\
(1,14-1,22)^{*}\end{array}$ & $\begin{array}{l}0,73 \\
(0,71-0,76)^{*}\end{array}$ & $\begin{array}{l}0,76 \\
(0,75-0,78)^{*}\end{array}$ & $\begin{array}{l}0,84 \\
(0,82-0,85)^{*}\end{array}$ & $\begin{array}{l}1,35 \\
(1,30-1,41)^{*}\end{array}$ \\
\hline Ontario & $\begin{array}{l}0,86 \\
(0,86-0,87)^{*}\end{array}$ & $\begin{array}{l}0,86 \\
(0,85-0,87)^{*}\end{array}$ & $\begin{array}{l}0,93 \\
(0,92-0,94)^{*}\end{array}$ & $\begin{array}{l}1,57 \\
(1,52-1,62)^{*}\end{array}$ & $\begin{array}{l}0,93 \\
(0,92-0,94)^{*}\end{array}$ & $\begin{array}{l}1,00 \\
(0,99-1,01)\end{array}$ & $\begin{array}{l}0,98 \\
(0,97-1,00)^{*}\end{array}$ & $\begin{array}{l}2,11 \\
(2,05-2,17)^{*}\end{array}$ \\
\hline Nouvelle-Écosse & $\begin{array}{l}1,31 \\
(1,27-1,36)^{*}\end{array}$ & $\begin{array}{l}0,91 \\
(0,89-0,93)^{*}\end{array}$ & $\begin{array}{l}0,89 \\
(0,88-0,91)^{*}\end{array}$ & $\begin{array}{l}1,21 \\
(1,11-1,32)^{*}\end{array}$ & $\begin{array}{l}1,35 \\
(1,29-1,41)^{*}\end{array}$ & $\begin{array}{l}1,08 \\
(1,05-1,11)^{*}\end{array}$ & $\begin{array}{l}1,01 \\
(0,99-1,03)\end{array}$ & $\begin{array}{l}1,66 \\
(1,53-1,79)^{*}\end{array}$ \\
\hline
\end{tabular}

Sources des données : Base de données sur la facturation des médecins de la Nouvelle-Écosse, 2001-2002; Base de données sur la facturation des médecins de l’Ontario, 2001-2002; Régime des services médicaux de la Colombie-Britannique, 2000-2001.

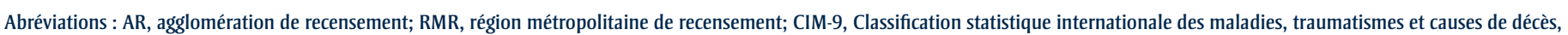
neuvième édition; ZIM, zone d'influence métropolitaine.

a Code de diagnostic de la CIM-9.

* Statistiquement significatif à $p<0,05$; le groupe de référence est la RMR-AR (risque $=1,00$ ). 


\section{MCBC : Information à l'intention des auteurs}

Maladies chroniques et blessures au Canada (MCBC) est une revue scientifique trimestrielle dont les articles de fond sont soumis à un examen par les pairs. La revue s'intéresse particulièrement à la prévention et la lutte contre les maladies non transmissibles et les traumatismes au Canada. Ce champ d'intérêt peut englober les recherches effectuées dans des domaines tels que l'épidémiologie, la santé publique ou communautaire, la biostatistique, les sciences du comportement, et l'économie ou les services de la santé. La revue s'efforce de stimuler la communication au sujet des maladies chroniques et des traumatismes entre les professionnels en santé publique, les épidémiologistes et chercheurs, et les personnes qui participent à la planification de politiques en matière de santé et à l'éducation à la santé. Le choix des articles repose sur les critères suivants : valeur scientifique, pertinence sur le plan de la santé publique, clarté, concision et exactitude technique. Bien que MCBC soit une publication de l'Agence de la santé publique du Canada, nous acceptons des articles d'auteurs des secteurs public et privé. Les auteurs demeurent responsables du contenu de leurs articles, et les opinions exprimées ne sont pas forcément celles du Comité de rédaction de MCBC ni celles de l'Agence de la santé publique du Canada.

\section{Types d'articles}

Article de fond (soumis à un examen par les pairs) : Le corps du texte ne doit pas comporter plus de 4000 mots (sans compter le résumé, les tableaux, les figures et la liste de références). Il peut s'agir de travaux de recherche originaux, de rapports de surveillance, de méta-analyses, ou de documents de méthodologie.

Rapport de la situation : Description des programmes, des études ou des systèmes d'information ayant trait à la santé publique canadienne (maximum de 3000 mots). Sans résumé.

Rapport de conférence/d'atelier : Résumés d'événements d'envergure récents ayant des liens avec la santé publique nationale (ne doit pas dépasser 1200 mots). Sans résumé.

Forum pancanadien : Les auteurs peuvent partager de l'information portant sur les résultats de surveillance, des programmes en cours d'élaboration ou des initiatives liées à la politique en matière de la santé publique, tant au niveau national que régional (maximum de 3000 mots). Sans résumé.
Lettre au rédacteur : L'on envisage la publication des observations au sujet d'articles récemment parus dans MCBC (maximum 500 mots). Sans résumé.

Recension de livres/logiciels : La rédaction les sollicitent d'habitude (500-1 300 mots), mais les demandes à réviser sont appréciées. Sans résumé.

\section{Présentation des manuscrits}

Les manuscrits doivent être adressés à la gestionnaire de la rédaction, Maladies chroniques et blessures au Canada, Agence de santé publique du Canada, 785, avenue Carling, Indice de l'adresse : 6805B, Ottawa (Ontario) K1A 0K9, courriel : cdic-mcbc@phac-aspc.gc.ca.

Maladies chroniques et blessures au Canada suit en général (à l'exception de la section sur les illustrations) les "Exigences uniformes pour les manuscrits présentés aux revues biomédicales", approuvées par le Comité international des rédacteurs de revues médicales. Pour plus de précisions, les auteurs sont priés de consulter ce document avant de soumettre un manuscrit à MCBC (voir < www.icmje.org > ).

\section{Liste de vérification pour la présentation des manuscrits}

Lettre d'accompagnement : Signée par tous les auteurs, elle doit indiquer que tous les auteurs ont pris connaissance de la version finale du document, l'ont approuvée et ont satisfait aux critères applicables à la paternité de l'œuvre figurant dans les Exigences uniformes et elle doit également comporter un énoncé en bonne et due forme faisant état de toute publication (ou soumission pour publication) antérieure ou supplémentaire.

Première page titre : Titre concis avec les noms complets de tous les auteurs avec leur affiliation, le nom de l'auteur chargé de la correspondance, son adresse postale et son adresse de courrier électronique, son numéro de téléphone et son numéro de télécopieur. Le dénombrement des mots du texte et du résumé se font séparément.

Deuxième page titre : Titre seulement et début de la numérotation des pages.

Résumé : Non structuré (un paragraphe, pas de titres), moins de 175 mots (maximum de 100 s'il s'agit d'un article court) suivi de trois à huit mots clés, de préférence choisis parmi les mots clés MeSH (Medical Subject Headings) de l'Index Medicus.

Remerciements : Mentionnez toute aide matérielle ou financière dans les remerciements. Si des remerciements sont faits à une personne pour une contribution scientifique majeure, les auteurs doivent mentionner dans la lettre d'accompagnement qu'ils en ont obtenu la permission écrite.

Références : Les références devraient être conformes au " code de style de Vancouver " (consultez un numéro récent de $\mathrm{MCBC}$ à titre d'exemple), numérotées à la suite, dans l'ordre où elles apparaissent pour la première fois dans le texte, les tableaux ou les figures (avec des chiffres en exposants ou entre parenthèses); mentionnez jusqu'à six auteurs (les trois premiers et " et collab. " s'il y en a plus) et enlevez toute fonction automatique de numérotation des références employée dans le traitement de texte. Toute observation/donnée inédite ou communication personnelle citée en référence (à éviter) devrait être intégrée au texte, entre parenthèses. Il incombe aux auteurs d'obtenir l'autorisation requise et de veiller à l'exactitude de leurs références.

Tableaux et figures : Seulement les graphiques vectorisés sont acceptables. Mettez les tableaux et les figures sur des pages distinctes et dans un (des) fichier(s) différent(s) de celui du texte (ne les intégrez pas dans le corps du texte). Ils doivent être aussi explicites et succincts que possible et ne pas être trop nombreux. Numérotez-les dans l'ordre de leur apparition dans le texte, et mettez les renseignements complémentaires comme notes au bas du tableau, identifiées par des lettres minuscules en exposants, selon l'ordre alphabétique. Présentez les figures sous forme de graphiques, diagrammes ou modèles (pas d’images), précisez le logiciel utilisé et fournissez les titres et les notes de bas de page sur une page séparée.

Nombre de copies : Par courrier - une version complète avec tableaux et figures; une copie de tout matériel connexe, et une copie du manuscrit sur disquette ou disque compact. Par courriel - au cdic-mcbc@phac-aspc.gc.ca et lettre d'accompagnement par télécopieur ou courrier à l'adresse indiquée à la couverture avant intérieure. 
